Supporting Information

\title{
Effects of Halogenated WNA Derivatives on Sequence Dependency for Expansion of Recognition Sequences in the Non-Natural Type Triplexes
}

Yosuke Taniguchi, Ayako Nakamura, Yusuke Senko, Fumi Nagatsugi† and Shigeki Sasaki*, $\dagger$

Graduate School of Pharmaceutical Sciences, Kyushu University, 3-1-1 Maidashi, Higashi-ku, Fukuoka 812-8582, Japan, †CREST, Japan Science and Technology Agency, Kawaguchi Center Building, 4-1-8 Honcho, Kawaguchi, Saitama 332-0012, Japan

TEL\&FAX +81-92-642-6615

E-mail: sasaki@phar.kyushu-u.ac.jp

Contents

Experimental General $\quad$ S2

Glycosidation $\quad$ S2

Deprotection $\quad$ S5

Synthesis of the $\beta$-cyanoethylphosphoramidite precursors S8

$\begin{array}{ll}\text { Scheme } 2 & \text { S11 }\end{array}$

COSY, NOESY, ${ }^{1} \mathrm{H}$ and ${ }^{13} \mathrm{C}$ NMR spectra $\quad$ S27 
General. ${ }^{1} \mathrm{H}$ and ${ }^{13} \mathrm{C}$ NMR were recorded at $400 / 100 \mathrm{MHz}$ or $500 / 125 \mathrm{MHz}$. Chemical shifts $(\delta)$ are given in parts per million (ppm) using chloroform-d $\left(\mathrm{CHCl}_{3} \delta_{\mathrm{H}}\right.$ 7.26, $\mathrm{CDCl}_{3} \delta_{\mathrm{C}} 77.0$ ), or deuterium oxide ( $\left.\mathrm{DOH} \delta_{\mathrm{H}} 4.75\right)$ as internal references. ${ }^{31} \mathrm{P}$ NMR spectrum was recorded at $161.9 \mathrm{MHz}$ using $10 \% \mathrm{H}_{3} \mathrm{PO}_{4}$ in $\mathrm{D}_{2} \mathrm{O}$ as an external standard. IR spectra were recorded as film or $\mathrm{KBr}$ tablets. High-resolution mass spectrometry (HRMS) measurements were performed on a mass spectrometer equipped with an external electrospray ion source using bradykinin, neurotensin, and angiotensin as an internal standard. MALDI-TOF MS spectrometry measurements were performed in negative mode by using 3-hydroxy-2-picolinic acid and diammonium hydrogen citrate as the matrix. Column chromatography was carried out using 63-210 $\mu \mathrm{m}$ silica gel. HPLC was performed on ODS columns monitoring at $254 \mathrm{~nm}$.

\section{Glycosidation}

(1'S,3'R,4'R,5'R,7'S)-1-\{4'-Acetoxy-3'-(tert-butyldiphenylsilyoxymethyl)-1'-phenyl -2',6'-dioxabicyclo[3.3.0]oct-7'-yl\}-5-fluorouracil (WNA- $\beta$ FU, 17a). Reagents: 5-fluorouracil (120 mg, $0.871 \mathrm{mmol})$, BSA (424 $\mu \mathrm{L}, 1.74 \mathrm{mmol}), 15$ (400 mg, 0.697 mmol), TMSOTf (189 $\mu \mathrm{L}, 1.05 \mathrm{mmol}) . \quad$ A colorless foam (264 mg, 59\%). $\quad{ }^{1} \mathrm{H}$ NMR 
(400 MHz, CDCl 3 /TMS) $\delta 8.82$ (bs, 1H), 7.66 (d, 2H, $J=7.7 \mathrm{~Hz}$ ), 7.61 (d, 2H, $J=7.9$ Hz), 7.54 (d, 2H, $J=7.7 \mathrm{~Hz}), 7.50$ (d, 1H, $J=5.8 \mathrm{~Hz}$ ), 7.44-7.29 (m, 9H), 6.30 (dd, 1H, $J=$ 8.6, $5.6 \mathrm{~Hz}$ ), 5.12 (d, 1H, $J=3.9 \mathrm{~Hz}$ ), 5.07 (dd, 1H, $J=9.0,3.9 \mathrm{~Hz}$ ), 4.25 (ddd, $1 \mathrm{H}$, $J=9.0,3.6,3.0 \mathrm{~Hz}$ ), 4.00 (dd, $1 \mathrm{H}, J=11.8,3.0 \mathrm{~Hz}$ ), 3.74 (dd, $1 \mathrm{H}, J=11.8,3.6 \mathrm{~Hz}$ ), 2.97 (dd, 1H, $J=14.2,5.6 \mathrm{~Hz}$ ), 2.47 (dd, 1H, $J=14.2$, $8.6 \mathrm{~Hz}$ ), 2.05 (s, 3H), 1.01 (s,

9H). $\quad{ }^{13} \mathrm{C}$ NMR $\left(125 \mathrm{MHz}, \mathrm{CDCl}_{3}\right) \delta 170.2,156.4,148.5,141.9,140.0,139.5,135.9$, 135.8, 133.1, 130.0, 128.9, 128.3, 128.0, 127.9, 125.4, 92.5, 88.5, 86.8, 81.0, 73.3, 62.8, 48.8, 27.0, 20.9, 19.4. FTIR (film) 3184, 3066, 2934, 2856, 1705, 1663, 1471, 1361 $\mathrm{cm}^{-1}$. HRMS (ESIMS) $\mathrm{m} / \mathrm{z}$ calcd for $\mathrm{C}_{35} \mathrm{H}_{37} \mathrm{~N}_{2} \mathrm{O}_{7} \mathrm{SiFNa}(\mathrm{M}+\mathrm{Na})^{+} 667.2246$, found 667.2285.

(1'S,3'R,4'R,5'R,7'S)-N $N^{4}$-Benzoyl-1-\{4'-acetoxy-3'-(tert-butyldiphenylsilyoxymethyl )-1'-phenyl-2',6'-dioxabicyclo[3.3.0]oct-7'-yl\}-5-bromocytosine (WNA- $\beta B r C, 18 a)$. Reagents: $N^{4}$-benzoyl-5-bromocytosine (256 mg, $0.871 \mathrm{mmol}$ ), BSA (424 $\mu \mathrm{L}, 1.74$ mmol), 15 (400 mg, $0.697 \mathrm{mmol})$, TMSOTf (189 $\mu \mathrm{L}, 1.05)$. A colorless foam (296 mg, 52\%). $\quad{ }^{1} \mathrm{H}$ NMR (400 MHz, $\left.\mathrm{CDCl}_{3} / \mathrm{TMS}\right) \delta 13.26$ (bs, $\left.1 \mathrm{H}\right), 8.36$ (d, 2H, $J=7.3$ Hz), 7.88 (s, 1H), 7.67 (d, 2H, $J=7.3 \mathrm{~Hz}$ ), 7.62 (d, 2H, $J=7.3 \mathrm{~Hz}), 7.57-7.54$ (m, 3H), 
7.48-7.30 (m, 11H), 6.30 (dd, 1H, $J=8.3,5.5 \mathrm{~Hz}$ ), 5.18 (d, 1H, $J=4.0 \mathrm{~Hz}$ ), 5.10 (dd, $1 \mathrm{H}, J=4.0,9.1 \mathrm{~Hz}$ ), 4.26 (ddd, 1H, $J=9.1,3.4,3.0 \mathrm{~Hz}$ ), 4.01 (dd, $1 \mathrm{H}, J=11.7,3.0 \mathrm{~Hz}$ ), 3.75 (dd, 1H, $J=11.7,3.4 \mathrm{~Hz}$ ), 3.04 (dd, $1 \mathrm{H}, J=14.2,5.5 \mathrm{~Hz}$ ), 2.53 (dd, $1 \mathrm{H}, J=14.2$, 8.3 Hz), 2.07 (s, 3H), 1.02 (s, 9H). $\left.{ }^{13} \mathrm{C} \mathrm{NMR} \mathrm{(100} \mathrm{MHz,} \mathrm{CDCl}_{3}\right) \delta 170.1,139.9,139.2$, 135.6, 135.6, 133.0, 132.9, 130.2, 129.8, 128.7, 128.3, 127.7, 125.2, 92.3, 89.1, 86.8, 80.7, 73.0, 62.5, 48.8, 26.7, 20.7, 19.2. FTIR (film) 3072, 2930, 2858, 1713, 1634, 1574, 1246, $1113 \mathrm{~cm}^{-1}$. HRMS (ESIMS) m/z calcd for $\mathrm{C}_{42} \mathrm{H}_{43} \mathrm{~N}_{3} \mathrm{O}_{7} \mathrm{SiBr}(\mathrm{M}+\mathrm{H})^{+}$ 808.2048, 810.2038, found 808.1198, 810.2080.

$\left(1^{\prime} S, 3^{\prime} R, 4^{\prime} R, 5^{\prime} R, 7^{\prime} S\right)-N^{4}$-Benzoyl-1-\{4'-Acetoxy-3'-(tert-butyldiphenylsilyoxymethy l)-1'-phenyl-2',6'-dioxabicyclo[3.3.0]oct-7'-yl\}-5-fluorocytosine (WNA- $\beta$ FC, 19a). Reagents: $N^{4}$-benzoyl-5-fluorocytosine (203 mg, $0.871 \mathrm{mmol}$ ), BSA (424 $\mu \mathrm{L}, 1.74$ mmol), 15 (400 mg, $0.697 \mathrm{mmol})$, TMSOTf (189 $\mu \mathrm{L}, 1.05)$. A colorless foam (172 mg, 33\%). $\quad{ }^{1} \mathrm{H}$ NMR (400 MHz, $\left.\mathrm{CDCl}_{3} / \mathrm{TMS}\right) \delta 13.03$ (bs, $1 \mathrm{H}$ ), 8.30 (d, 2H, $J=7.7$ Hz), 7.69-7.67 (m, 3H), 7.63 (d, 2H, $J=6.9 \mathrm{~Hz}), 7.57-7.55$ (m, 3H), 7.48-7.30 (m, 11H), 6.34 (dd, 1H, $J=8.4,5.8 \mathrm{~Hz}$ ), 5.16 (d, 1H, $J=4.0 \mathrm{~Hz}$ ), 5.09 (dd, $1 \mathrm{H}, J=8.8,4.0$ Hz), 4.27 (ddd, 1H, $J=8.8,3.6,3.2 \mathrm{~Hz}$ ), 4.03 (dd, 1H, $J=11.6,3.2 \mathrm{~Hz}$ ), 3.76 (dd, $1 \mathrm{H}, J$ 
= 11.6, 3.6 Hz), 3.05 (dd, $1 \mathrm{H}, J=14.2,5.8 \mathrm{~Hz}), 2.49$ (dd, $1 \mathrm{H}, J=14.2,8.4 \mathrm{~Hz}), 2.07$ (s,

3H), 1.03 (s, 9H). ${ }^{13} \mathrm{C}$ NMR (100 MHz, $\left.\mathrm{CDCl}_{3}\right) \delta 170.1,152.4,135.6,133.1,130.1$, 129.8, 128.7, 128.4, 128.1, 127.7, 125.2, 92.3, 88.6, 86.8, 80.8, 73.1, 62.5, 48.8, 26.8, 20.7, 19.8. FTIR (film) 3200, 3067, 2939, 2858, 1743, 1713, 1593, 1246, $1113 \mathrm{~cm}^{-1}$. HRMS (ESIMS) m/z calcd for $\mathrm{C}_{42} \mathrm{H}_{43} \mathrm{~N}_{3} \mathrm{O}_{7} \mathrm{SiF}(\mathrm{M}+\mathrm{H})^{+}$748.2849, found 748.2846.

\section{Deprotection}

(1'S,3'R,4'R,5'R,7'S)-1-\{4'-Hydroxy-3'-hydroxymethyl-1'-phenyl-2',6'-dioxabicycl o[3.3.0]oct-7'-yl\}-5-fluorouracil (WNA- $\boldsymbol{\beta} F \mathbf{U}, \mathbf{1 7 b})$. A colorless foam (92\%). ${ }^{1} \mathrm{H}$ NMR (400 MHz, CD 3 OD) $\delta 8.12$ (d, 1H, $J=6.6 \mathrm{~Hz}$ ), 7.68 (d, 2H, $J=7.9 \mathrm{~Hz}$ ), 7.35 (dd, 2H, $J=7.9,7.3 \mathrm{~Hz}$ ), 7.26 (t, 1H, $J=7.3 \mathrm{~Hz}), 6.27$ (dd, $1 \mathrm{H}, J=8.6,5.8 \mathrm{~Hz}), 4.85$ (d, 1H, $J=3.6 \mathrm{~Hz}$ ), 4.00 (ddd, 1H, $J=8.7,5.8,2.6 \mathrm{~Hz}), 3.90-3.86$ (m, 2H), 3.67 (dd, 1H, $J=$ 12.2, $5.8 \mathrm{~Hz}$ ), 2.78 (dd, $1 \mathrm{H}, J=13.9,5.8 \mathrm{~Hz}), 2.66$ (dd, $1 \mathrm{H}, J=13.9,8.6 \mathrm{~Hz}) .{ }^{13} \mathrm{C}$ NMR (125 MHz, CD 3 OD) $\delta 159.6,150.7,142.9,141.5,129.4,128.7,127.0,126.6,93.1$, 90.6, 90.2, 84.7, 73.7, 63.5, 49.1. FTIR (KBr) 3389, 3231, 3061, 2930, 2825, 1697, 1666, 1359, 1272, $1059 \mathrm{~cm}^{-1}$. HRMS (ESIMS) m/z calcd for $\mathrm{C}_{17} \mathrm{H}_{17} \mathrm{~N}_{2} \mathrm{O}_{6} \mathrm{FNa}(\mathrm{M}+$ $\mathrm{Na})^{+}$387.0963, found 387.0972. 
(1'S,3'R,4'R,5'R,7’S)- $N^{4}$-Benzoyl-1-\{4'-Hhydroxy-3'-hydroxymethyl-1'-phenyl-2',6

'-dioxabicyclo[3.3.0]oct-7'-yl\}-5-bromocytosine (WNA- $\beta B r C, 18 b)$ A colorless foam (89\%). ${ }^{1} \mathrm{H}$ NMR (400 MHz, $\left.\mathrm{CD}_{3} \mathrm{OD}\right) \delta 8.41$ (bs, 1H), 8.28 (bs, 2H), 7.70 (d, 2H, $J=7.8 \mathrm{~Hz}$ ), 7.58 (bs, 1H), 7.47 (bs, 2H), 7.32 (dd, 2H, $J=7.8,7.3 \mathrm{~Hz}), 7.26$ (t, 1H, $J=$ $7.3 \mathrm{~Hz}$ ), 6.24 (dd, 1H, $J=7.3,6.7 \mathrm{~Hz}$ ), 4.94 (d, 1H, $J=3.2 \mathrm{~Hz}$ ), 4.03 (ddd, 1H, $J=8.6$, 6.0, $3.6 \mathrm{~Hz}), 3.92-3.87$ (m, 2H), 3.69 (dd, 1H, $J=12.2,6.0 \mathrm{~Hz}), 2.92-2.83(\mathrm{~m} 1 \mathrm{H})$, 2.78-2.72 (m, 1H). ${ }^{13} \mathrm{C}$ NMR (100 MHz, $\left.\mathrm{CD}_{3} \mathrm{OD}\right) \delta$ 144.6, 141.4, 134.1, 129.4, 128.7, 127.7, 126.6, 93.3, 92.2, 91.2, 84.5, 73.7, 63.5, 50.1. FTIR (film) 3379, 3065, 2935, 2881, 1771, 1626, 1558, 1450, 1348, $1277 \mathrm{~cm}^{-1}$. HRMS (ESIMS) m/z calcd for $\mathrm{C}_{24} \mathrm{H}_{22} \mathrm{~N}_{3} \mathrm{O}_{6} \mathrm{BrNa}(\mathrm{M}+\mathrm{Na})^{+}$550.0584, 552.0568, found 550.0559, 552.0608.

(1'S,3'R,4'R,5'R,7'S)-N $N^{4}$-Benzoyl-1-\{4'-hydroxy-3'-hydroxymethyl-1'-phenyl-2',6'dioxabicyclo[3.3.0]oct-7'-yl\}-5-fluorocytosine (WNA- $\beta$ FC, 19b). A colorless foam (85\%). $\quad{ }^{1} \mathrm{H}$ NMR (400 MHz, CD $\left.3 \mathrm{OD}\right) \delta 8.43$ (d, 1H, $J=5.2 \mathrm{~Hz}$ ), 8.04 (d, 2H, $J=7.5$ Hz), 7.68 (d, 2H, $J=7.8 \mathrm{~Hz}), 7.62$ (t, 1H, $J=7.9 \mathrm{~Hz}$ ), 7.62 (t, 1H, $J=7.9 \mathrm{~Hz}), 7.50$ (dd, 2H, $J=7.9,7.5 \mathrm{~Hz}$ ), 7.35 (dd, 2H, $J=7.8,7.3 \mathrm{~Hz}$ ), 7.26 (t, 1H, $J=7.3 \mathrm{~Hz}$ ), 6.29 (dd, $1 \mathrm{H}, J=8.2,5.6 \mathrm{~Hz}), 4.93$ (d, 1H, $J=3.8 \mathrm{~Hz}$ ), 4.04 (ddd, 1H, $J=8.6,5.8,2.6 \mathrm{~Hz}$ ), 
3.93-3.88 (m, 2H), 3.69 (dd, 1H, $J=12.2,5.8 \mathrm{~Hz}$ ), 3.00 (dd, 1H, $J=13.9,5.6 \mathrm{~Hz}), 2.67$

(dd, $1 \mathrm{H}, J=13.9,8.2 \mathrm{~Hz}) .{ }^{13} \mathrm{C}$ NMR $\left(100 \mathrm{MHz}, \mathrm{CD}_{3} \mathrm{OD}\right) \delta 141.5,134.3,130.2,129.7$, 129.4, 128.7, 126.6, 93.2, 91.6, 91.2, 84.5, 73.7, 63.4, 49.9. FTIR (film) 3281, 3067, 2882, 2830, 1703, 1670, 1566, 1472, 1339, $1250 \mathrm{~cm}^{-1}$. HRMS (ESIMS) m/z calcd for $\mathrm{C}_{24} \mathrm{H}_{22} \mathrm{~N}_{3} \mathrm{O}_{6} \mathrm{FNa}(\mathrm{M}+\mathrm{Na})^{+}$490.1385, found 490.1344 .

(1'S,3'R,4'R,5'R,7'S)-1-\{4'-Hydroxy-3'-hydroxymethyl-1'-phenyl-2',6'-dioxabicycl o[3.3.0]oct-7'-yl\}-uracil (WNA- $\beta \mathbf{U}, 21 \mathbf{2}) . \quad \mathrm{NaHSO}_{3}(833 \mathrm{mg}, 8.01 \mathrm{mmol})$ and $\mathrm{Na}_{2} \mathrm{SO}_{3}$ (67 mg, $\left.0.534 \mathrm{mmol}\right)$ were added to a solution of $\mathbf{2 0}^{22}(120 \mathrm{mg}, 0.267 \mathrm{mmol})$ in water / dioxane $=1: 1(10 \mathrm{~mL})$ at $80^{\circ} \mathrm{C}$. The mixture was stirred for $24 \mathrm{~h}$ and cooled to room temperature. A solution of $10 \% \mathrm{NaOH}(6.0 \mathrm{~mL})$ was added to the mixture and stirred for 5 min. The mixture was diluted with $\mathrm{MeOH}$ and filtered through a Celite545 pad, and then the filtrate was evaporated. The residue was purified by flash chromatography (silica gel, $\mathrm{CH}_{3} \mathrm{Cl} / \mathrm{MeOH}=19: 1$ ) to give $\mathbf{2 1 b}$ as a colorless foam (88.4 mg, 0.256 mmol, 96\%). $\quad{ }^{1} \mathrm{H}$ NMR (400 MHz, CD $\left.{ }_{3} \mathrm{OD}\right) \delta 7.88$ (d, $\left.1 \mathrm{H}, J=8.1 \mathrm{~Hz}\right), 7.68$ (d, 2H, $J=8.2 \mathrm{~Hz}$ ), 7.35 (dd, 2H, $J=8.2,7.1 \mathrm{~Hz}), 7.26$ (t, 1H, $J=7.1 \mathrm{~Hz}), 6.29$ (dd, 1H, $J=8.2,6.0 \mathrm{~Hz}$ ), 5.75 (d, 1H, $J=8.1 \mathrm{~Hz}$ ), 4.83 (d, 1H, $J=3.8 \mathrm{~Hz}$ ), 4.01 (ddd, 1H, $J=$ 
8.7, 5.8, $2.4 \mathrm{~Hz}$ ), 3.90-3.85 (m, 2H), 3.68 (dd, 1H, $J=12.3,5.8 \mathrm{~Hz}), 2.79$ (dd, 1H, $J=$ 13.9, $6.0 \mathrm{~Hz}$ ), 2.70 (dd, $1 \mathrm{H}, J=13.9,8.2 \mathrm{~Hz}) .{ }^{13} \mathrm{C}$ NMR (125 MHz, CD $\left.{ }_{3} \mathrm{OD}\right) \delta 166.2$, 152.1, 143.2, 141.6, 129.4, 128.7, 126.5, 103.0, 93.2, 90.8, 90.5, 84.6, 73.7, 63.5, 49.0. FTIR (KBr) 3430, 3242, 2926, 1681, 1636, 1558, 1463, 1278, $1070 \mathrm{~cm}^{-1}$. HRMS (ESIMS) $\mathrm{m} / \mathrm{z}$ calcd for $\mathrm{C}_{17} \mathrm{H}_{18} \mathrm{~N}_{2} \mathrm{O}_{6} \mathrm{Na}(\mathrm{M}+\mathrm{Na})^{+}$369.1057, found 369.1009.

Synthesis of the $\beta$-cyanoethylphosphoramidite precursors

(1'S,3'R,4'R,5'R,7'S)-1-\{4'-(2-Cyanoethyl- $N$, $N$-diisopropylphosphoramidityloxy)-3

'-dimethoxytrithyloxymethyl-1'-phenyl-2',6'-dioxabicyclo[3.3.0]oct-7'-yl\}-5-fluoro

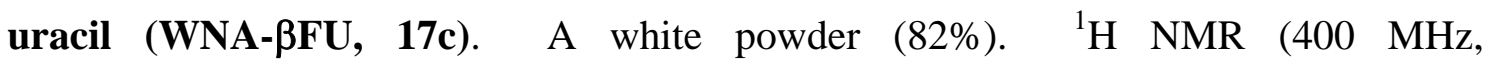
$\left.\mathrm{CDCl}_{3} / \mathrm{TMS}\right) \delta 7.74(\mathrm{~d}, 0.4 \mathrm{H}, J=6.0 \mathrm{~Hz}), 7.61(\mathrm{~d}, 1.2 \mathrm{H}, J=8.2 \mathrm{~Hz}), 7.56(\mathrm{~d}, 0.8 \mathrm{H}, J=$ $8.2 \mathrm{~Hz}), 7.53(\mathrm{~d}, 0.6 \mathrm{H}, J=6.0 \mathrm{~Hz}), 7.45(\mathrm{~d}, 0.8 \mathrm{H}, J=7.3 \mathrm{~Hz}), 7.43$ (d, $1.2 \mathrm{H}, J=7.3$ Hz), 7.35-7.20 (m, 10H), 6.81 (d, 4H, $J=8.7 \mathrm{~Hz}), 6.37$ (dd, 0.4H, $J=7.8,6.0 \mathrm{~Hz}), 6.24$ (dd, 0.6H, $J=7.3,6.4 \mathrm{~Hz}$ ), 5.05 (d, 0.4H, $J=3.7 \mathrm{~Hz}$ ), 4.91 (d, 0.6H, $J=3.7 \mathrm{~Hz}$ ), 4.39-4.12 (m, 2H), 3.80 (s, 3.6H), 3.79 (s, 2.4H), 3.85-3.68 (m, 1H), 3.57-3.37 (m, 4H), 3.24-3.19 (m, 1H), 3.09-3.02 (m, 1H), 2.64-2.30 (m, 3H), 1.07 (d, 2H, $J=6.4 \mathrm{~Hz}$ ), 0.97 (d, 4H, $J=6.9 \mathrm{~Hz}), 0.91(\mathrm{~d}, 2 \mathrm{H}, J=6.4 \mathrm{~Hz}), 0.88(\mathrm{~d}, 4 \mathrm{H}, J=6.9 \mathrm{~Hz}) . \quad{ }^{31} \mathrm{P}$ NMR 
(161 MHz, $\left.\mathrm{CDCl}_{3}\right) \delta 150.1,149.5 . \quad$ FTIR (film) 3192, 3070, 2966, 2837, 1699, 1607, 1510, $1464 \mathrm{~cm}^{-1}$. $\quad \operatorname{ESIMS~(m/z)~} 867[\mathrm{M}+\mathrm{H}]^{+}, 889[\mathrm{M}+\mathrm{Na}]^{+}$.

(1'S,3'R,4'R,5'R,7'S)- $N^{4}$-Benzoyl-1-\{4'-(2-cyanoethyl- $N$, $N$-diisopropylphosphorami dityloxy)-3'-dimethoxytrithyloxymethyl-1'-phenyl-2',6'-dioxabicyclo[3.3.0]oct-7'-y 1)-5-bromocytosine (WNA- $\beta$ BrC, 18c). A white powder (84\%). ${ }^{1} \mathrm{H}$ NMR (400 MHz, $\left.\mathrm{CDCl}_{3} / \mathrm{TMS}\right) \delta 13.26$ (bs, 1H), 8.36 (d, 2H, $J=6.1 \mathrm{~Hz}$ ), 8.10 (s, 0.5H), 7.92 (s, $0.5 \mathrm{H}), 7.64-7.54$ (m, 3H), 7.48-7.43 (m, 4H), 7.36-7.25 (m, 9H), 7.23-7.19 (m, 1H), 6.83-6.80 (m, 4H), 6.37 (dd, 0.5H, $J=7.9,6.1 \mathrm{~Hz}$ ), 6.22 (dd, 0.5H, $J=7.9,6.1 \mathrm{~Hz}$ ), $5.11(\mathrm{~d}, 0.5 \mathrm{H}, J=3.7 \mathrm{~Hz}), 4.96(\mathrm{~d}, 0.5 \mathrm{H}, J=3.1 \mathrm{~Hz}), 4.42-4.13(\mathrm{~m}, 2 \mathrm{H}), 3.80(\mathrm{~s}, 3 \mathrm{H})$, 3.79 (s, 3H), 3.76-3.40 (m, 5H), 3.25-3.10 (m, 2H), 2.63-2.30 (m, 3H), 1.15-1.08 (m, 6H), 0.93-0.87 (m, 6H). ${ }^{31} \mathrm{P}$ NMR (161 MHz, $\left.\mathrm{CDCl}_{3}\right) \delta 150.3$, 149.4. $\quad$ FTIR (film) 3163, 3067, 2970, 2836, 1715, 1574, 1506, 1448, 1340, 1250, 1175, $1034 \mathrm{~cm}^{-1}$. ESIMS (m/z) 1030.32, $1032.25[\mathrm{M}+\mathrm{H}]^{+}$.

(1'S,3'R,4'R,5'R,7'S)-N $N^{4}$-Benzoyl-1-\{4'-(2-cyanoethyl- $N$, $N$-diisopropylphosphorami dityloxy)-3'-dimethoxytrithyloxymethyl-1'-phenyl-2',6'-dioxabicyclo[3.3.0]oct-7'-y l\}-5-fluorocytosine (WNA- $\beta$ FC, 19c). A white powder (87\%). $\quad{ }^{1} \mathrm{H}$ NMR (400 MHz, 
$\left.\mathrm{CDCl}_{3} / \mathrm{TMS}\right) \delta 8.30(\mathrm{~d}, 2 \mathrm{H}, J=7.5 \mathrm{~Hz}), 7.89(\mathrm{~d}, 0.5 \mathrm{H}, J=5.6 \mathrm{~Hz}), 7.68(\mathrm{~d}, 0.5 \mathrm{H}, J=$ 5.6 Hz), 7.63-7.15 (m, 17H), 6.83-6.80 (m, 4H), 6.40 (dd, 0.5H, $J=7.1,6.7 \mathrm{~Hz}), 6.27$ (dd, 0.5H, $J=7.3,6.6 \mathrm{~Hz}$ ), 5.07 (d, 0.5H, $J=3.8 \mathrm{~Hz}$ ), 4.93 (d, 0.5H, $J=3.6 \mathrm{~Hz}$ ), 4.41-4.11 (m, 2H), 3.80 (s, 3H), 3.79 (s, 3H), 3.75-3.40 (m, 5H), 3.25-3.20 (m, 1H), 3.15-3.09 (m, 1H), 2.66-2.28 (m, 3H), $1.14(\mathrm{~d}, 2 \mathrm{H}, J=6.7 \mathrm{~Hz}), 1.08(\mathrm{~d}, 4 \mathrm{H}, J=6.7$ $\mathrm{Hz}), 0.91(\mathrm{~d}, 2 \mathrm{H}, J=6.7 \mathrm{~Hz}), 0.88(\mathrm{~d}, 4 \mathrm{H}, J=6.7 \mathrm{~Hz}) .{ }^{31} \mathrm{P} \mathrm{NMR}\left(161 \mathrm{MHz}, \mathrm{CDCl}_{3}\right) \delta$ 150.1, 149.5. FTIR (film) 3192, 3070, 2962, 2836, 1717, 1593, 1506, 1448, 1339, 1250, 1177, $1034 \mathrm{~cm}^{-1} . \quad \operatorname{ESIMS}(\mathrm{m} / \mathrm{z}) 970.37[\mathrm{M}+\mathrm{H}]^{+}$.

(1'S,3'R,4'R,5'R,7'S)-1-\{4'-(2-cyanoethyl- $N, N$-diisopropylphosphoramidityloxy)-3' -dimethoxytrithyloxymethyl-1'-phenyl-2',6'-dioxabicyclo[3.3.0]oct-7'-yl\}-uracil

(WNA- $\beta \mathbf{U}, 21 \mathrm{2}) . \quad$ A white powder (53\%). ${ }^{1} \mathrm{H}$ NMR (400 $\left.\mathrm{MHz}, \mathrm{CDCl}_{3} / \mathrm{TMS}\right) \delta$ 7.65-7.59 (m, 2H), 7.52-7.41 (m, 3H), 7.35-7.28 (m, 9H), 7.25-7.20 (m, 1H), 6.83-6.80 (m, 4H), 6.37 (dd, 0.4H, $J=7.9,6.1 \mathrm{~Hz}$ ), 6.22 (dd, 0.6H, $J=7.9,6.1 \mathrm{~Hz}$ ), 5.83 (d, 0.4H, $J=7.9 \mathrm{~Hz}), 5.78$ (d, 0.6H, $J=8.5 \mathrm{~Hz}), 5.01$ (d, 0.4H, $J=3.7 \mathrm{~Hz}), 4.91$ (d, 0.6H, $J=3.7 \mathrm{~Hz}), 4.37-4.09$ (m, 2H), 3.80 (s, 3.6H), 3.79 (s, 2.4H), 3.77-3.36 (m, 5H), 3.24-3.19 (m, 1H), 3.08-2.92 (m, 1H), 2.69-2.28 (m, 3H), 1.13 (d, 4H, $J=6.7 \mathrm{~Hz}$ ), 
1.07 (d, 2H, $J=6.7 \mathrm{~Hz}), 1.05$ (d, 4H, $J=6.7 \mathrm{~Hz}), 0.89$ (d, 2H, $J=6.7 \mathrm{~Hz}) . \quad{ }^{31} \mathrm{P}$ NMR (161 MHz, $\left.\mathrm{CDCl}_{3}\right) \delta 150.1,149.3 . \quad$ FTIR (film) 3184, 3059, 2966, 2872, 1684, 1609, 1508, $1448 \mathrm{~cm}^{-1}$. $\quad \operatorname{ESIMS~(m/z)~} 849[\mathrm{M}+\mathrm{H}]^{+}, 871[\mathrm{M}+\mathrm{Na}]^{+}$.

Synthesis of $p \mathrm{Br}-\mathrm{WNA}-\beta \mathrm{C}, \mathrm{mBr}-, \mathrm{oBr}-\mathrm{WNA}-\beta \mathrm{T}, \boldsymbol{\beta C}$ (Scheme 2 )

(1RS,2R,3R,4R)-1-m-Bromophenyl-5-O-(tert-butyldiphenylsilyl)-2,3-O-isopropylide

neribose $(\mathbf{2 4}(\boldsymbol{m}))$. By a similar procedure as described for the synthesis of $\mathbf{2 5 ( p )}$ with the use of ribonolactone $\mathbf{2 4}(8.0 \mathrm{~g}, 18.8 \mathrm{mmol}), \mathbf{2 4}(\mathbf{m})$ was obtained as a colorless oil (8.73 g, 15.0 mmol, 80\%). ${ }^{1} \mathrm{H}$ NMR (400 MHz, CDCl $/$ TMS) $\delta 7.77$ (s, 1H), 7.70 (dd, 2H, $J=8.0,1.4 \mathrm{~Hz}$ ), 7.66 (dd, 2H, $J=8.0,1.4 \mathrm{~Hz}), 7.55-7.52(\mathrm{~m}, 1 \mathrm{H}), 7.48-7.36$ (m, 7H), 7.23 (t, $0.8 \mathrm{H}, J=7.8 \mathrm{~Hz}$ ), 7.17 (t, 0.2H, $J=7.8 \mathrm{~Hz}$ ), 4.93 (dd, 0.2H, $J=7.3,4.4$ Hz), 4.90 (d, 0.8H, $J=5.7,1.4 \mathrm{~Hz}), 4.70$ (s, 0.8H), 4.64 (d, 0.8H, $J=5.7 \mathrm{~Hz}), 4.59$ (d, 0.2H, $J=7.1 \mathrm{~Hz}$ ), 4.49 (s, 0.2H), 4.44-4.33 (m, 0.8H), 4.32-4.31 (m, 0.2H), 4.00 (dd, 0.2H, $J=14.4,3.2 \mathrm{~Hz}$ ), $3.94(\mathrm{dd}, 0.8 \mathrm{H}, J=14.2,3.2 \mathrm{~Hz}$ ), 3.90 (dd, $0.2 \mathrm{H}, J=14.4,3.2$ Hz), 3.76 (dd, 0.8H, $J=14.2,3.2 \mathrm{~Hz}$ ), 1.38 (s, 3H), 1.25 (s, 3H), 1.12 (s, 9H). FTIR (film) $3350 \mathrm{~cm}^{-1}$. HRMS (ESIMS) m/z calcd for $\mathrm{C}_{30} \mathrm{H}_{35} \mathrm{O}_{5} \mathrm{BrSiNa}(\mathrm{M}+\mathrm{Na})^{+}$605.1329, 607.1314, found 605.1378, 607.1274. 
(1RS,2R,3R,4R)-1-o-Bromophenyl-5-O-(tert-butyldiphenylsilyl)-2,3-O-isopropylide

neribose (25(o)). A solution of $n$-BuLi (1.6 Min hexane, $29.0 \mathrm{~mL}, 45.0 \mathrm{mmol}$ ) was added slowly to a solution of 1,2-dibromobenzene $(5.8 \mathrm{~mL}, 15.0 \mathrm{mmol})$ in ether/THF = 1:1 (90 mL) at $-110^{\circ} \mathrm{C}$ in portions. After stirring for $1 \mathrm{~h}$ at $-110^{\circ} \mathrm{C}$, a solution of 22 (3.2 g, $7.5 \mathrm{mmol}$ ) in THF (24 mL) was added to the mixture. The reaction mixture was stirred for $3.5 \mathrm{~h}$, and quenched with sat. $\mathrm{NH}_{4} \mathrm{Cl}$ solution, allowed to warm to $0^{\circ} \mathrm{C}$, and extracted with AcOEt. The organic layer was successively washed with water and brine, dried over $\mathrm{Na}_{2} \mathrm{SO}_{4}$, and evaporated. The residue was purified by flash chromatography (silica gel, hexane/AcOEt $=10: 1)$ to give $\mathbf{2 5}(\mathbf{o})$ as a colorless oil (4.4 g, 7.5 mmol, quantitative). $\quad{ }^{1} \mathrm{H}$ NMR (400 MHz, $\left.\mathrm{CDCl}_{3} / \mathrm{TMS}\right) \delta 7.96$ (dd, 0.2H, $J=8.0$, $1.6 \mathrm{~Hz}$ ), 7.80 (dd, 0.8H, $J=7.9,1.6 \mathrm{~Hz}), 7.70-7.57$ (m, 5H), 7.44-7.36 (m, 6H), 7.27-7.23 (m, 1H), 7.15-7.13 (m, 1H), $5.26(\mathrm{~d}, 0.8 \mathrm{H}, J=5.8 \mathrm{~Hz}), 4.98$ (d, 0.2H, $J=7.1$ Hz), 4.96 (s, 0.2H), 4.84 (d, 0.8H, $J=5.8 \mathrm{~Hz}$ ), 4.77 (dd, 0.2H, $J=7.1,4.9 \mathrm{~Hz}$ ), 4.41-4.39 (m, 0.8H), 4.34-4.31 (m, 0.2H), 4.26 (s, 0.8H), 3.94 (dd, 0.8H, $J=11.0,4.0$ Hz), 3.92 (dd, 0.2H, $J=10.8,4.3 \mathrm{~Hz}$ ), 3.88 (dd, 0.2H, $J=10.8,4.3 \mathrm{~Hz}$ ), 3.76 (dd, 0.8H, $J=11.0,4.0 \mathrm{~Hz}$ ), 1.53 (s, 3H), 1.24 (s, 3H), 1.09 (s, 9H). FTIR (film) 3390, 1589 
$\mathrm{cm}^{-1}$. ESIMS (m/z) 565, $567 \quad[\mathrm{M}-\mathrm{OH}]^{+}$. HRMS (ESIMS) m/z calcd for $\mathrm{C}_{30} \mathrm{H}_{35} \mathrm{O}_{5} \mathrm{BrSiNa}(\mathrm{M}+\mathrm{Na})^{+}$605.1329, 607.1314, found 605.1379, 607.1320.

$(1 R, 3 R, 4 R, 5 R, 7 R S)-1-m$-Bromophenyl-3-(tert-butyldiphenylsilyloxymethyl)-4,7-dia cetoxy-2,6-dioxabicyclo[3.3.0]octane $(27(m))$. By a similar procedure as described for the synthesis of $\mathbf{2 6}(\boldsymbol{p})$ with the use of $\mathbf{2 6 ( \boldsymbol { m } )}(7.68 \mathrm{~g}, 13.2 \mathrm{mmol}), \mathbf{2 7}(\mathbf{m})$ was prepared as a colorless foam (1.08 g, $1.65 \mathrm{mmol}) . \quad{ }^{1} \mathrm{H}$ NMR (400 MHz, $\left.\mathrm{CDCl}_{3} / \mathrm{TMS}\right) \delta$ 7.79-7.60 (m, 6H), 7.46-7.34 (m, 7H), 7.18 (t, 1H, $J=7.9 \mathrm{~Hz}), 6.62$ (d, 0.9H, $J=5.3$ Hz), $6.48(\mathrm{~d}, 0.1 \mathrm{H}, J=5.7 \mathrm{~Hz}), 5.01-4.97(\mathrm{~m}, 1 \mathrm{H}, J=9.6,4.5 \mathrm{~Hz}), 4.93$ (d, 0.1H, $J=$ $5.2 \mathrm{~Hz}), 4.82(\mathrm{~d}, 0.9 \mathrm{H}, J=4.5 \mathrm{~Hz}), 4.50(\mathrm{dt}, 0.1 \mathrm{H}, J=8.8,3.2 \mathrm{~Hz}), 4.20$ (dt, 0.9H, $J=$ 9.2, $2.6 \mathrm{~Hz}$ ), 4.06 (dd, 0.9H, $J=11.8,2.3 \mathrm{~Hz}$ ), 4.03 (dd, 0.1H, $J=11.6,3.2 \mathrm{~Hz}$ ), 3.76 (dd, 0.9H, $J=11.8,3.4 \mathrm{~Hz}$ ), 3.74 (dd, 0.1H, $J=11.6,3.2 \mathrm{~Hz}$ ), 2.82 (dd, 0.9H, $J=15.2$, $5.3 \mathrm{~Hz}$ ), 2.67 (dd, 0.1H, $J=15.0,5.7 \mathrm{~Hz}$ ), 2.58 (d, 1H, $J=15.2 \mathrm{~Hz}$ ), 2.16 (s, 3H), 2.04 (s, 3H), 1.05 (s, 5H), 0.98 (s, 4H). FTIR (film) 2927, 2856, 1825, 1740, 1690, 1632, 1451, $1426 \mathrm{~cm}^{-1}$. HRMS (ESIMS) $\mathrm{m} / \mathrm{z}$ calcd for $\mathrm{C}_{33} \mathrm{H}_{37} \mathrm{O}_{7} \mathrm{SiBrNa}(\mathrm{M}+\mathrm{Na})^{+}$ 675.1384, 677.1370, found 675.1433, 677.1373. 
(1R,3R,4R,5R,7RS)-1-o-Bromophenyl-3-(tert-butyldiphenylsilyloxymethyl)-4,7-diac

etoxy-2,6-dioxabicyclo[3.3.0]octane (28(o)). By a similar procedure as described for the synthesis of $\mathbf{2 6 ( p )}$ with use of $\mathbf{2 5 ( o )}(6.1 \mathrm{~g}, 10.5 \mathrm{mmol}), \mathbf{2 8 ( o )}$ was prepared as a colorless foam (663 mg, $1.02 \mathrm{mmol}) . \quad{ }^{1} \mathrm{H}$ NMR (400 MHz, CDCl $/$ TMS) $\delta 8.00$ (d, 0.5H, $J=1.9 \mathrm{~Hz}), 7.87(\mathrm{~d}, 0.5 \mathrm{H}, J=1.9 \mathrm{~Hz}), 7.69-7.31(\mathrm{~m}, 11 \mathrm{H}), 7.22-7.09(\mathrm{~m}, 2 \mathrm{H})$, $6.58(\mathrm{~d}, 0.5 \mathrm{H}, J=6.4 \mathrm{~Hz}), 6.54(\mathrm{dd}, 0.5 \mathrm{H}, J=6.0,4.1 \mathrm{~Hz}), 5.59(\mathrm{~d}, 0.5 \mathrm{H}, J=3.7 \mathrm{~Hz})$, 5.38 (d, 0.5H, $J=5.1 \mathrm{~Hz}$ ), 5.02 (dd, 0.5H, $J=9.2,3.7 \mathrm{~Hz}$ ), 4.92 (dd, $0.5 \mathrm{H}, J=9.2,5.3$ Hz), 4.53-4.51 (m, 0.5H), 4.24-4.22 (m, 0.5H), 4.06 (dd, 0.5H, $J=11.8,3.2 \mathrm{~Hz}), 3.96$ (dd, 0.5H, $J=11.6,3.0 \mathrm{~Hz}$ ), 3.76 (dd, 0.5H, $J=11.8,3.2 \mathrm{~Hz}$ ), 3.68 (dd, 0.5H, $J=11.6$, $3.4 \mathrm{~Hz}$ ), 3.38 (dd, 0.5H, $J=15.0,6.4 \mathrm{~Hz}$ ), 3.00 (dd, 0.5H, $J=15.0,4.1 \mathrm{~Hz}$ ), 2.88 (dd, 0.5H, $J=15.0,6.0 \mathrm{~Hz}$ ), 2.36 (d, 0.5H, $J=15.0 \mathrm{~Hz}$ ), 2.07 (s, 3H), 2.03 (s, 3H), 0.99 (s, 4H), 0.94 (s, 5H). FTIR (film) 1749, $1463 \mathrm{~cm}^{-1}$. HRMS (ESIMS) m/z calcd for $\mathrm{C}_{33} \mathrm{H}_{37} \mathrm{O}_{7} \mathrm{SiBrNa}(\mathrm{M}+\mathrm{Na})^{+}$675.1384, 677.1370, found 675.1397, 677.1394.

(1'S,3'R,4'R,5'R,7'S)-\{4'-Acetoxy-1'-m-Bromophenyl-3'-(tert-butyldiphenylsilylox ymethyl)-2',6'-dioxabicyclo[3.3.0]oct-7'-yl\}-thymine $\quad(m B r-W N A-\beta T, \quad 30 a(m))$ : Reagents: $\quad$ 27(m) (310 mg, $0.47 \mathrm{mmol})$, thymine (89.5 mg, $0.71 \mathrm{mmol})$, BSA (0.35 mL, 
$1.42 \mathrm{mmol})$, TMSOTf $(0.13 \mathrm{~mL}, 0.71 \mathrm{mmol}), \mathrm{CH}_{3} \mathrm{CN}(10 \mathrm{~mL})$. The isomers were separated by flash chromatography (silica gel, $\mathrm{CHCl}_{3} /$ hexane/acetone $=2: 4: 1$ ) to give the each isomer in total 99\% yield. $\quad m B r-W N A-\beta T(30 a(m))$ as a colorless foam (186 mg, 0.26 mmol, 55\%). $\quad{ }^{1} \mathrm{H}$ NMR (400 MHz, $\left.\mathrm{CDCl}_{3} / \mathrm{TMS}\right) \delta 8.65$ (bs, 1H), 7.66-7.53 (m, 6H), 7.45-7.20 (m, 9H), 6.27 (dd, 1H, $J=8.2,5.8 \mathrm{~Hz}$ ), 5.13 (d, 1H, $J=4.3 \mathrm{~Hz}$ ), 5.04 (dd, 1H, $J=8.6,4.3 \mathrm{~Hz}), 4.27-4.24$ (m, 1H), 3.99 (dd, 1H, $J=11.6,2.8 \mathrm{~Hz}$ ), 3.73 (dd, 1H, $J=11.6,3.6 \mathrm{~Hz}$ ), 2.88 (dd, 1H, $J=14.0,5.8 \mathrm{~Hz}$ ), 2.49 (dd, $1 \mathrm{H}, J=14.0,8.2$ Hz), 2.05 (s, 3H), 1.99 (s, 3H), 1.02 (s, 9H). ${ }^{13} \mathrm{C}$ NMR (100 MHz, $\left.\mathrm{CDCl}_{3}\right) \delta$ 170.1, 163.4, 148.3, 142.2, 135.6, 135.6, 132.9, 131.1, 130.3, 129.8, 128.3, 127.8, 127.7, 124.0, 122.8, 111.6, 91.8, 88.3, 86.2, 80.9, 72.9, 62.4, 48.4, 26.8, 20.7, 19.2, 12.6. FTIR (film) 2940, 1690, 1674, $1550 \mathrm{~cm}^{-1}$. ESIMS (m/z) 719, $721(\mathrm{M}+\mathrm{H})^{+}$, HRMS (ESIMS) $\mathrm{m} / \mathrm{z}$ calcd for $\mathrm{C}_{36} \mathrm{H}_{39} \mathrm{~N}_{2} \mathrm{O}_{7} \mathrm{SiBrNa}(\mathrm{M}+\mathrm{Na})^{+} 741.1602$, 743.1589, found 741.1625, 743.1600.

(1'S,3'R,4'R,5'R,7’S)-\{4'-Acetoxy-1'-o-Bromophenyl-3'-(tert-butyldiphenylsilyloxy methyl)-2',6'-dioxabicyclo[3.3.0]oct-7'-yl\}-thymine $\quad($ oBr-WNA- $\beta$ T, $\quad 31 \mathrm{a}(o))$. Reagents: 28(o) (350 mg, $0.54 \mathrm{mmol})$, thymine (102 mg, $0.80 \mathrm{mmol})$, BSA (0.40 mL, 
$1.61 \mathrm{mmol})$, TMSOTf $(0.19 \mathrm{~mL}, 0.80 \mathrm{mmol}), \mathrm{CH}_{3} \mathrm{CN}(6 \mathrm{~mL})$. The isomers were separated by flash chromatography (silica gel, $\mathrm{CHCl}_{3}$ /hexane/acetone $=2: 4: 1$ ) to give each isomer in total 89\% yield. oBr-WNA- $\beta$ T (31a(o)) as a colorless foam (238 mg, $0.33 \mathrm{mmol}, 62 \%) . \quad{ }^{1} \mathrm{H}$ NMR (500 MHz, $\left.\mathrm{CDCl}_{3} / \mathrm{TMS}\right) \delta 8.06-8.03$ (m, 1H), 7.66-7.58 (m, 7H), 7.45-7.32 (m, 6H), 7.24-7.21 (m, 1H), 7.16-7.13 (m, 1H), 6.46 (dd, 1H, $J=$ 9.1, $5.5 \mathrm{~Hz}$ ), 5.65 (d, 1H, $J=4.1 \mathrm{~Hz}$ ), 4.95 (dd, 1H, $J=9.1,4.1 \mathrm{~Hz}), 4.25-4.22(\mathrm{~m}, 1 \mathrm{H})$, 4.02 (dd, $1 \mathrm{H}, J=11.5,3.0 \mathrm{~Hz}$ ), 3.71 (dd, $1 \mathrm{H}, J=11.5,3.4 \mathrm{~Hz}$ ), 3.00 (dd, $1 \mathrm{H}, J=13.7$, $9.1 \mathrm{~Hz}$ ), 2.64 (dd, 1H, $J=13.7,5.5 \mathrm{~Hz}$ ), 2.03 (s, 3H), 1.98 (s, 3H), 0.98 (s, 9H). ${ }^{13} \mathrm{C}$ NMR $\left(100 \mathrm{MHz}, \mathrm{CDCl}_{3}\right) \delta 170.09,163.34,150.18,137.20,135.62,135.56,135.29$, 134.72, 133.00, 132.79, 129.83, 129.66, 128.06, 127.80, 127.74, 119.53, 111.43, 92.03, 86.43, 82.76, 80.48, 73.16, 62.18, 45.67, 26.79, 20.68, 19.18, 12.73. FTIR (film) 2940, 1744, 1693, $1465 \mathrm{~cm}^{-1}$. HRMS (ESIMS) m/z calcd for $\mathrm{C}_{36} \mathrm{H}_{40} \mathrm{~N}_{2} \mathrm{O}_{7} \mathrm{SiBr}(\mathrm{M}+\mathrm{H})^{+}$ 719.1783, 721.1770, found 719.1614, 721.1768.

(1'S,3'R,4'R,5'R,7'S)-N"-Benzoyl-1-\{4'-Acetoxy-1'-p-Bromophenyl-3'-(tert-butyldi phenylsilyloxymethyl)-2',6'-dioxabicyclo[3.3.0]oct-7'-yl\}-cytosine $\quad(p B r-W N A-\beta C$, 32a(p)). Reagents: 26(p) (239 mg, $0.37 \mathrm{mmol}$ ), $N$-benzoylcytosine (98.4 mg, 0.46 
mmol), BSA (0.22 mL, $0.91 \mathrm{mmol}), \mathrm{SnCl}_{4}(0.09 \mathrm{~mL}, 1.29 \mathrm{mmol}), \mathrm{CH}_{3} \mathrm{CN}(12 \mathrm{~mL})$.

The isomers were separated by flash chromatography (silica gel, $\mathrm{CHCl}_{3} /$ hexane/acetone $=2: 4: 1)$ to give each isomer in total $90 \%$ yield. $p$ Br-WNA- $\beta \mathrm{C}(32 \mathrm{a}(\boldsymbol{p}))$ as a colorless foam (146 mg, $0.18 \mathrm{mmol}, 49 \%) . \quad{ }^{1} \mathrm{H}$ NMR (500 MHz, CDCl 3 /TMS) $\delta 8.67$ (bs, 1H), 7.93-7.88 (m, 3H), 7.64-7.59 (m, 6H), 7.52-7.49 (m, 2H), 7.45-7.33 (m, 10H), 6.30-6.27 (m, 1H), $5.14(\mathrm{~d}, 1 \mathrm{H}, J=4.1 \mathrm{~Hz}), 5.04(\mathrm{dd}, 1 \mathrm{H}, J=8.9,4.1 \mathrm{~Hz}), 4.27-4.24$ (m, 1H), 4.01 (dd, 1H, $J=11.7,2.9 \mathrm{~Hz}), 3.73(\mathrm{dd}, 1 \mathrm{H}, J=11.7,3.4 \mathrm{~Hz}), 3.17$ (dd, $1 \mathrm{H}, J$ = 14.4, $5.6 \mathrm{~Hz}$ ), 2.51 (dd, $1 \mathrm{H}, J=14.4,7.6 \mathrm{~Hz}), 2.03(\mathrm{~s}, 3 \mathrm{H}), 1.01(\mathrm{~s}, 9 \mathrm{H}) .{ }^{13} \mathrm{C} \mathrm{NMR}$ (125 MHz, $\left.\mathrm{CDCl}_{3}\right) \delta 170.0,160.9,138.7,145.0,138.8,135.6,135.6,133.4,133.0$, 132.9, 131.7, 129.9, 129.8, 129.1, 127.8, 127.8, 127.2, 122.0, 92.3, 90.7, 87.2, 80.5, 72.8, 62.4, 49.9, 26.8, 20.7, 19.2. FTIR (film) 3072, 1746, 1664, 1626, $1556 \mathrm{~cm}^{-1}$. HRMS (ESIMS) m/z calcd for $\mathrm{C}_{42} \mathrm{H}_{43} \mathrm{~N}_{3} \mathrm{O}_{7} \mathrm{SiBr}(\mathrm{M}+\mathrm{H})^{+}$808.2048, 810.2038, found 808.2014, 810.2010.

(1'S,3'R,4'R,5'R,7’S)-N"'-Benzoyl-1-\{4'-Acetoxy-1'-m-Bromophenyl-3'-(tert-butyldi phenylsilyloxymethyl)-2',6'-dioxabicyclo[3.3.0]oct-7'-yl\}-cytosine (mBr-WNA- $\beta$ C, 33a(m)). Reagents: $\quad$ 27(m) (390 mg, 0.60 mmol), $N$-benzoylcytosine (152 mg, 0.71 
mmol), BSA (0.34 mL, $1.41 \mathrm{mmol})$, TMSOTf (0.19 mL, $0.83 \mathrm{mmol}), \mathrm{CH}_{3} \mathrm{CN}$ (8 mL).

The isomers were separated by flash chromatography (silica gel, $\mathrm{CHCl}_{3} /$ hexane/acetone $=2: 4: 1)$ to give the each isomer in total $96 \%$ yield. $m \operatorname{Br}-W N A-\beta C(33 a(m))$ as a colorless foam (223 mg, $0.28 \mathrm{mmol}, 46 \%) . \quad{ }^{1} \mathrm{H}$ NMR (500 MHz, $\mathrm{CDCl}_{3} / \mathrm{TMS}$ ) $\delta 8.71$ (bs, 1H), 8.00-7.88 (m, 3H), 7.69-7.59 (m, 7H), 7.52-7.33 (m, 10H), 7.16-7.13 (m, 1H), 6.31 (dd, $1 \mathrm{H}, J=7.6,6.0 \mathrm{~Hz}$ ), 5.18 (d, 1H, $J=4.1 \mathrm{~Hz}$ ), 5.03 (dd, $1 \mathrm{H}, J=8.9,4.1$ Hz), 4.28-4.25 (m, 1H), 4.00 (dd, 1H, $J=11.7,2.9 \mathrm{~Hz}$ ), 3.74 (dd, 1H, $J=11.7,3.6 \mathrm{~Hz}$ ), 3.18 (dd, 1H, $J=14.4,6.0 \mathrm{~Hz}$ ), 2.45 (dd, 1H, $J=14.4,7.6 \mathrm{~Hz}$ ), 2.04 (s, 3H), 1.00 (s, 9H). $\left.{ }^{13} \mathrm{C} \mathrm{NMR} \mathrm{(100} \mathrm{MHz,} \mathrm{CDCl}_{3}\right) \delta 170.0,163.3,144.1,142.2,135.6,135.6,133.3$, 133.0, 132.9, 131.1, 129.8, 129.8, 129.1, 128.3, 127.8, 127.7, 127.6, 124.0, 122.8, 92.0, 90.2, 87.0, 80.6, 72.8, 62.3, 47.9, 26.8, 20.7, 19.1. FTIR (film) 3072, 1745, 1693, 1623, 1553, $1483 \mathrm{~cm}^{-1}$. HRMS (ESIMS) m/z calcd for $\mathrm{C}_{42} \mathrm{H}_{43} \mathrm{~N}_{3} \mathrm{O}_{7} \mathrm{SiBr}(\mathrm{M}+\mathrm{H})^{+}$ 808.2048, 810.2038, found 808.2083, 810.1992.

(1'S,3'R,4'R,5'R,7'S)-N $N^{4}$-Benzoyl-1-\{4'-Acetoxy-1'-o-Bromophenyl-3'-(tert-butyldi phenylsilyloxymethyl)-2',6'-dioxabicyclo[3.3.0]oct-7'-yl\}-cytosine (oBr-WNA- $\beta$ C, 34a(o)). Reagents: 28(o) (281 mg, $0.39 \mathrm{mmol}$ ), $N$-benzoylcytosine (105 mg, 0.49 
mmol), BSA (0.24 mL, $0.98 \mathrm{mmol})$, TMSOTf (0.14 mL, $0.59 \mathrm{mmol}), \mathrm{CH}_{3} \mathrm{CN}$ (10 mL).

The isomers were separated by flash chromatography (silica gel, $\mathrm{CHCl}_{3} /$ hexane/acetone $=2: 4: 1)$ to give each isomer in total $83 \%$ yield. oBr-WNA- $\beta \mathrm{C}(\mathbf{3 4 a} \mathbf{a}(\mathbf{o}))$ as a colorless foam (168 mg, $0.21 \mathrm{mmol}, 53 \%) . \quad{ }^{1} \mathrm{H}$ NMR (500 MHz, $\left.\mathrm{CDCl}_{3} / \mathrm{TMS}\right) \delta 8.62$ (bs, $\left.1 \mathrm{H}\right)$, $8.29(\mathrm{~d}, 1 \mathrm{H}, J=6.6 \mathrm{~Hz}), 8.05(\mathrm{dd}, 1 \mathrm{H}, J=8.0,1.7 \mathrm{~Hz}), 7.88-7.78(\mathrm{~m}, 1 \mathrm{H}), 7.66(\mathrm{dd}, 2 \mathrm{H}$, $J=8.0,1.4 \mathrm{~Hz}$ ), 7.61-7.33 (m, 14H), 7.23-7.20 (m, 1H), 7.15-7.12 (m, 1H), 6.50-6.47 (m, 1H), 5.71 (d, 1H, $J=4.1 \mathrm{~Hz}$ ), 4.95 (dd, 1H, $J=9.3,4.1 \mathrm{~Hz}$ ), 4.27-4.24 (m, 1H), 4.06 (dd, 1H, $J=11.5,3.0 \mathrm{~Hz}$ ), 3.73 (dd, 1H, $J=11.5,3.4 \mathrm{~Hz}$ ), 3.00-2.96 (m, 1H), 2.98 (dd, $1 \mathrm{H}, J=14.2,8.0 \mathrm{~Hz}$ ), 2.03 (s, 3H), 1.00 (s, 9H). $\left.{ }^{13} \mathrm{C} \mathrm{NMR} \mathrm{(100} \mathrm{MHz,} \mathrm{CDCl}_{3}\right) \delta$ 170.0, 162.3, 144.0, 137.1, 135.6, 135.5, 134.6, 133.2, 133.0, 132.8, 129.8, 129.5, 129.0, 127.9, 127.8, 127.7, 127.5, 119.6, 96.6, 92.3, 89.1, 83.8, 79.9, 73.0, 62.1, 47.5, 26.8, 20.7, 19.2. FTIR (film) 3681, 1744, 1667, 1623, $1555 \mathrm{~cm}^{-1}$. HRMS (ESIMS) m/z calcd for $\mathrm{C}_{42} \mathrm{H}_{43} \mathrm{~N}_{3} \mathrm{O}_{7} \mathrm{SiBr}(\mathrm{M}+\mathrm{H})^{+}$808.2048, 810.2038, found 808.2097, 810.2011. (1'S,3'R,4'R,5'R,7'S)-(1'-m-Bromophenyl-4'-hydroxy-3'-hydroxymethyl-2',6'-diox abicyclo[3.3.0]oct-7'-yl)-thymine (mBr-WNA- $\beta$ T, 30b $(m))$. A white powder (86\%). Mp $192-194^{\circ} \mathrm{C}$ (dec.). $\quad{ }^{1} \mathrm{H}$ NMR (400 MHz, CD $\left.{ }_{3} \mathrm{OD}\right) \delta 7.95$ (bs, $\left.1 \mathrm{H}\right), 7.88$ (s, $\left.1 \mathrm{H}\right)$, 
7.71 (s, 1H), 7.69 (d, 1H, $J=7.9 \mathrm{~Hz}), 7.42$ (d, 1H, $J=7.9 \mathrm{~Hz}), 7.27$ (t, 1H, $J=7.9 \mathrm{~Hz}$ ), $6.25(\mathrm{t}, 1 \mathrm{H}, J=7.4 \mathrm{~Hz}), 4.83(\mathrm{~d}, 1 \mathrm{H}, J=3.6 \mathrm{~Hz}), 3.92-3.86(\mathrm{~m}, 1 \mathrm{H}), 3.69-3.65(\mathrm{~m}, 2 \mathrm{H})$, 3.67 (dd, $1 \mathrm{H}, J=12.2,5.6 \mathrm{~Hz}), 2.73(\mathrm{~d}, 2 \mathrm{H}, J=7.4 \mathrm{~Hz}), 1.93(\mathrm{~s}, 3 \mathrm{H}) .{ }^{13} \mathrm{C}$ NMR (100 $\left.\mathrm{MHz}, \mathrm{CD}_{3} \mathrm{OD}\right) \delta 165.6,150.8,142.5,139.1,131.7,130.9,129.9,125.5,123.5,111.7$, 92.6, 90.5, 84.7, 79.5, 73.4, 63.2, 34.3, 13.6. FTIR (film) 3362, 1693, 1674, $1580 \mathrm{~cm}^{-1}$. ESIMS (m/z) 439, $441(\mathrm{M}+\mathrm{H})^{+}$, HRMS (ESIMS) m/z calcd for $\mathrm{C}_{18} \mathrm{H}_{20} \mathrm{~N}_{2} \mathrm{O}_{6} \mathrm{Br}(\mathrm{M}+$ H) ${ }^{+}$439.0449, 441.0481, found 439.0449, 441.0495 .

(1'S,3'R,4'R,5'R,7'S)-(1'-o-Bromophenyl-4'-hydroxy-3'-hydroxymethyl-2',6'-dioxa bicyclo[3.3.0]oct-7'-yl)-thymine (oBr-WNA- $\beta$ T, 31b(o)). A colorless foam (99\%). ${ }^{1} \mathrm{H}$ NMR (500 MHz, CD 3 OD) $\delta 8.13$ (dd, 1H, $J=1.7,10.0 \mathrm{~Hz}$ ), 7.82 (d, 1H, $J=1.1 \mathrm{~Hz}$ ), 7.66-7.64 (m, 1H), 7.41-7.38 (m, 2H), 7.23-7.20 (m, 1H), 6.43 (dd, 1H, $J=8.8,5.8$ Hz), 5.43 (d, 1H, $J=3.8 \mathrm{~Hz}$ ), 4.01-3.98 (m, 1H), 3.92 (dd, 1H, $J=12.4,2.3 \mathrm{~Hz}$ ), 3.81 (dd, 1H, $J=9.3,4.1 \mathrm{~Hz}$ ), 3.68 (dd, $1 \mathrm{H}, J=12.4,5.5 \mathrm{~Hz}$ ), 3.06 (dd, $1 \mathrm{H}, J=13.7,8.8$ $\mathrm{Hz}$ ), 2.62 (dd, $1 \mathrm{H}, J=13.7,5.8 \mathrm{~Hz}), 1.93(\mathrm{~s}, 3 \mathrm{H}) .{ }^{13} \mathrm{C} \mathrm{NMR}\left(100 \mathrm{MHz}, \mathrm{CD}_{3} \mathrm{OD}\right) \delta$ 165.2, 152.3, 139.2, 137.1, 135.8, 131.0, 130.8, 129.0, 120.0, 112.0, 92.5, 88.1, 86.9, 84.3, 73.9, 63.1, 47.0, 12.6. FTIR (film) 3419, 1695, 1580, $1466 \mathrm{~cm}^{-1}$. HRMS 
(ESIMS) $\mathrm{m} / \mathrm{z}$ calcd for $\mathrm{C}_{18} \mathrm{H}_{19} \mathrm{~N}_{2} \mathrm{O}_{6} \mathrm{BrNa}(\mathrm{M}+\mathrm{Na})^{+}$461.0319, 463.0301, found 461.0317, 463.0297.

(1'S,3'R,4'R,5'R,7'S)-N $N^{4}$-Benzoyl-1-(1'-p-Bromophenyl-4'-hydroxy-3'-hydroxymet hyl-2',6'-dioxabicyclo[3.3.0]oct-7'-yl)-cytosine $\quad(p B r-W N A-\beta C, \quad 32 b(p)) . \quad$ A colorless form (79\%). ${ }^{1} \mathrm{H}$ NMR (400 MHz, $\left.\mathrm{CD}_{3} \mathrm{OD}\right) \delta 8.37$ (d, 1H, $J=7.6 \mathrm{~Hz}$ ), 7.98 (d, 2H, $J=7.3 \mathrm{~Hz}), 7.97-7.63$ (m, 4H), 7.56-7.49 (m, 4H), 6.34-6.30 (m, 1H), 4.91 (d, $1 \mathrm{H}, J=3.7 \mathrm{~Hz}$ ), 4.06-4.02 (m, 1H), 3.94-3.90 (m, 2H), 3.69 (dd, 1H, $J=12.0,5.5 \mathrm{~Hz}$ ), 3.00 (dd, $1 \mathrm{H}, J=14.0,6.0 \mathrm{~Hz}), 2.70(\mathrm{dd}, 1 \mathrm{H}, J=14.0,7.9 \mathrm{~Hz}) .{ }^{13} \mathrm{C}$ NMR $(100 \mathrm{MHz}$, $\left.\mathrm{CD}_{3} \mathrm{OD}\right) \delta 160.4,147.3,141.1,134.1,132.4,129.8,129.2,128.8,122.6,98.7,93.0$, 92.5, 91.4, 84.5, 73.4, 63.0, 50.1. FTIR (film) 3317, 1694, 1649, 1625, 1564, 1486 $\mathrm{cm}^{-1}$. HRMS (ESIMS) m/z calcd for $\mathrm{C}_{24} \mathrm{H}_{23} \mathrm{~N}_{3} \mathrm{O}_{6} \mathrm{Br}(\mathrm{M}+\mathrm{H})^{+}$528.0765, 530.0748, found 528.0759, 530.0714.

(1'S,3'R,4'R,5'R,7’S)-N $N^{4}$-Benzoyl-1-(1'-m-Bromophenyl-4'-hydroxy-3'-hydroxymet hyl-2',6'-dioxabicyclo[3.3.0]oct-7'-yl)-cytosine $\quad(m B r-W N A-\beta C, \quad 33 b(m)) . \quad$ A colorless form (82\%). $\quad{ }^{1} \mathrm{H}$ NMR (400 MHz, CD $\left.{ }_{3} \mathrm{OD}\right) \delta 8.39$ (d, $1 \mathrm{H}, J=7.3 \mathrm{~Hz}$ ), 7.99-7.94 (m, 3H), 7.69-7.61 (m, 3H), 7.56-7.52 (m, 2H), 7.44-7.41 (m, 1H), 
7.29-7.25 (m, 1H), 6.31 (dd, 1H, $J=6.1,7.9 \mathrm{~Hz}$ ), 4.93 (d, 1H, $J=8.2 \mathrm{~Hz}$ ), 4.07-4.03 (m, 1H), 3.94-3.90 (m, 2H), 3.70 (dd, 1H, $J=12.2,5.5 \mathrm{~Hz}$ ), 2.98 (dd, 1H, $J=14.0,6.1$ $\mathrm{Hz}), 2.73(\mathrm{dd}, 1 \mathrm{H}, J=14.0,7.9 \mathrm{~Hz}) .{ }^{13} \mathrm{C} \mathrm{NMR}\left(100 \mathrm{MHz}, \mathrm{CD}_{3} \mathrm{OD}\right) \delta 160.1,147.4$, 144.39 134.7, 134.1, 131.2, 129.8, 129.2, 125.5, 123.6, 98.7, 92.8, 92.7, 91.5, 84.4, 73.3, 63.1, 50.1. FTIR (film) 3383, 1697, 1652, 1565, $1505 \mathrm{~cm}^{-1}$. HRMS (ESIMS) m/z calcd for $\mathrm{C}_{24} \mathrm{H}_{23} \mathrm{~N}_{3} \mathrm{O}_{6} \mathrm{Br}(\mathrm{M}+\mathrm{H})^{+}$528.0765, 530.0748, found 528.0791, 530.0783.

(1'S,3'R,4'R,5'R,7'S)-N $N^{4}$-Benzoyl-1-(1'-o-Bromophenyl-4'-hydroxy-3'-hydroxymet hyl-2',6'-dioxabicyclo[3.3.0]oct-7'-yl)-cytosine $\quad($ oBr-WNA- $\beta C, \quad 34 b(o)) . \quad$ A colorless form (78\%). $\quad{ }^{1} \mathrm{H}$ NMR (400 MHz, CD $\left.{ }_{3} \mathrm{OD}\right) \delta 8.53(\mathrm{~d}, 1 \mathrm{H}, J=7.6 \mathrm{~Hz}$ ), 8.12-8.10 (m, 1H), 7.98-7.95 (m, 2H), 7.70-7.50 (m, 5H), 7.40-7.17 (m, 2H), 6.43-6.40 (m, 1H), 5.50 (d, 1H, $J=4.0 \mathrm{~Hz}$ ), 4.07-4.04 (m, 1H), 3.95 (dd, 1H, $J=12.2$, $2.1 \mathrm{~Hz}$ ), 3.84 (dd, $1 \mathrm{H}, J=10.4,4.0 \mathrm{~Hz}$ ), 3.71 (dd, $1 \mathrm{H}, J=12.2,5.3 \mathrm{~Hz}$ ), 3.01 (dd, $1 \mathrm{H}, J$ $=14.4,7.6 \mathrm{~Hz}), 2.94(\mathrm{dd}, 1 \mathrm{H}, J=14.4,6.1 \mathrm{~Hz}) .{ }^{13} \mathrm{C} \mathrm{NMR}\left(100 \mathrm{MHz}, \mathrm{CD}_{3} \mathrm{OD}\right) \delta$ 169.1, 164.9, 157.7, 145.6, 140.0, 135.8, 134.7, 134.1, 131.0, 129.8, 129.2, 129.0, 120.7, 98.6, 92.7, 90.8, 88.1, 83.8, 73.7, 62.9, 49.8. FTIR (film) 3307, 1695, 1653, 1622, 
1562, $1486 \mathrm{~cm}^{-1}$. HRMS (ESIMS) m/z calcd for $\mathrm{C}_{24} \mathrm{H}_{23} \mathrm{~N}_{3} \mathrm{O}_{6} \mathrm{Br}(\mathrm{M}+\mathrm{H})^{+}$528.0765, 530.0748, found 528.0718, 530.0756.

(1'S,3'R,4'R,5'R,7'S)-\{1'-m-Bromophenyl-3'-dimethoxytrithyloxymethyl-4'-O-( $N$, $N$-diisopropyl- $\beta$-cyanoethylphosphoramidyl)-2',6'-dioxabicyclo[3.3.0]oct-7'-yl\}-thy mine (mBr-WNA- $\boldsymbol{\beta T}, 30 \mathbf{c}(\boldsymbol{m}))$. A white powder $(53 \%) . \quad{ }^{1} \mathrm{H}$ NMR (400 MHz, $\left.\mathrm{CDCl}_{3} / \mathrm{TMS}\right) \delta 8.71$ (bs, $\left.1 \mathrm{H}\right), 7.80$ (d, $1 \mathrm{H}, J=6.9 \mathrm{~Hz}$ ), 7.62 (d, 0.5H, $\left.J=7.9 \mathrm{~Hz}\right), 7.56$ (d, 0.5H, $J=7.9 \mathrm{~Hz}$ ), 7.46-7.42 (m, 2H), 7.35-7.18 (m, 10H), 6.83 (d, 4H, $J=8.8 \mathrm{~Hz}$ ), $6.37(\mathrm{t}, 0.5 \mathrm{H}, J=7.1 \mathrm{~Hz}), 6.17(\mathrm{t}, 0.5 \mathrm{H}, J=7.0 \mathrm{~Hz}), 5.07$ (d, 0.5H, $J=3.4 \mathrm{~Hz}), 4.97$ (d, 0.5H, $J=3.2 \mathrm{~Hz}), 4.39-4.34(\mathrm{~m}, 0.5 \mathrm{H}), 4.30-4.24(\mathrm{~m}, 1 \mathrm{H}), 4.17-4.12(\mathrm{~m}, 0.5 \mathrm{H}), 3.81$ (s, 3H), $3.80(\mathrm{~s}, 3 \mathrm{H}), 3.77-3.71(\mathrm{~m}, 2 \mathrm{H}), 3.61-3.40(\mathrm{~m}, 4 \mathrm{H}), 3.25-3.22(\mathrm{~m}, 1 \mathrm{H})$, 3.00-2.94 (m, 1H), 2.59 (dd, 1H, $J=12.0,5.8 \mathrm{~Hz}$ ), 2.37 (dd, 1H, $J=12.0,6.7 \mathrm{~Hz}$ ), 2.01 (s, 1.5H), 1.98 (s, 1.5H), 1.15 (d, 4H, $J=6.7 \mathrm{~Hz}), 1.09$ (d, 6H, $J=6.7 \mathrm{~Hz}$ ), 0.93 (d, 2H, $J=6.7 \mathrm{~Hz}) . \quad{ }^{31} \mathrm{P}$ NMR $\left(161.9 \mathrm{MHz}, \mathrm{CDCl}_{3}\right) \delta 149.9,149.2 . \quad$ FTIR (film) 2252, 1693, 1681, 1666, 1564, $1486 \mathrm{~cm}^{-1} . \quad \operatorname{ESIMS}(\mathrm{m} / \mathrm{z}) 941,943(\mathrm{M}+\mathrm{H})^{+}$.

(1'S,3'R,4'R,5'R,7'S)-\{1'-o-Bromophenyl-3'-dimethoxytrithyloxymethyl-4'-O-( $N, N$ -diisopropyl- $\beta$-cyanoethylphosphoramidyl)-2',6'-dioxabicyclo[3.3.0]oct-7'-yl\}-thym 
ine (oBr-WNA- $\beta$ T, 31c(o)). A white powder $(66 \%) . \quad{ }^{1} \mathrm{H}$ NMR $(400 \mathrm{MHz}$, $\left.\mathrm{CDCl}_{3} / \mathrm{TMS}\right) \delta$ 8.09-8.00 (m, 2H), 7.71-7.60 (m, 2H), 7.45-7.41 (m, 2H), 7.34-7.27 (m, 4H), 7.25-7.13 (m, 4H), 6.79 (d, 4H, $J=8.8 \mathrm{~Hz}$ ), 6.51 (dd, 0.5H, $J=8.4,5.7 \mathrm{~Hz}$ ), 6.44 (dd, 0.5H, $J=8.4,5.9 \mathrm{~Hz}), 5.49(\mathrm{~d}, 1 \mathrm{H}, J=4.5 \mathrm{~Hz}), 4.28-4.20(\mathrm{~m}, 1.5 \mathrm{H})$, 3.99-3.97 (m, 0.5H), 3.78 (s, 3H), 3.77 (s, 3H), 3.72-3.38 (m, 5H), 3.21-3.18 (m, 1H), 2.99-2.93 (m, 1H), 2.83-2.77 (m, 1H), 2.58-2.55 (m, 1H), 2.38-2.30 (m, 1H), 1.96 (s, 1.5H), 1.93 (s, 1.5H), 1.13 (d, 3H, $J=6.7 \mathrm{~Hz}), 1.07$ (t, 6H, $J=6.6 \mathrm{~Hz}), 0.87$ (d, 3H, $J=$

6.7 Hz). ${ }^{31} \mathrm{P}$ NMR (161.9 MHz, $\left.\mathrm{CDCl}_{3}\right) \delta$ 150.4, 149.3. FTIR (film) 2359, 1700, 1681, 1608, 15609, $1464 \mathrm{~cm}^{-1} . \quad \operatorname{ESIMS}(\mathrm{m} / \mathrm{z})$ 963, $965(\mathrm{M}+\mathrm{Na})^{+}$.

(1'S,3'R,4'R,5'R,7’S)-N $N^{4}$-Benzoyl-1-\{1'-p-Bromophenyl-3'-dimethoxytrithyloxymet hyl-4'-O-( $N, N$-diisopropyl- $\beta$-cyanoethylphosphoramidyl)-2',6'-dioxabicyclo[3.3.0]o ct-7'-yl\}-cytosine (pBr-WNA- $\beta$ C, 32c(p)). A white powder (66\%). $\quad{ }^{1} \mathrm{H}$ NMR (400 $\left.\mathrm{MHz}, \mathrm{CDCl}_{3} / \mathrm{TMS}\right) \delta 8.76$ (bs, $1 \mathrm{H}$ ), 8.13 (d, 0.5H, $J=7.3 \mathrm{~Hz}$ ), 7.90 (d, 2.5H, $J=7.3$ Hz), 7.88-7.37 (m, 10H), 7.31-7.22 (m, 7H), 6.81-6.79 (m, 4H), 6.36-6.33 (m, 0.5H), 6.22-6.19 (m, 0.5H), 5.04 (d, 0.5H, $J=2.8 \mathrm{~Hz}$ ), 4.97 (d, 0.5H, $J=2.8 \mathrm{~Hz}$ ), 4.36-4.16 (m, 2H), 3.79 (s, 3H), 3.78 (s, 3H), 3.71-3.15 (m, 7H), 2.64-2.29 (m, 3H), 1.41-1.24 (m, 
3H), 1.23-1.06 (m, 5H), 0.95-0.85 (m, 4H). ${ }^{31} \mathrm{P}$ NMR (161.9 MHz, $\left.\mathrm{CDCl}_{3}\right) \delta 150.2$, 149.5. FTIR (film) 2359, 1696, 1664, 1624, 1609, 1555, $1508 \mathrm{~cm}^{-1}$. (1'S,3'R,4'R,5'R,7’S)- $N^{4}$-Benzoyl-1-\{1'-m-Bromophenyl-3'-dimethoxytrithyloxyme thyl-4'-O-( $N, N$-diisopropyl- $\beta$-cyanoethylphosphoramidyl)-2',6'-dioxabicyclo[3.3.0] oct-7'-yl\}-cytosine (mBr-WNA- $\boldsymbol{\beta C}, 33 \mathbf{3}(\boldsymbol{m})) . \quad$ A white powder $(36 \%) .{ }^{1} \mathrm{H}$ NMR (400 MHz, $\mathrm{CDCl}_{3} / \mathrm{TMS}$ ) $\delta 8.73$ (bs, 1H), 8.14 (d, 0.5H, $J=7.3 \mathrm{~Hz}$ ), 7.94 (d, 0.5H, $J=$ $7.3 \mathrm{~Hz}$ ), 7.89 (d, 2H, $J=8.2 \mathrm{~Hz}), 7.75$ (d, 1H, $J=11.6 \mathrm{~Hz}), 7.62-7.40(\mathrm{~m}, 8 \mathrm{H})$, 7.38-7.13 (m, 8H), 6.82-6.79 (m, 4H), 6.39-6.36 (m, 0.5H), 6.26-6.23 (m, 0.5H), 5.07 (d, 0.5H, $J=3.7 \mathrm{~Hz}$ ), 5.00 (d, 0.5H, $J=3.7 \mathrm{~Hz}$ ), 4.39-4.11 (m, 2H), 3.78 (s, 3H), 3.77 (s, 3H), 3.73-3.19 (m, 7H), 2.58-2.26 (m, 3H), 1.35-1.31 (m, 3H), 1.20-1.06 (m, 5H), 0.95-0.85 (m, 4H). ${ }^{31} \mathrm{P}$ NMR (161.9 MHz, $\left.\mathrm{CDCl}_{3}\right) \delta 150.2,149.4 . \quad$ FTIR (film) 2359, 1666, 1608, 1562, $1508 \mathrm{~cm}^{-1}$.

(1'S,3'R,4'R,5'R,7'S)-N $N^{4}$-Benzoyl-1-\{1'-o-Bromophenyl-3'-dimethoxytrithyloxymet hyl-4'-O-( $N, N$-diisopropyl- $\beta$-cyanoethylphosphoramidyl)-2',6'-dioxabicyclo[3.3.0]o ct-7'-yl\}-cytosine (oBr-WNA- $\beta$ C, $34 \mathbf{3}(\boldsymbol{o})) . \quad$ A white powder (78\%). $\quad{ }^{1} \mathrm{H}$ NMR (400 $\left.\mathrm{MHz}, \mathrm{CDCl}_{3} / \mathrm{TMS}\right) \delta 8.70$ (bs, $\left.1 \mathrm{H}\right), 8.41$ (d, 0.5H, $J=7.3 \mathrm{~Hz}$ ), 8.27 (d, 2.5H, $J=7.3$ 
$\mathrm{Hz}$ ), $8.11(\mathrm{~d}, 0.5 \mathrm{H}, J=7.6 \mathrm{~Hz}), 8.03$ (d, 0.5H, $J=7.6 \mathrm{~Hz}), 7.89$ (d, 2H, $J=7.3 \mathrm{~Hz}$ ), 7.62-7.44 (m, 8H), 7.34 (d, 4H, $J=8.9 \mathrm{~Hz}), 7.28-7.11(\mathrm{~m}, 4 \mathrm{H}), 6.82(\mathrm{~d}, 2 \mathrm{H}, J=8.8$ Hz), 6.81 (d, 2H, J =8.9 Hz), 6.52-6.46 (m, 1H), 5.57-5.54 (m, 1H), 4.28-4.16 (m, 1.5H), 4.00-3.95 (m, 0.5H), 3.79 (s, 3H), 3.78 (s, 3H), 3.77-2.92 (m, 8H), 2.58-2.55 (m, 1H), 2.39-2.28 (m, 1H), 1.28-1.20 (m, 3H), 1.15-1.07 (m, 5H), 0.95-0.85 (m, 4H). ${ }^{31} \mathrm{P}$ NMR (161.9 MHz, $\left.\mathrm{CDCl}_{3}\right) \delta$ 150.7, 149.5. FTIR (film) 2359, 1696, 1665, 1609, $1557,1508 \mathrm{~cm}^{-1}$. 

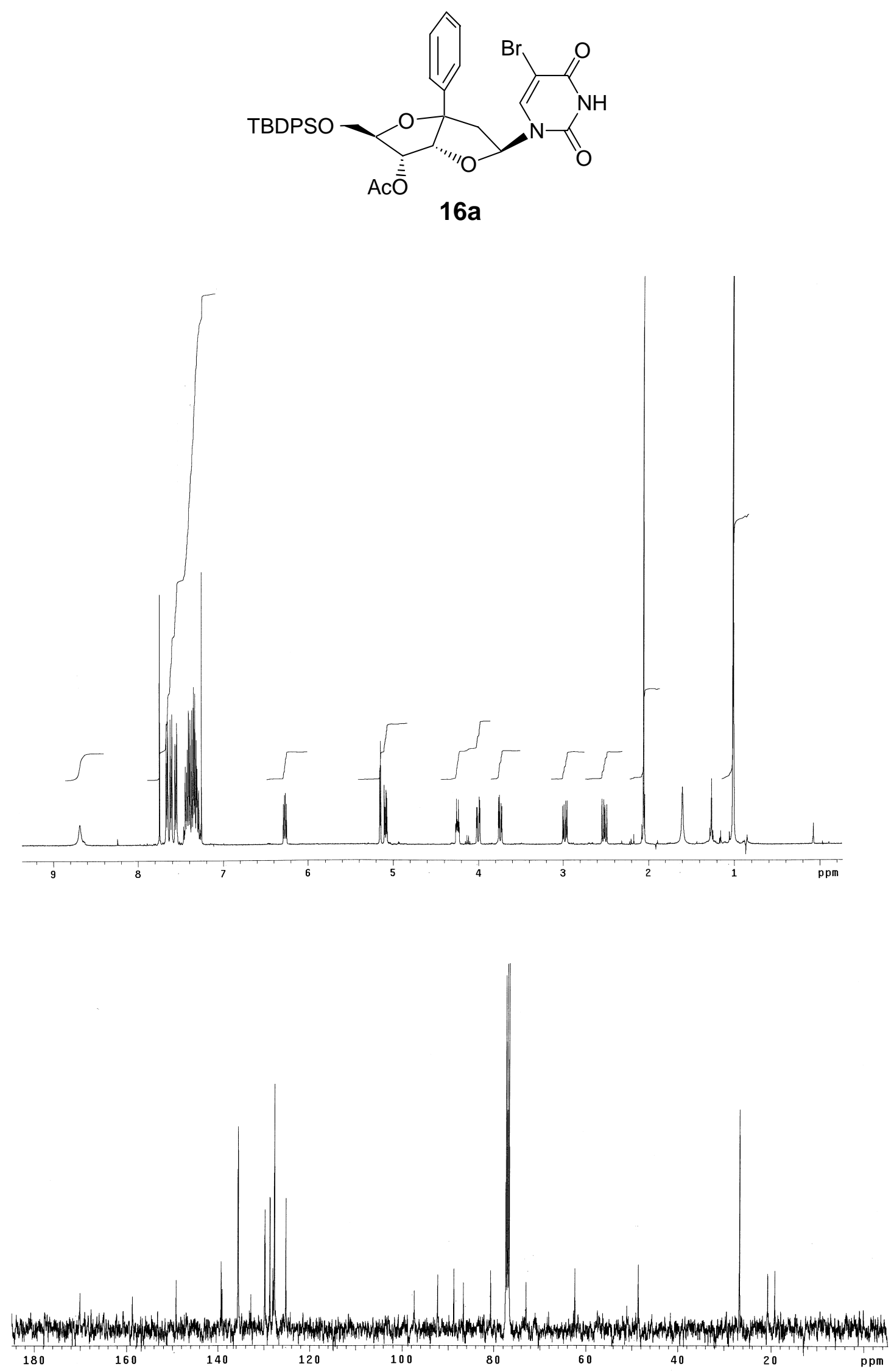

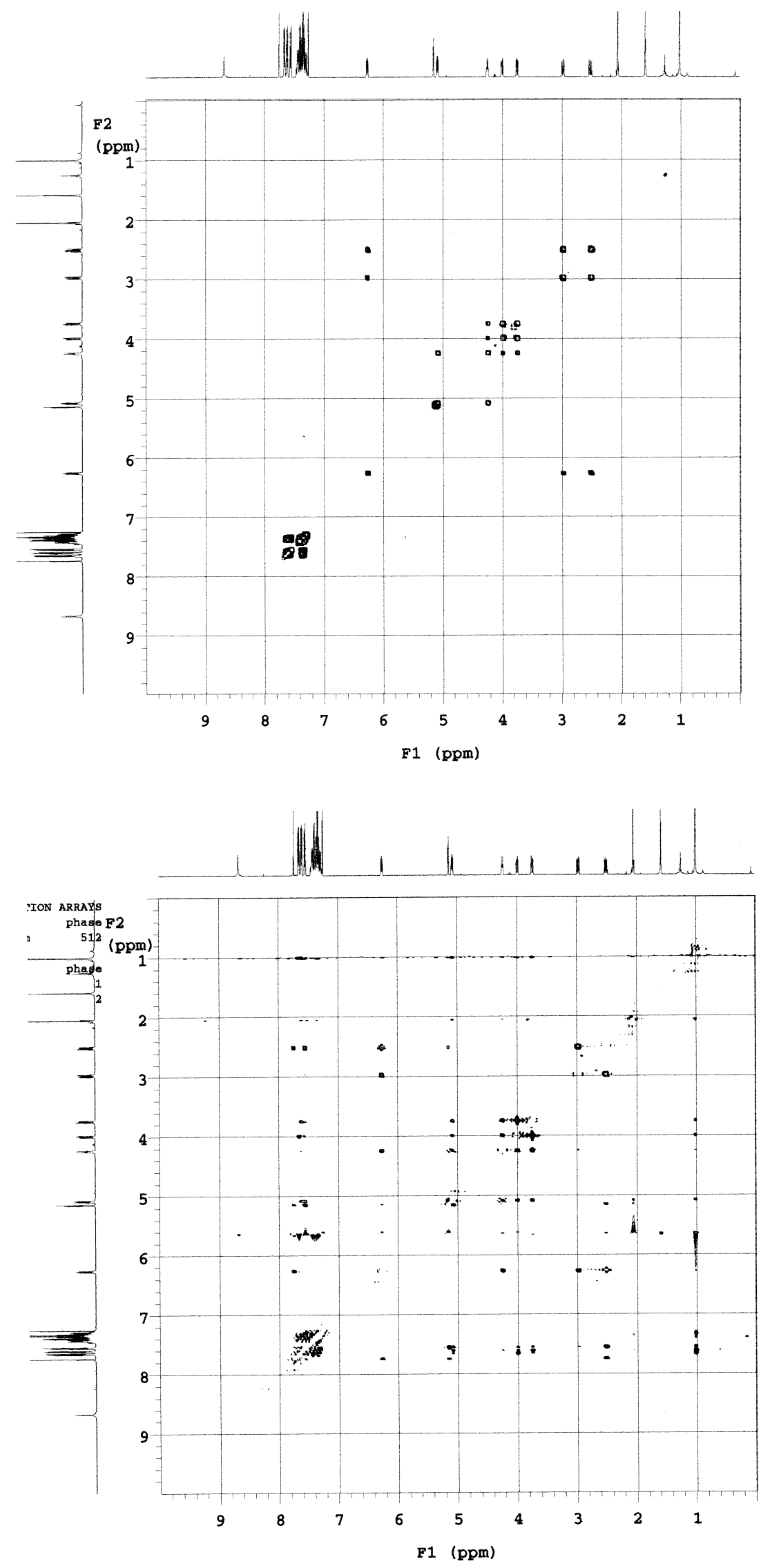

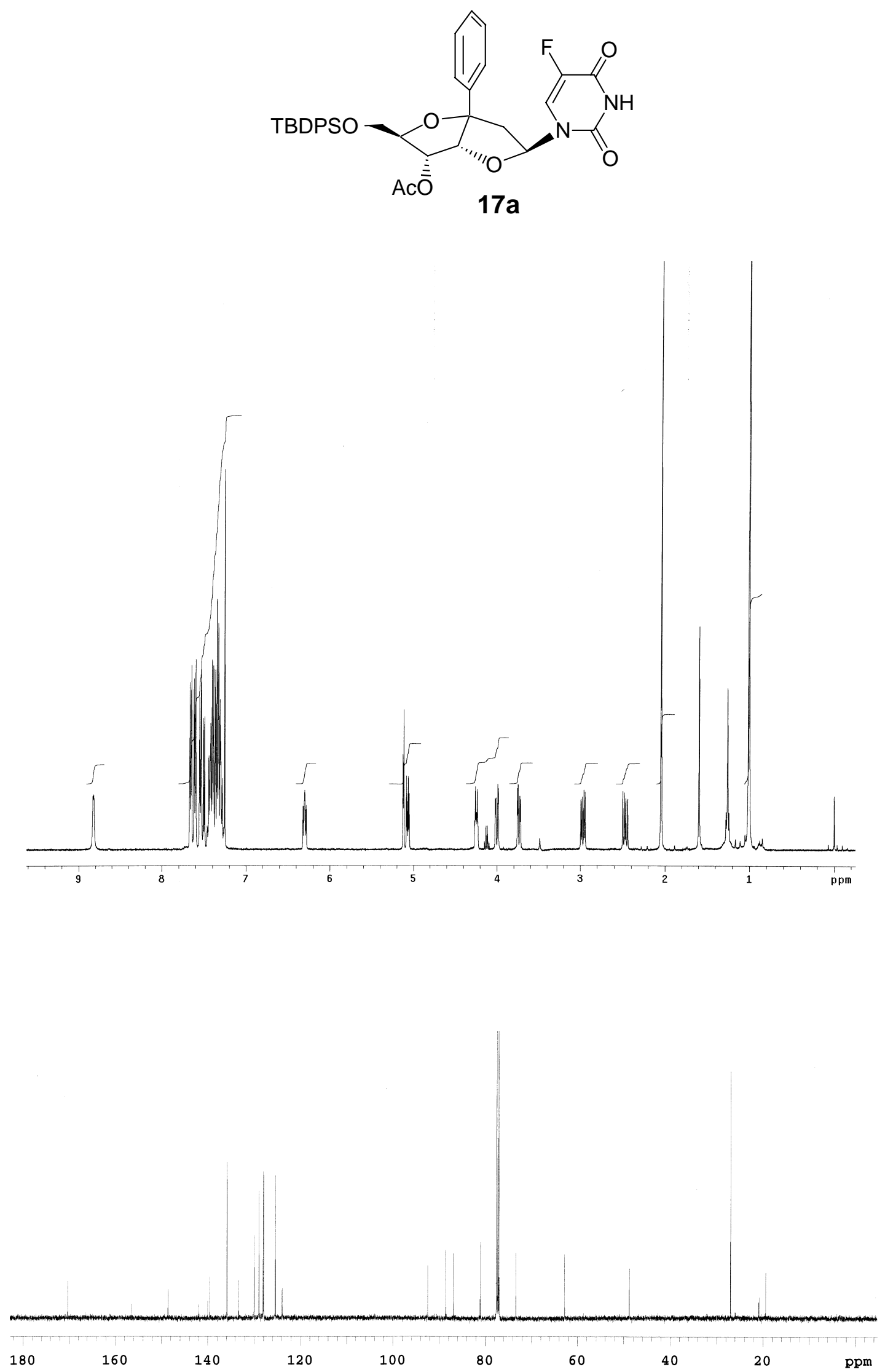

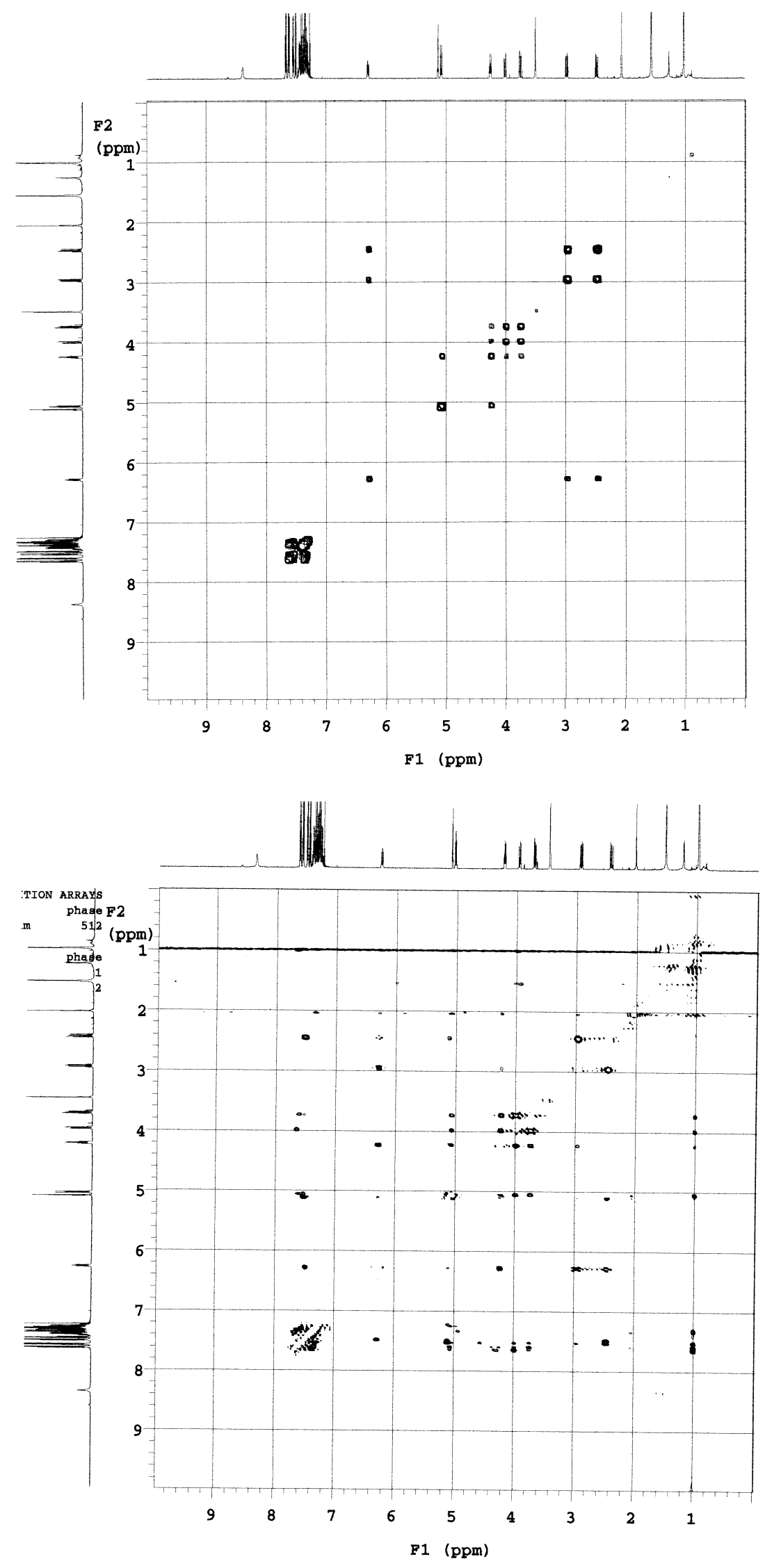

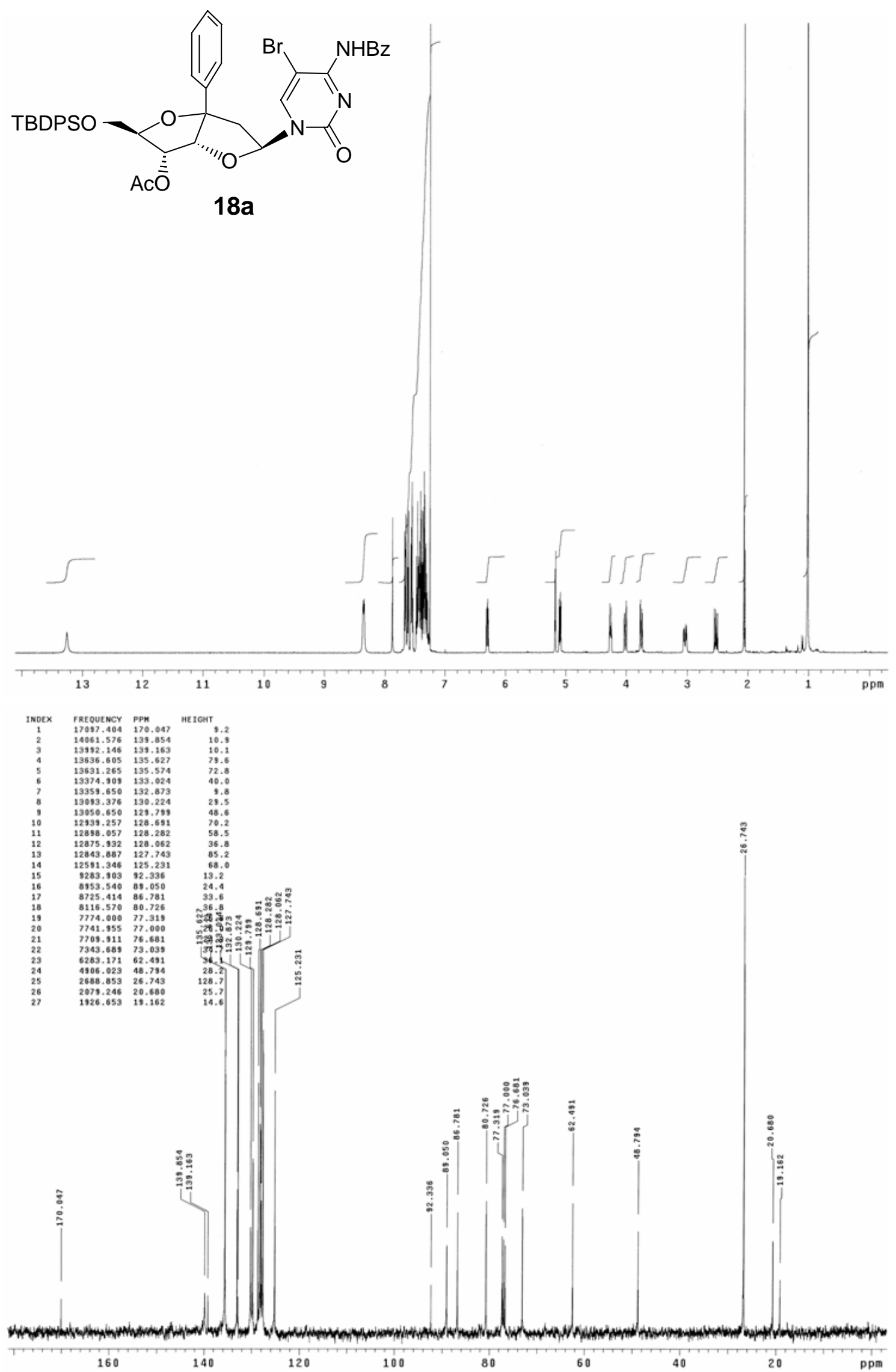

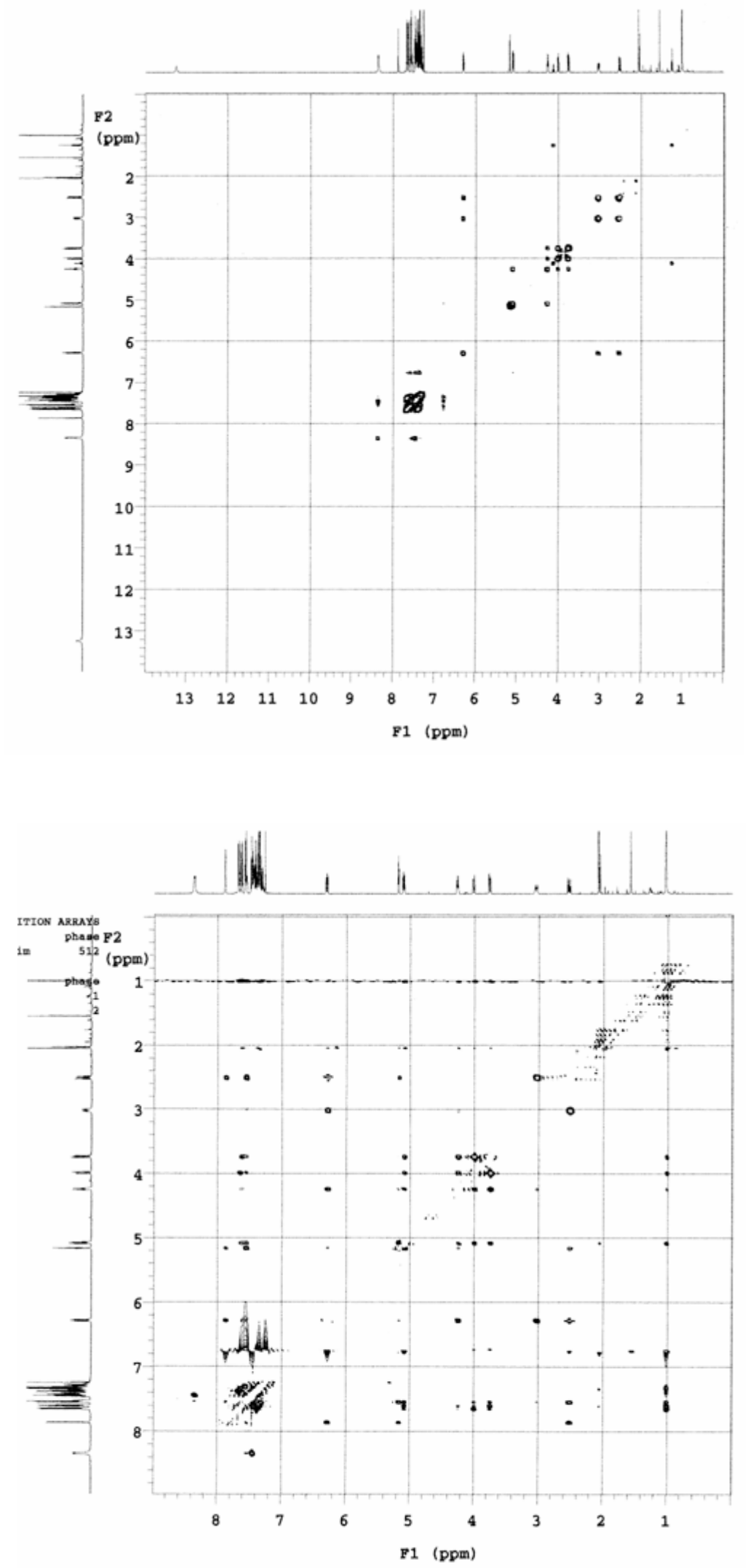

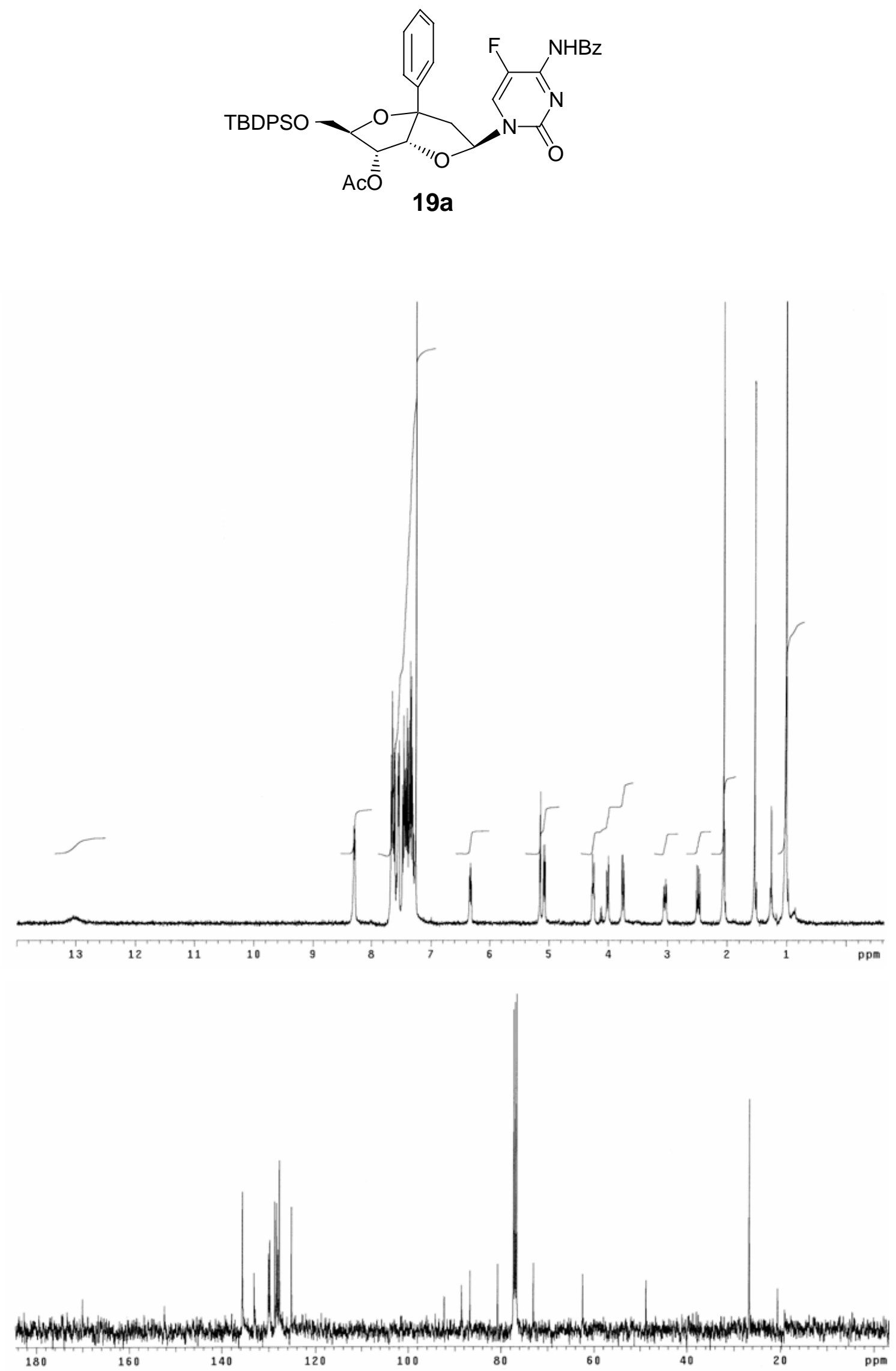

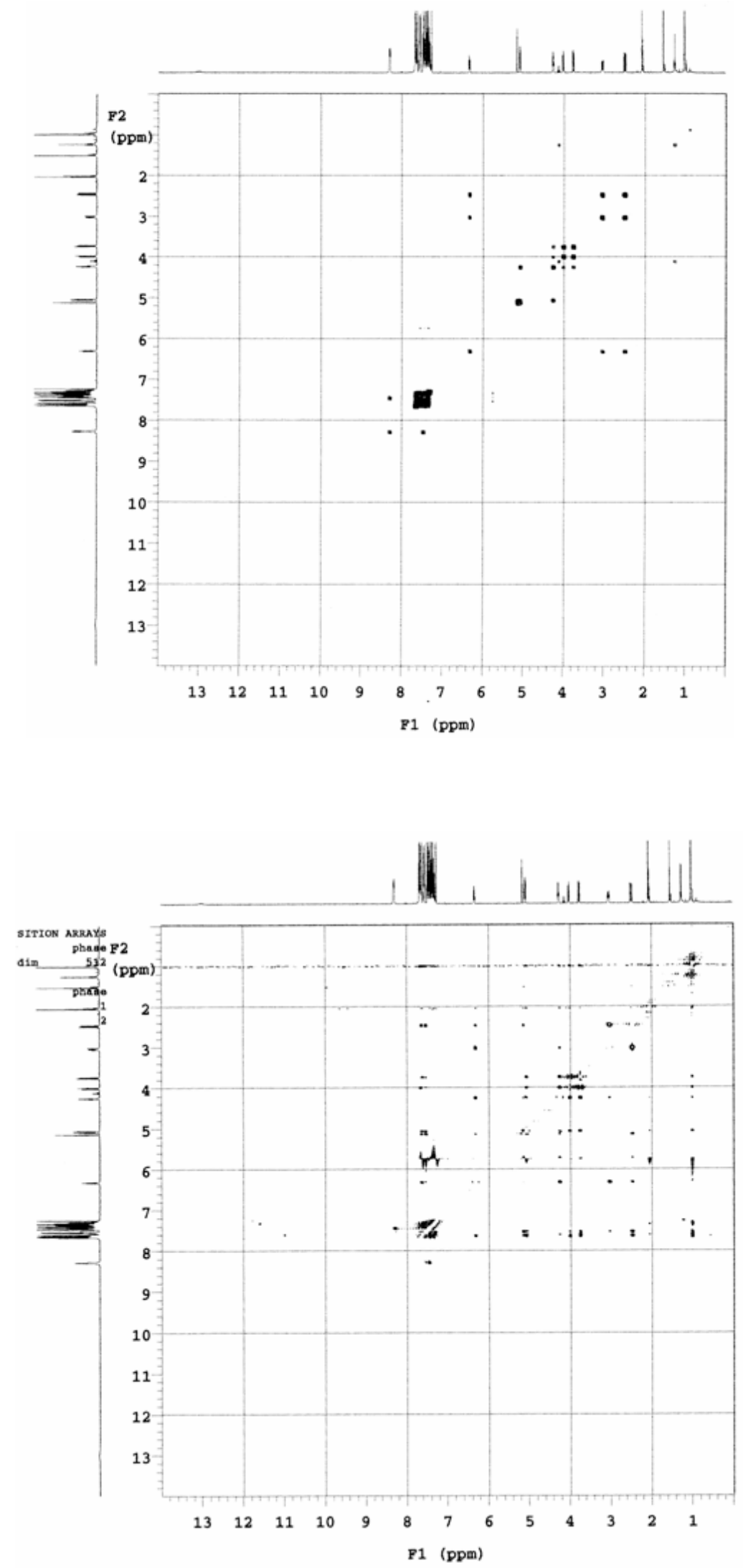

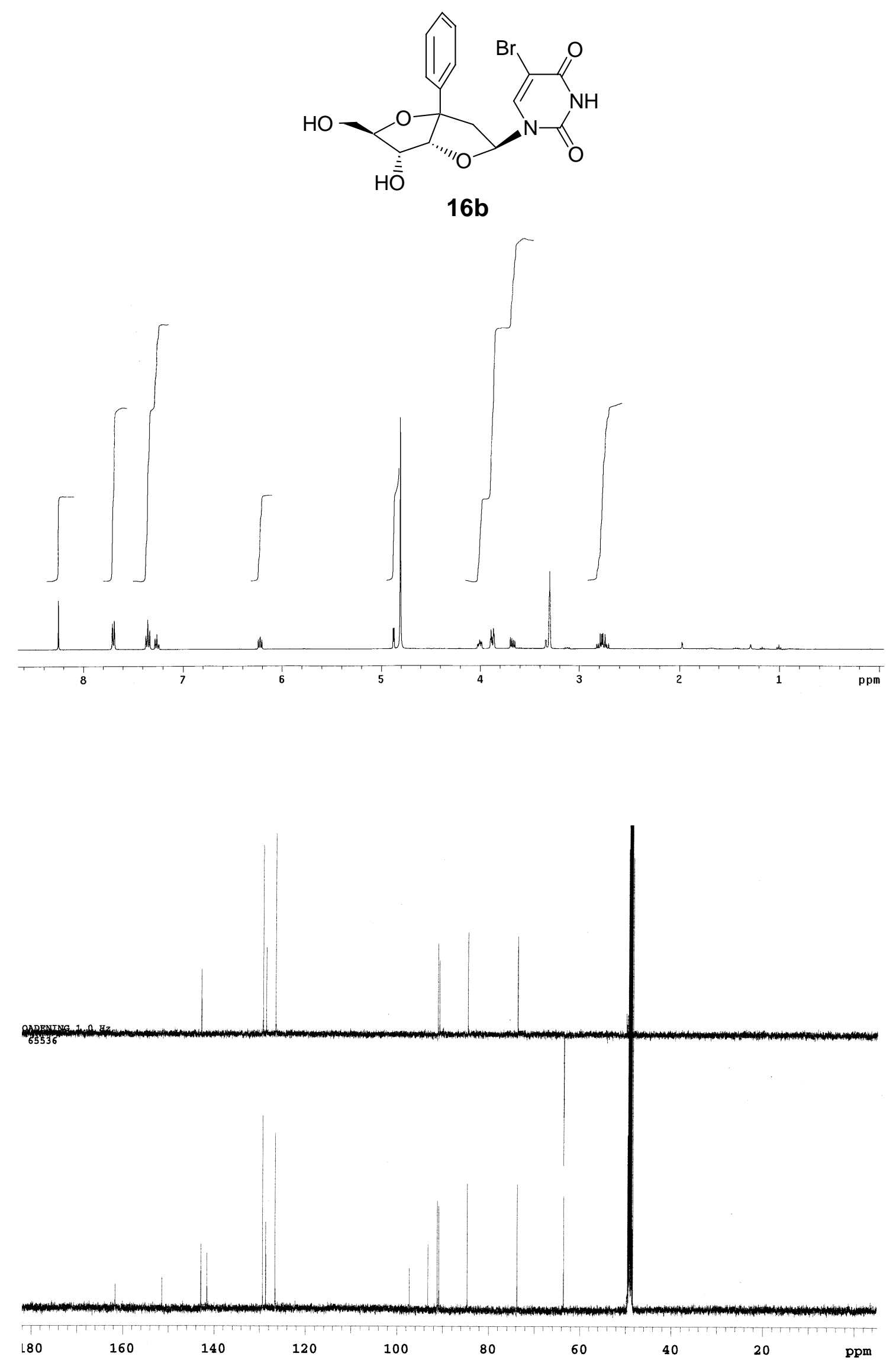


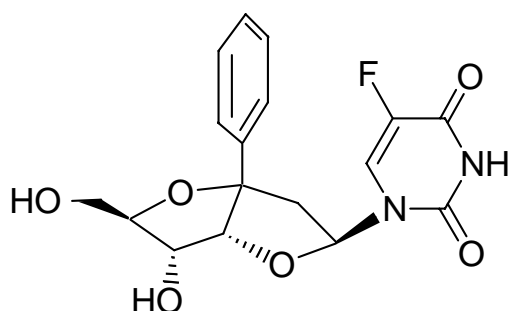

17b
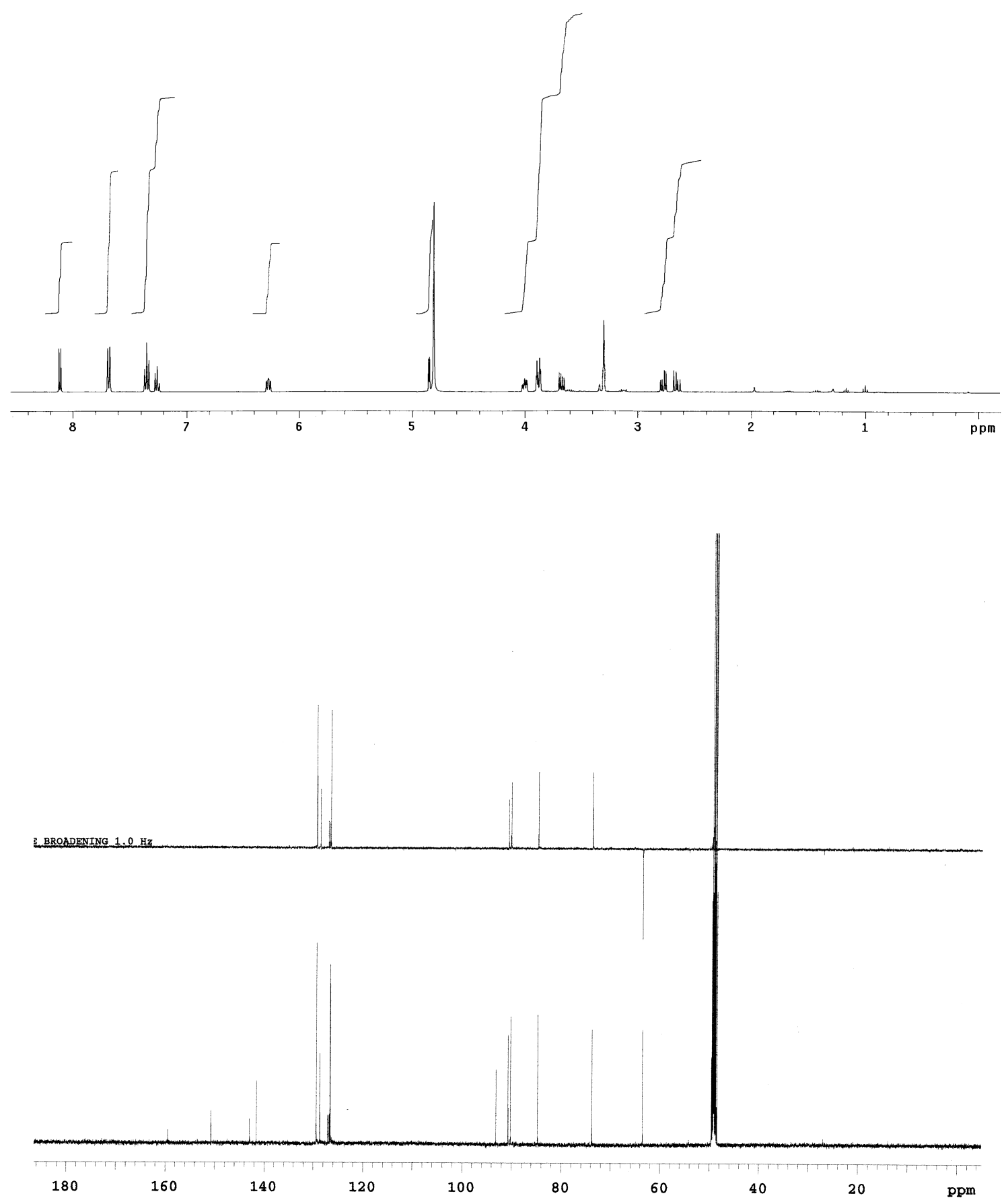

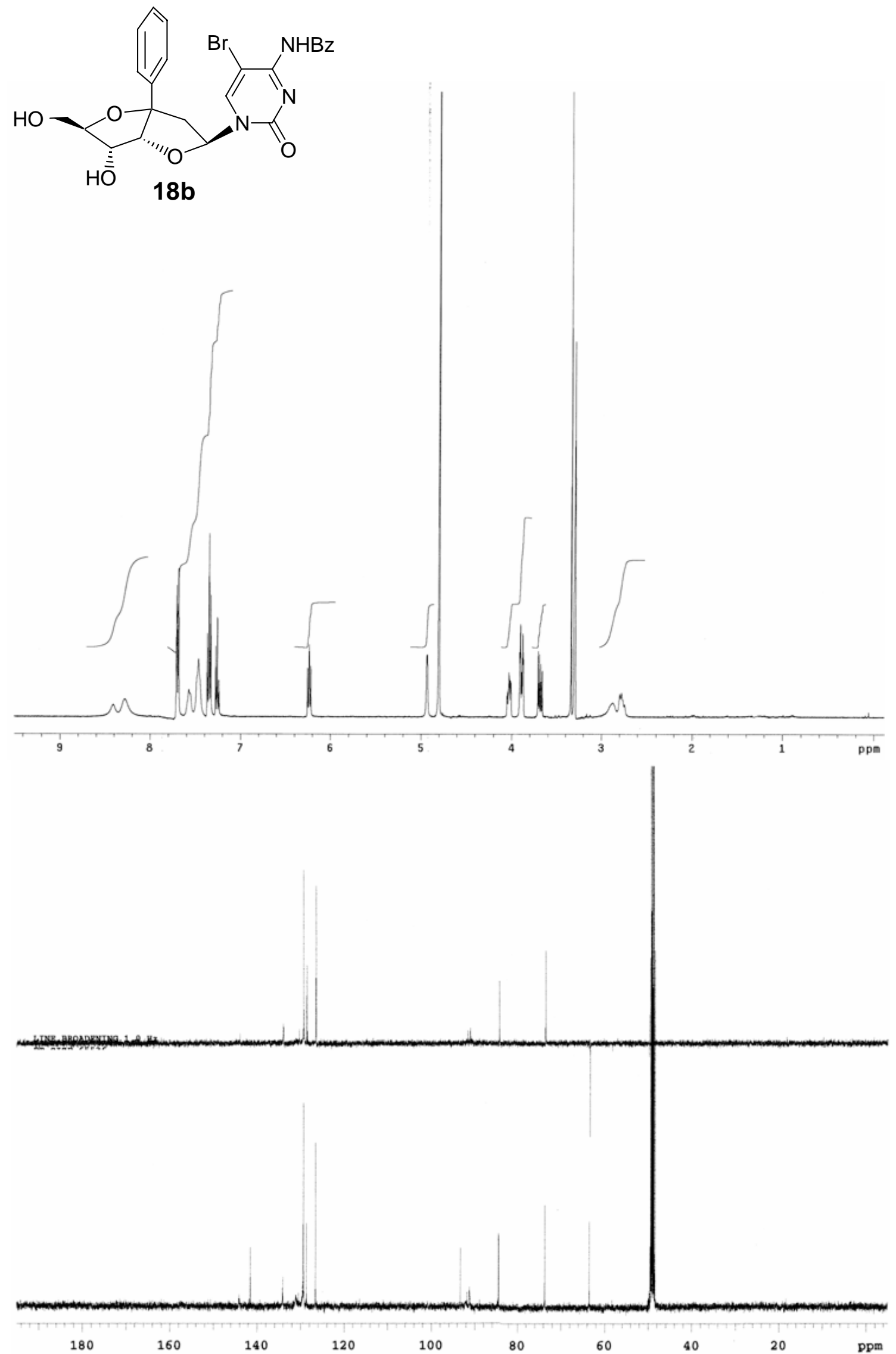

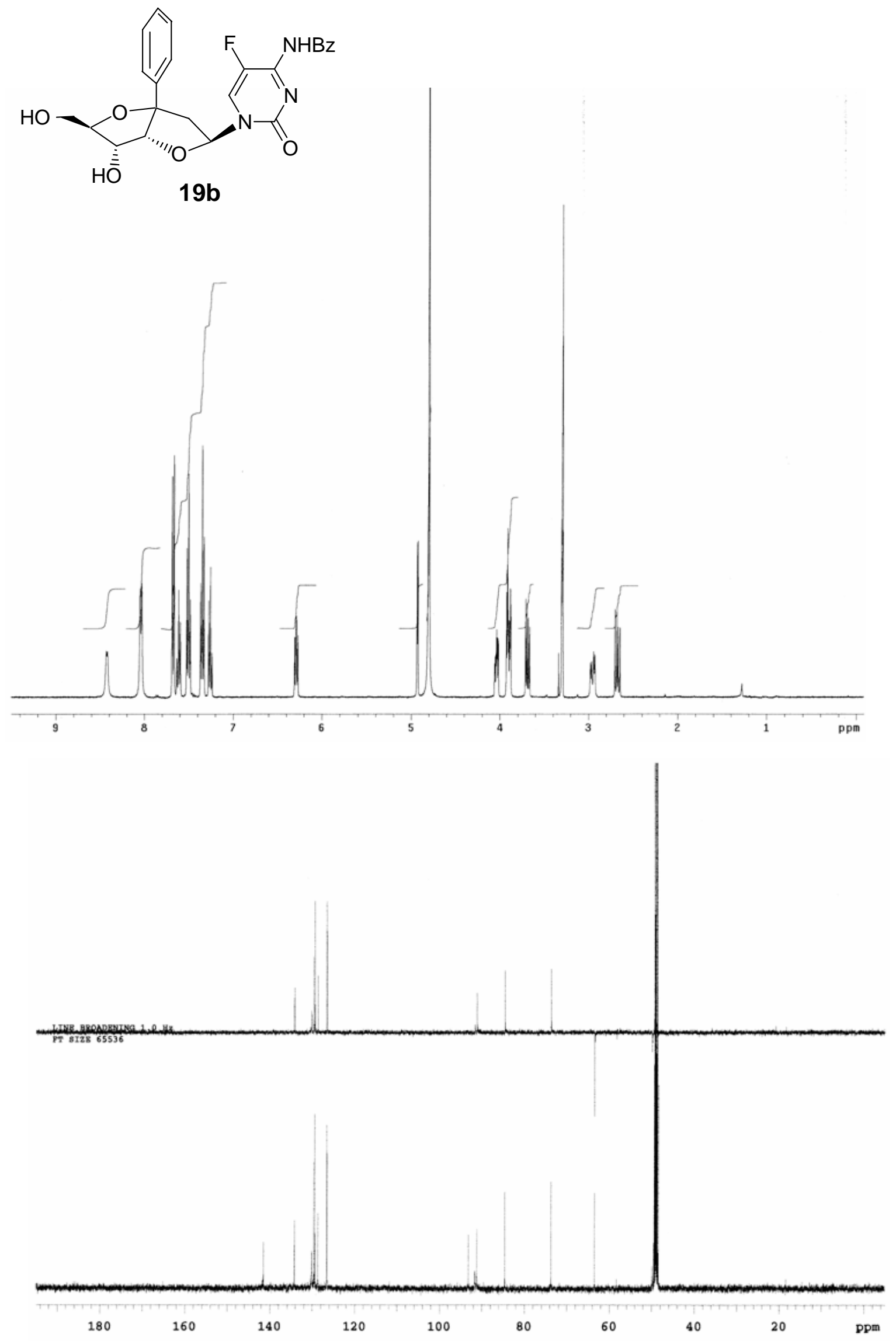

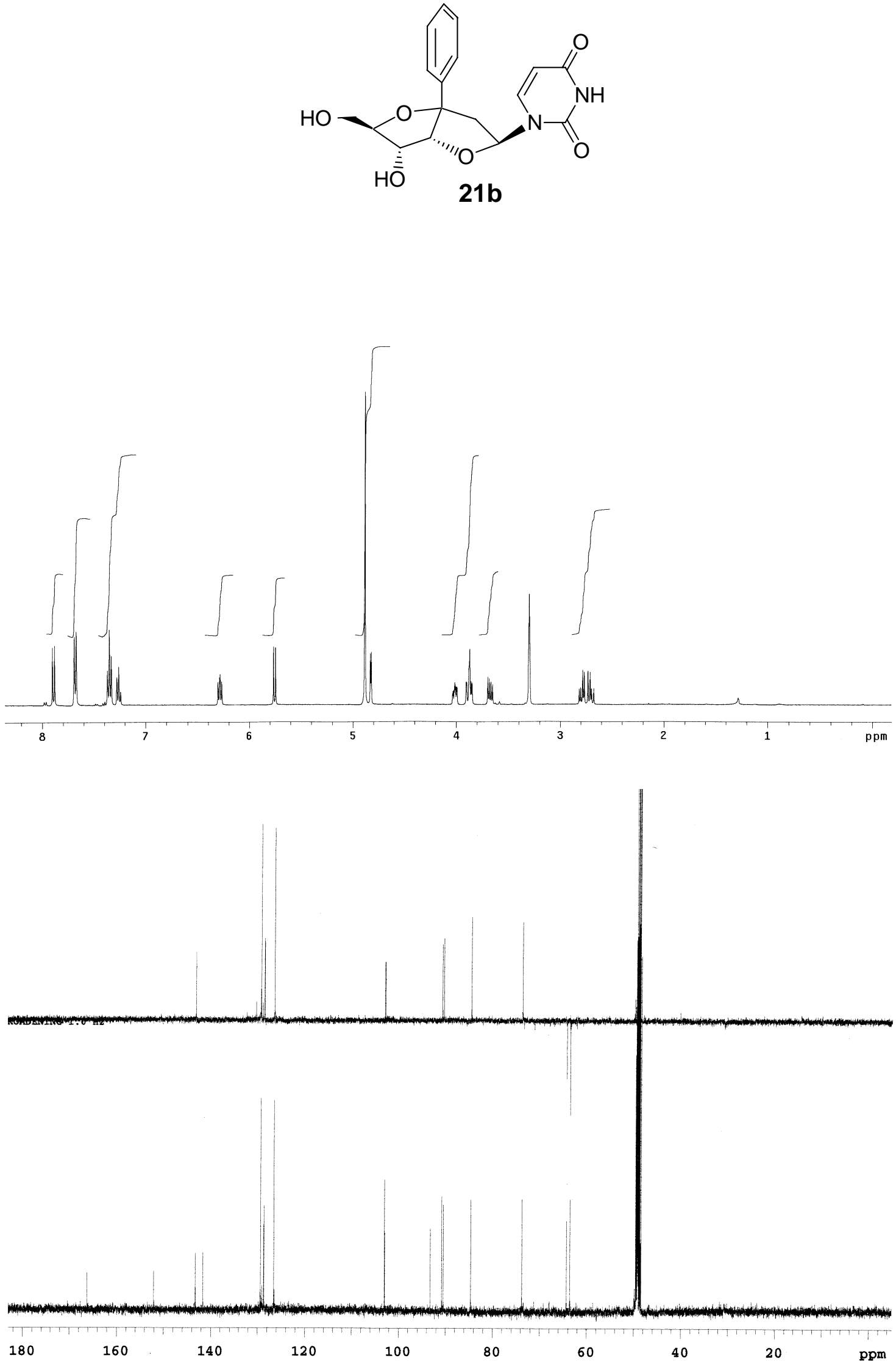

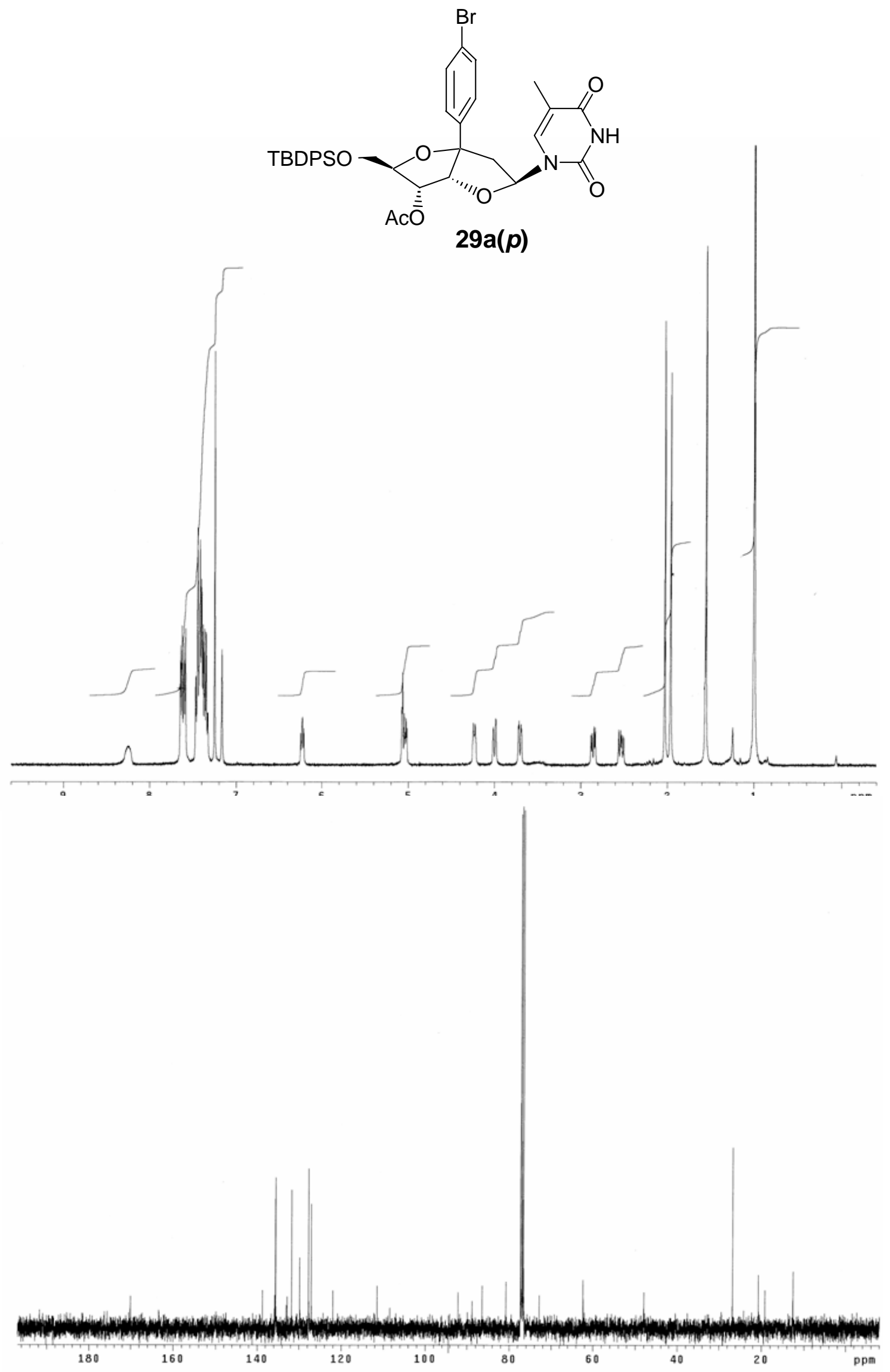

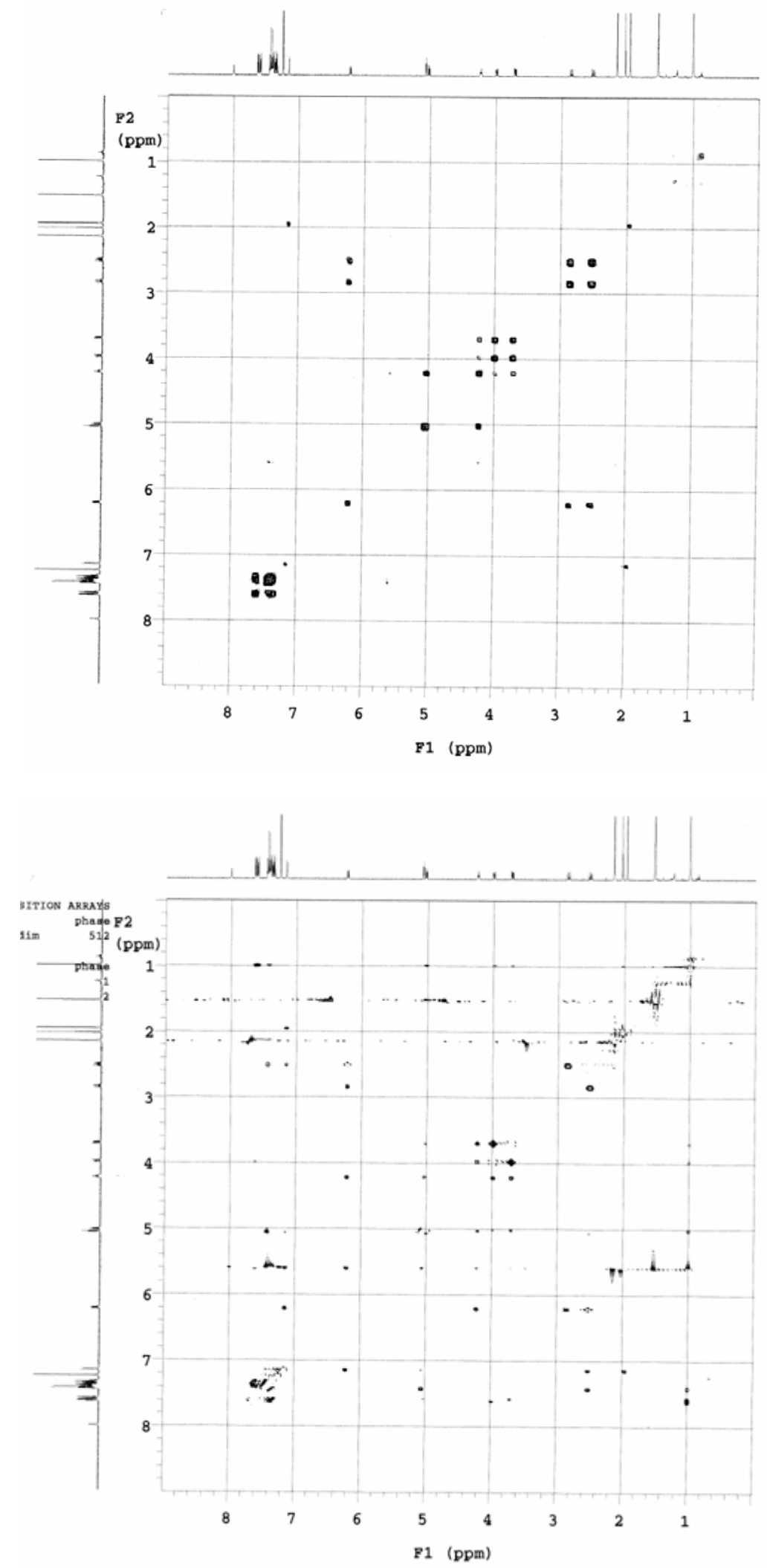

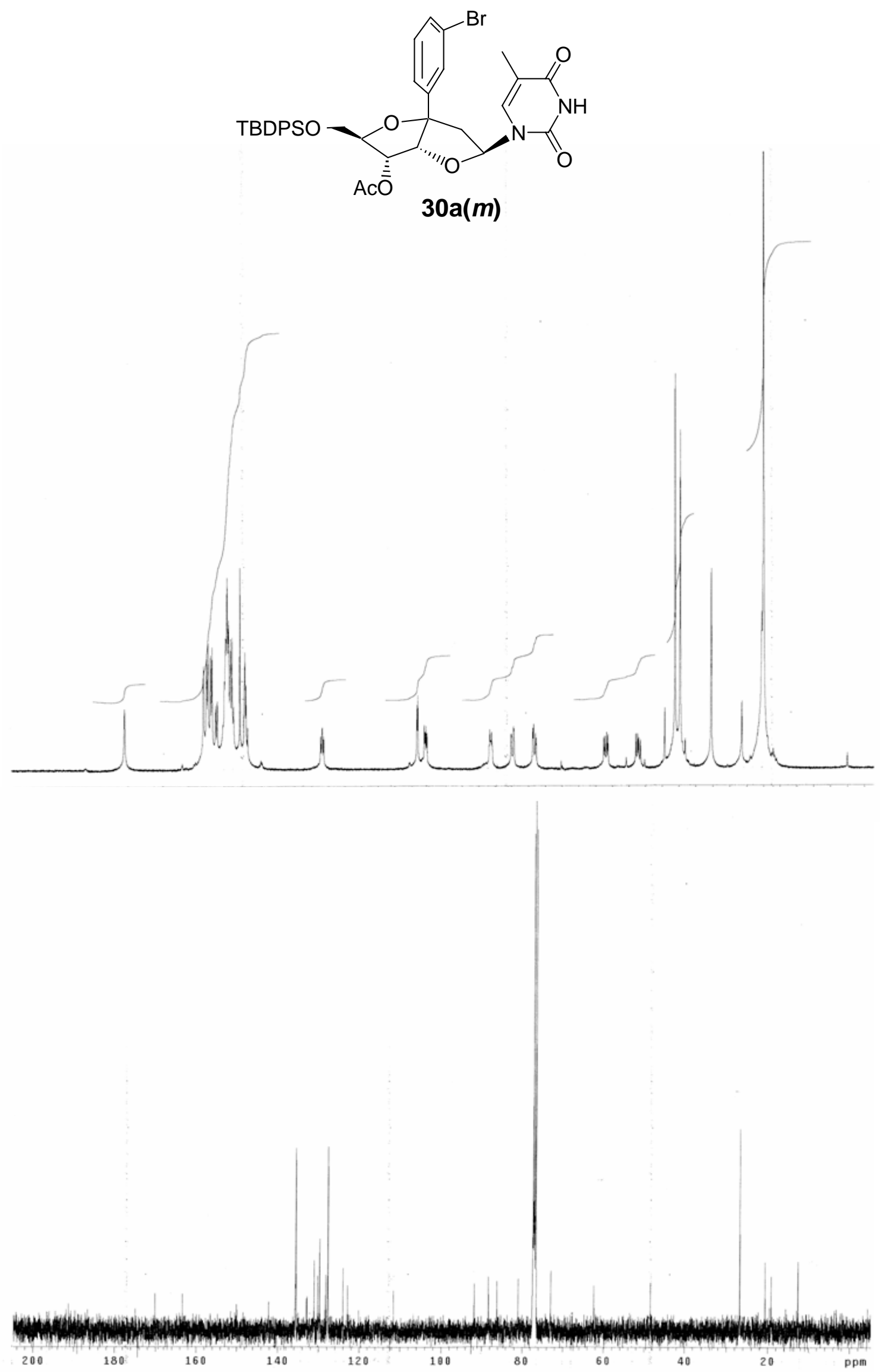

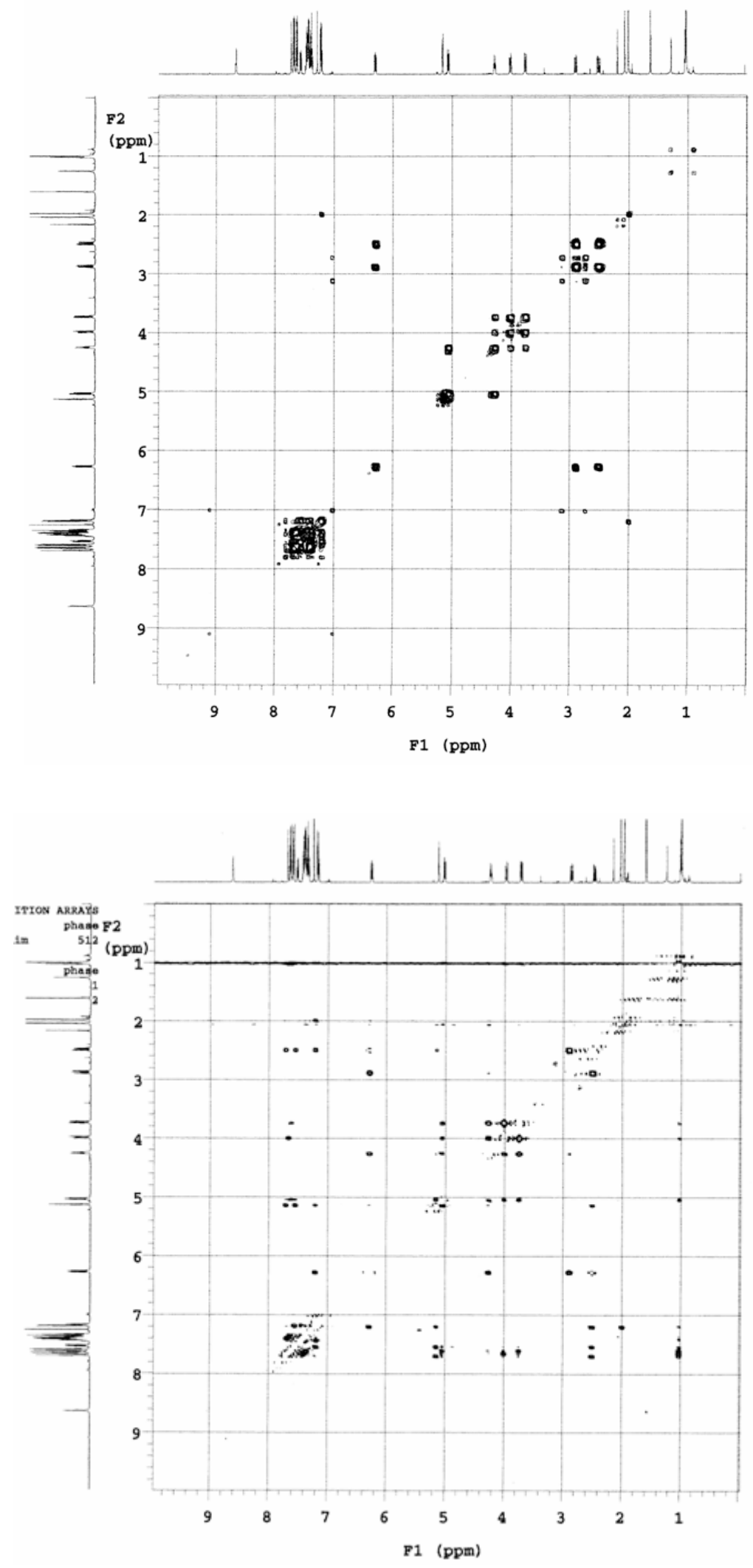

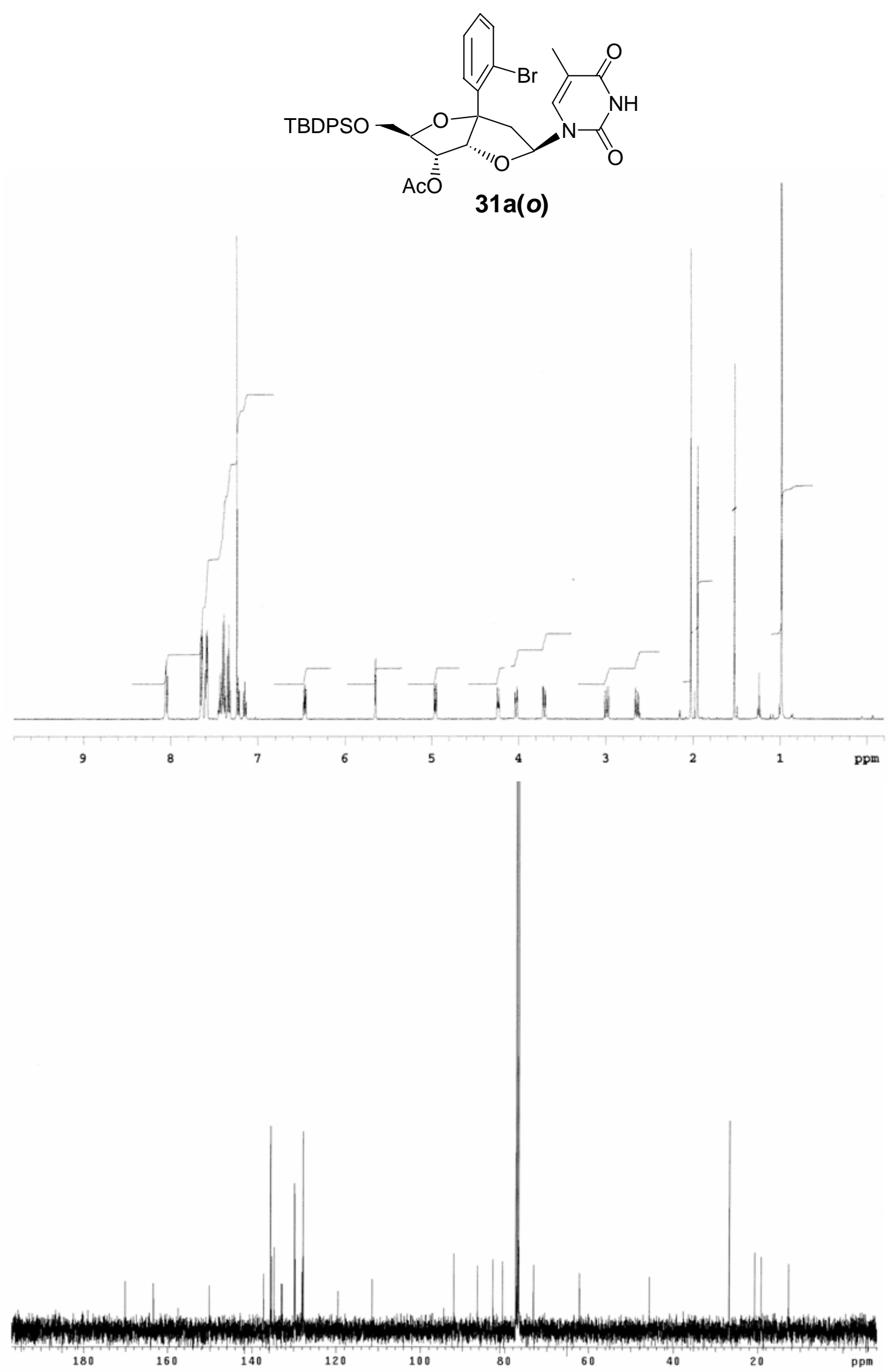

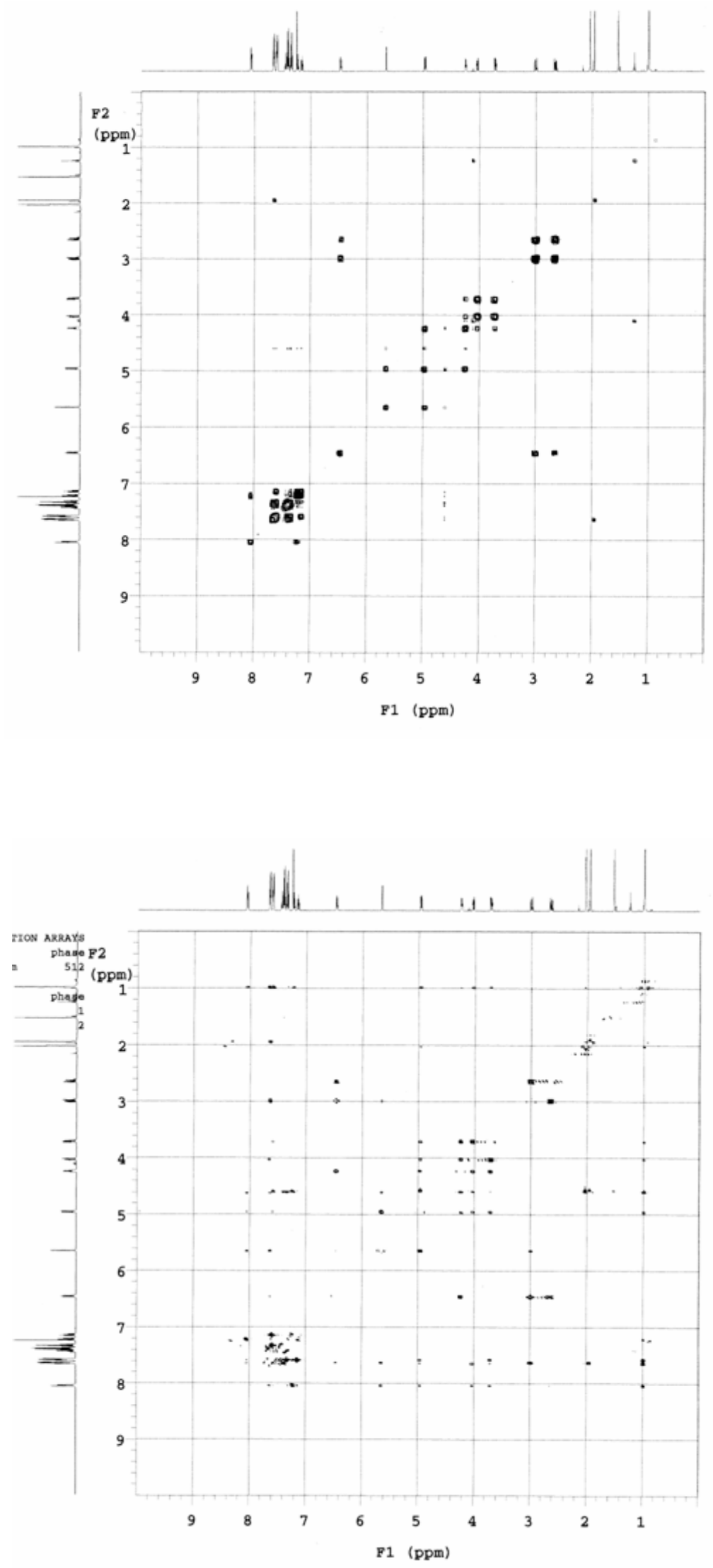

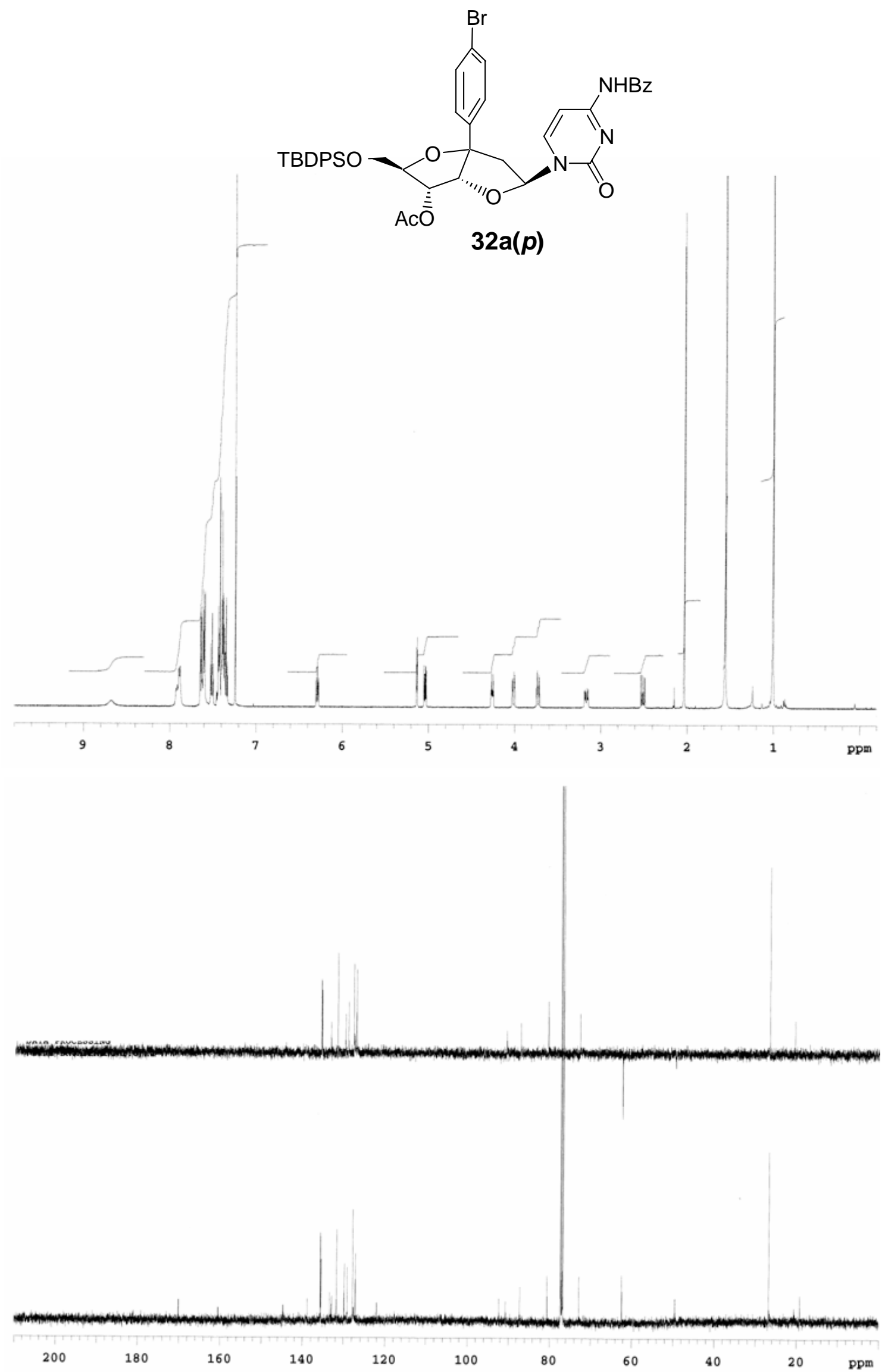

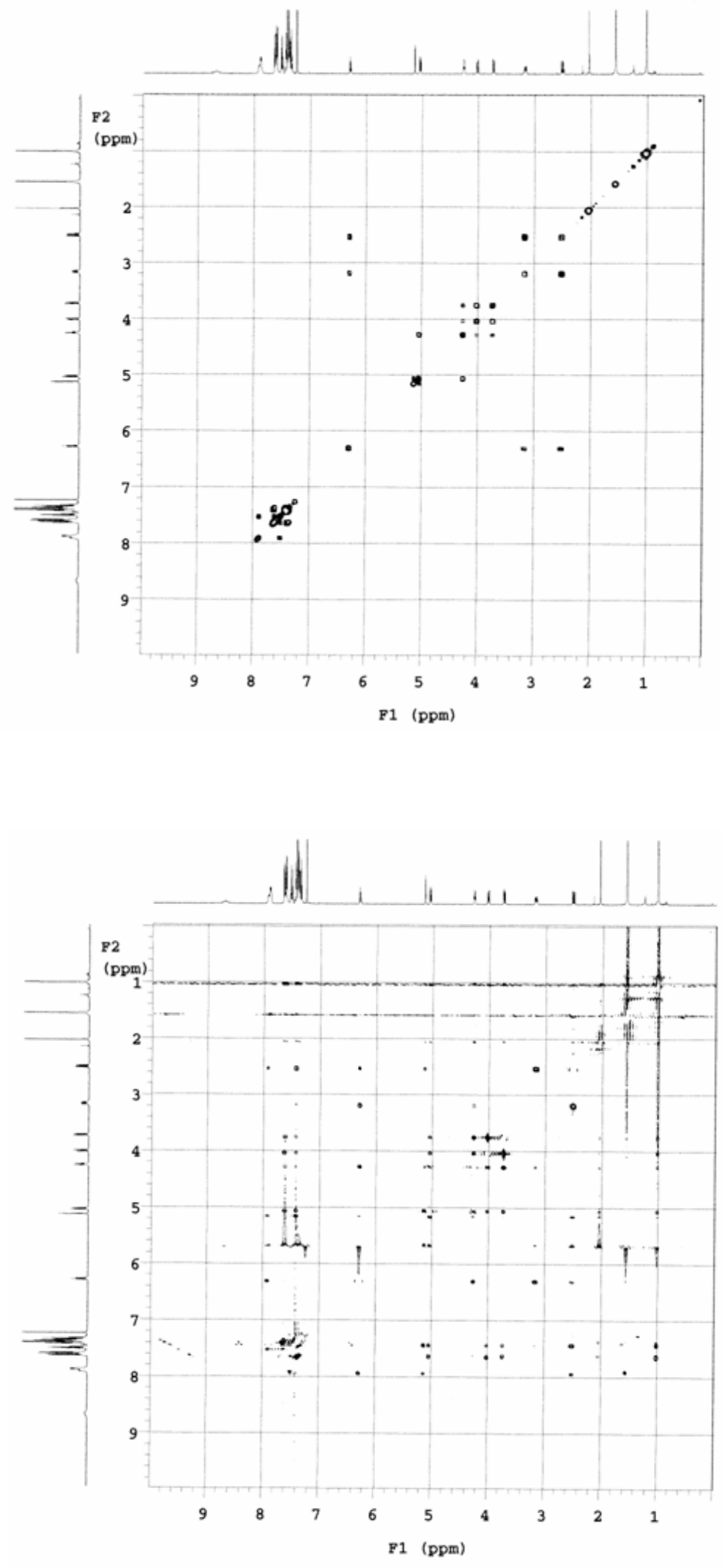

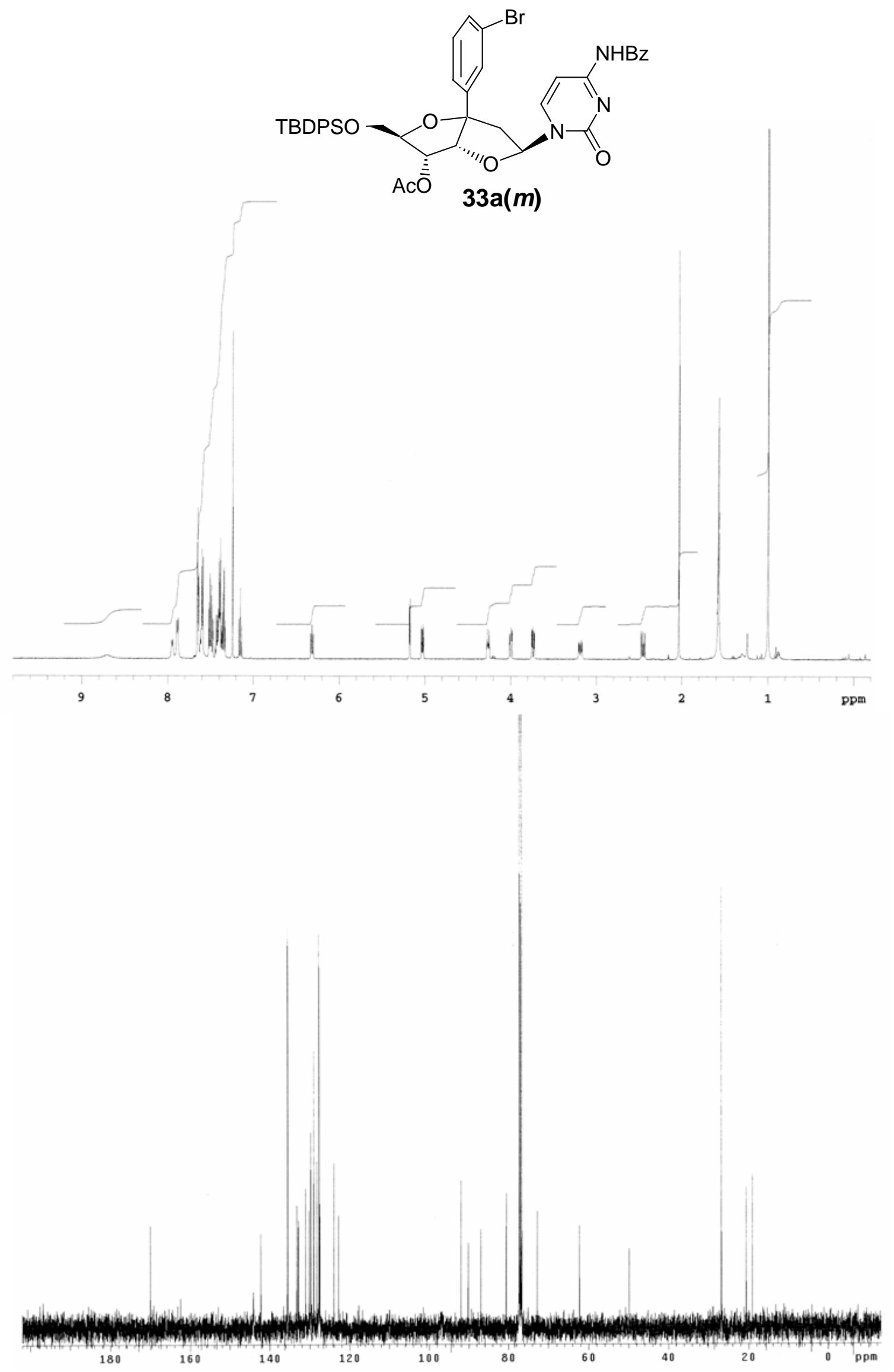

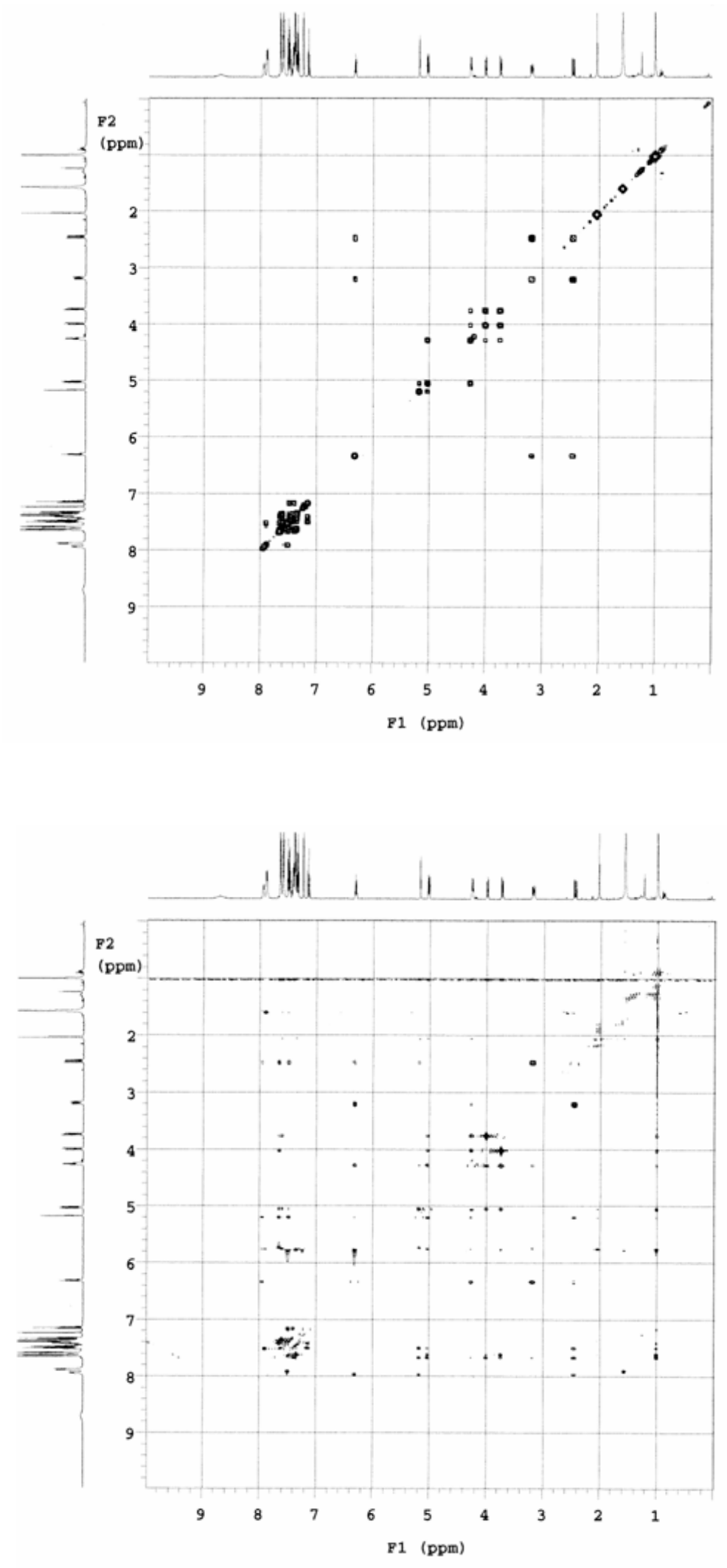

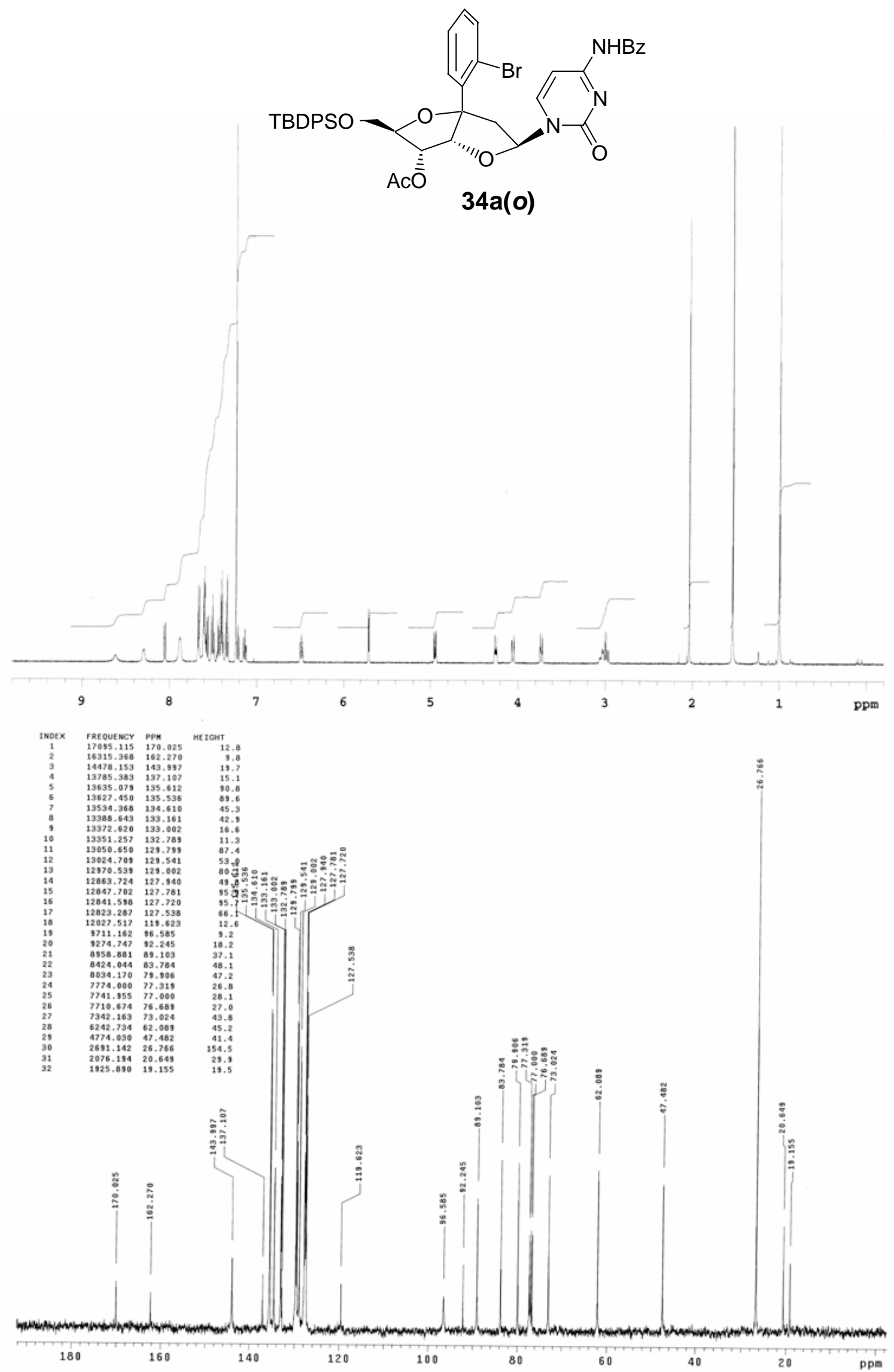

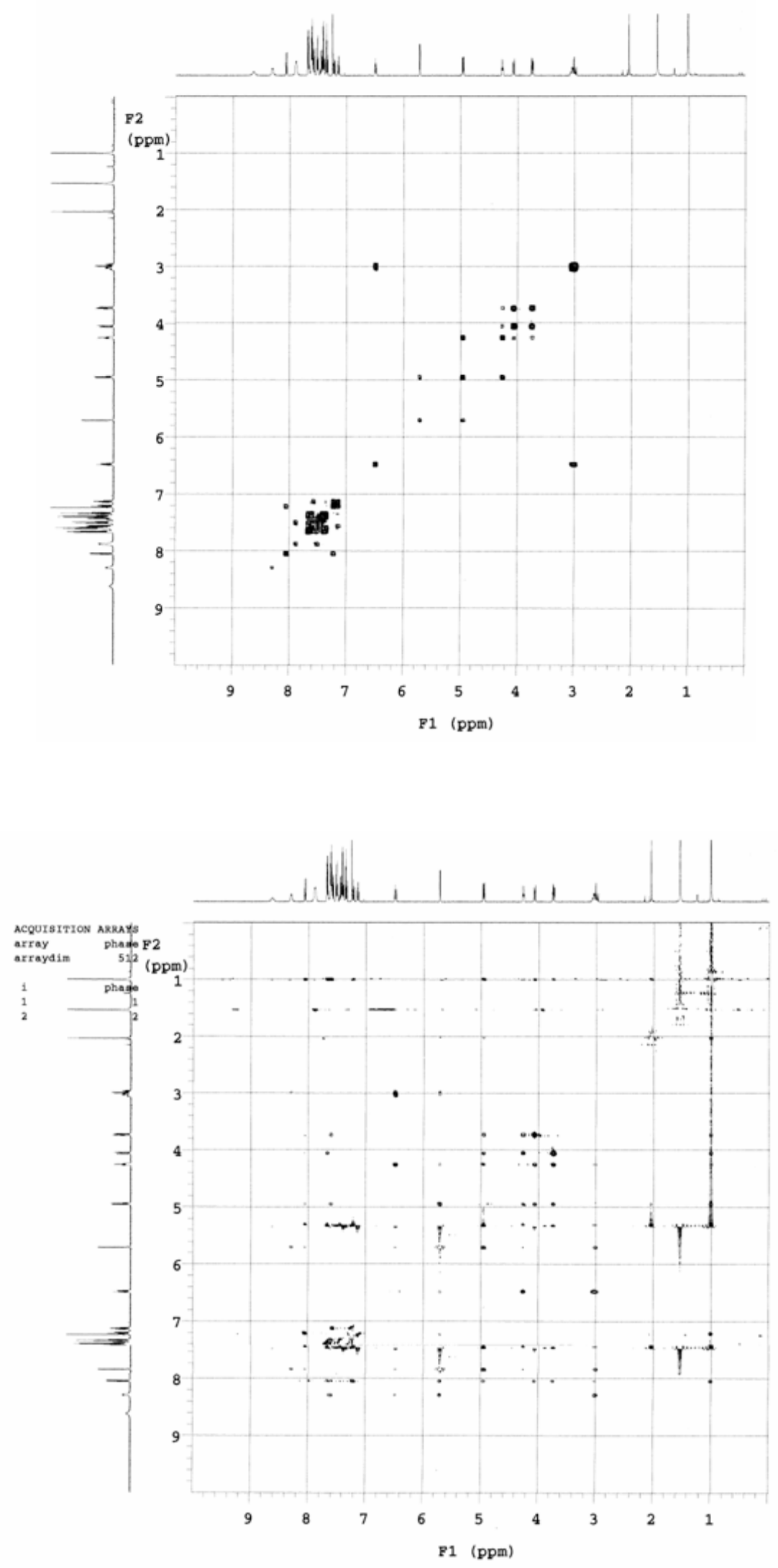


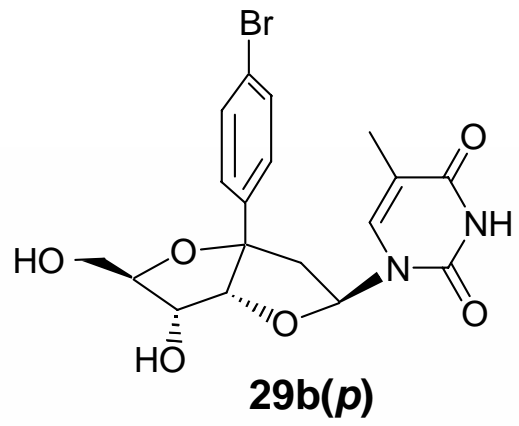
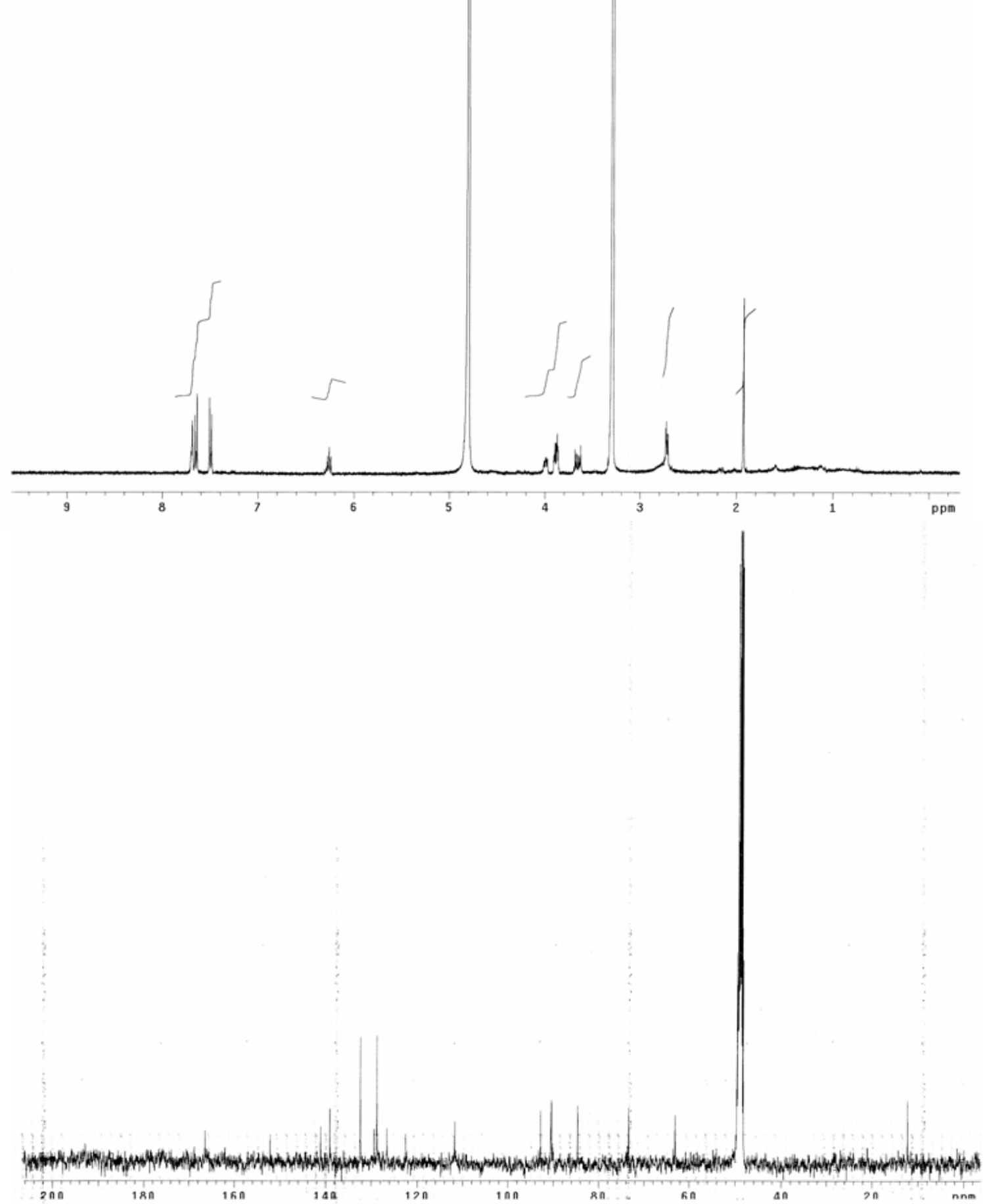
<smiles>Cc1cn([C@H]2C[C@H](O[Sb](=O)(=O)O)[C@@H](O)[C@@H](O)[C@H]2O)c(=O)[nH]c1=O</smiles>
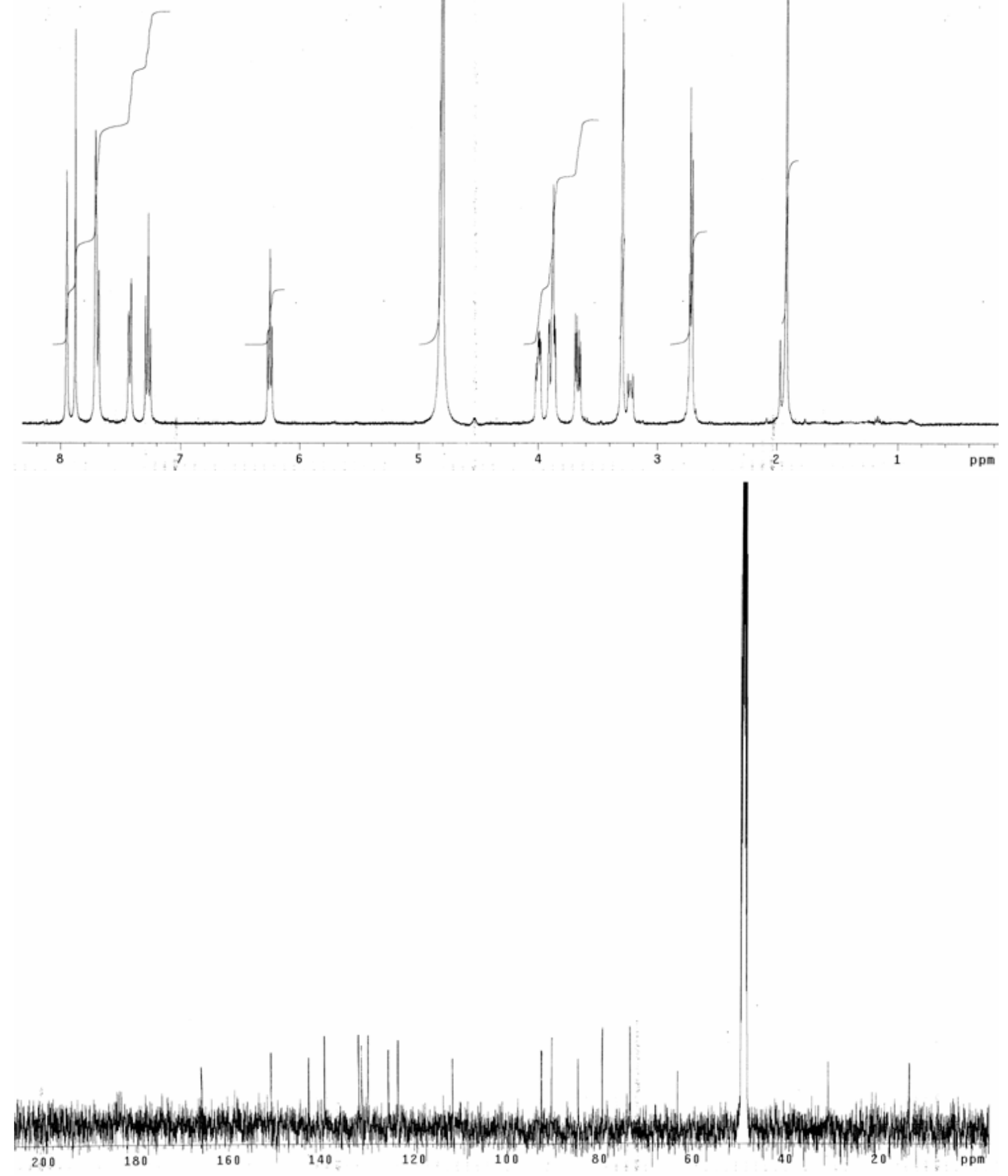

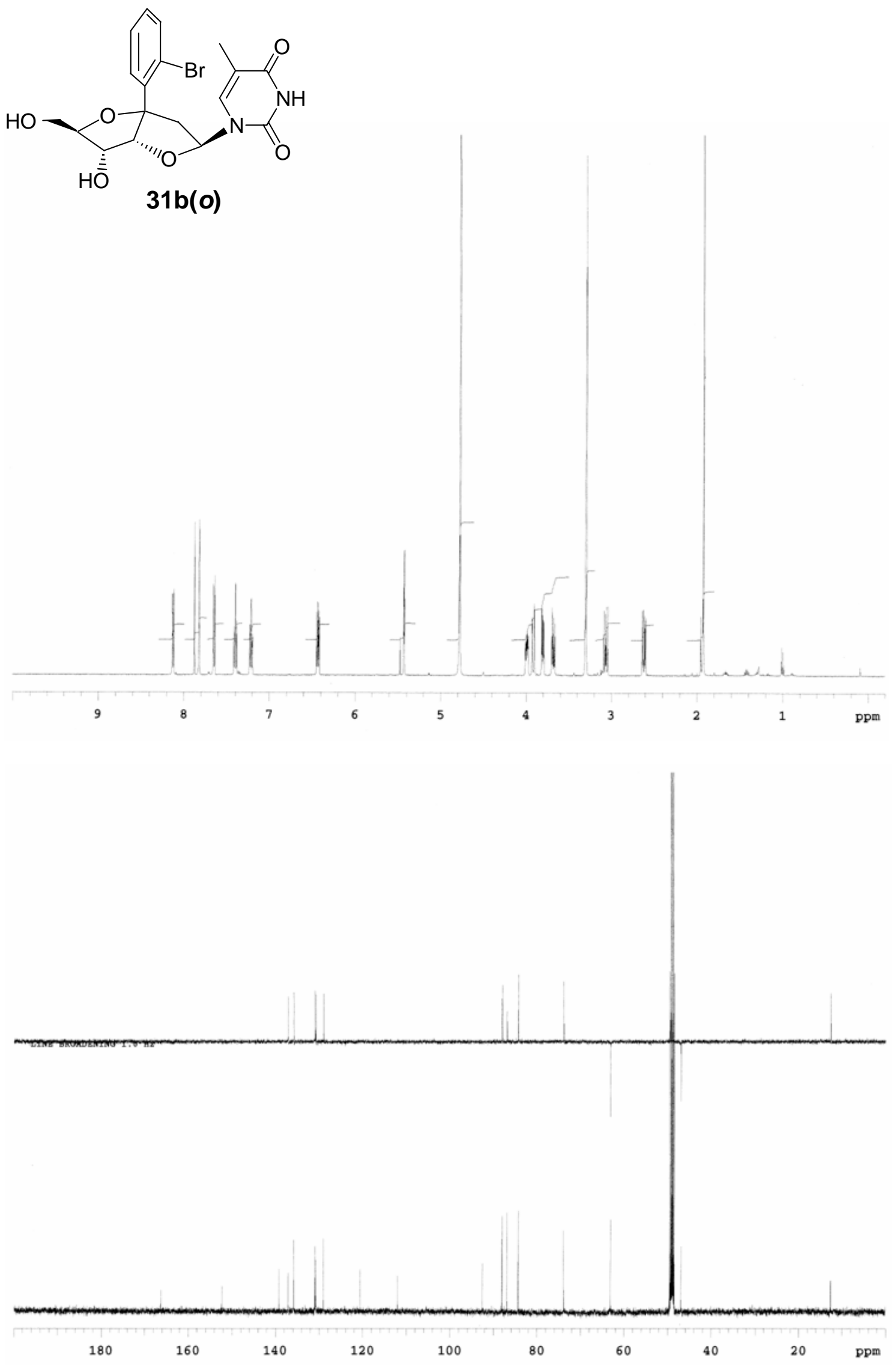

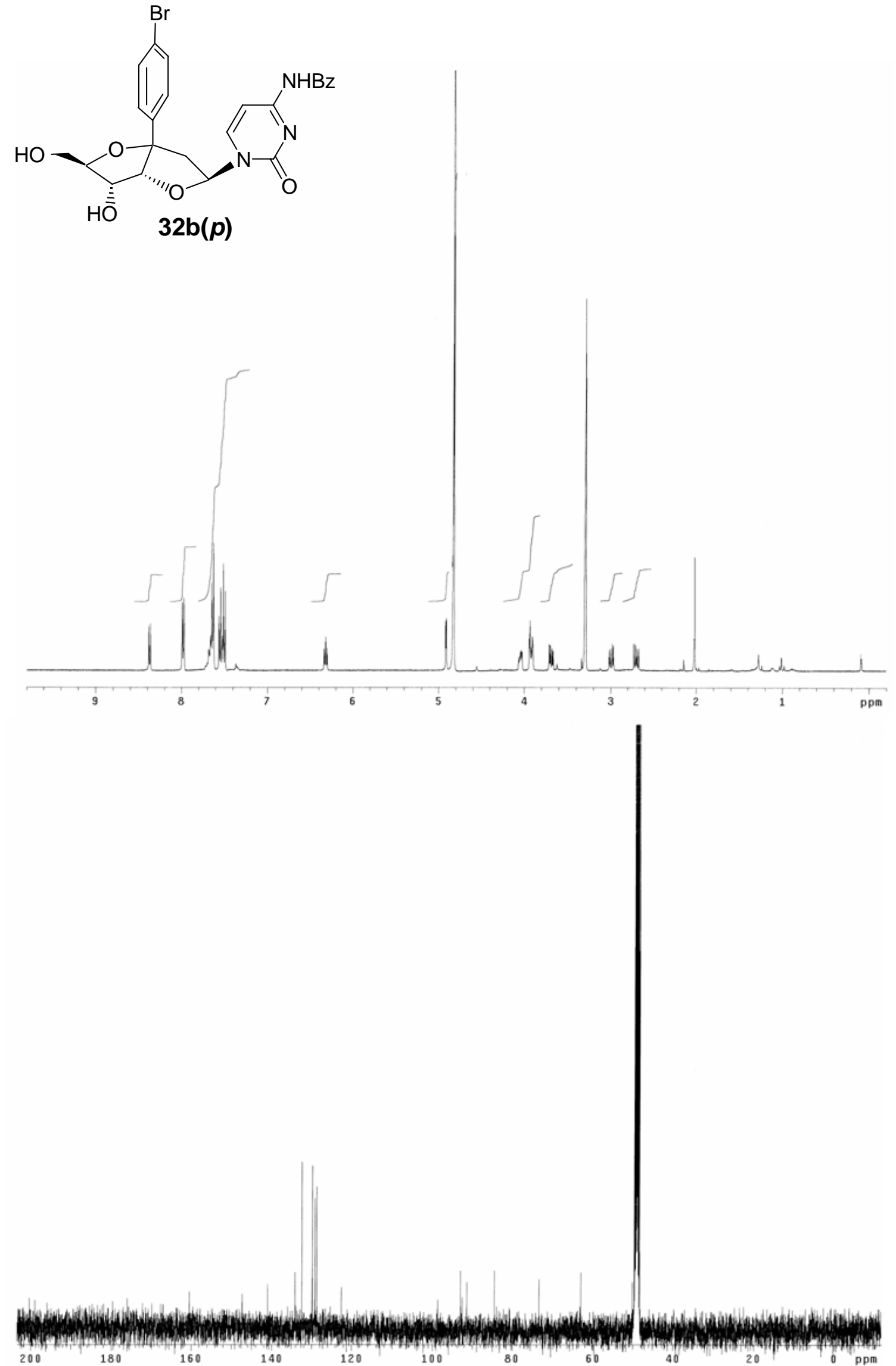


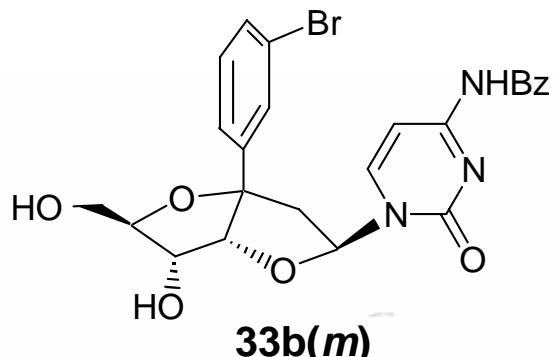

$33 b(m)$

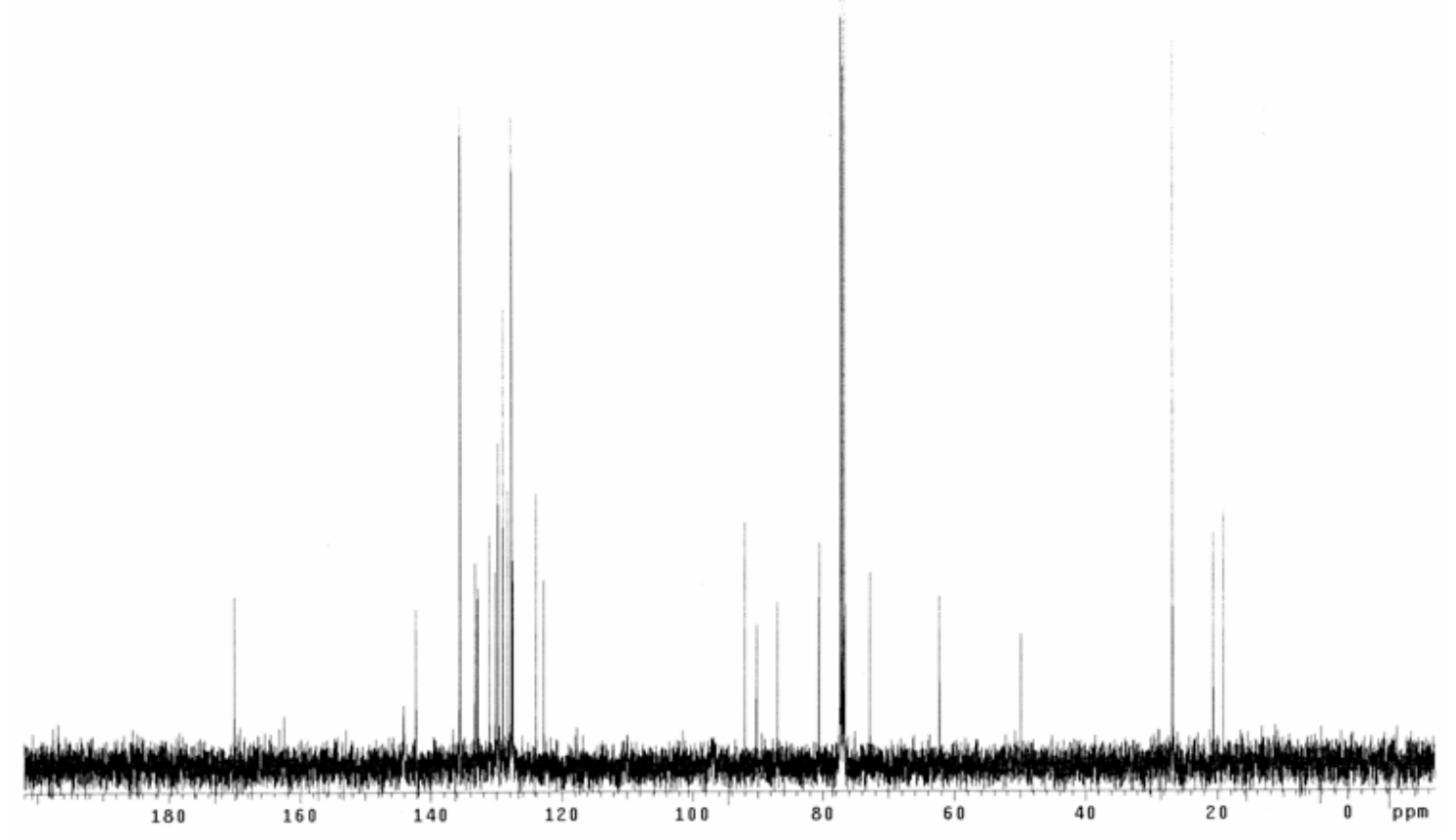



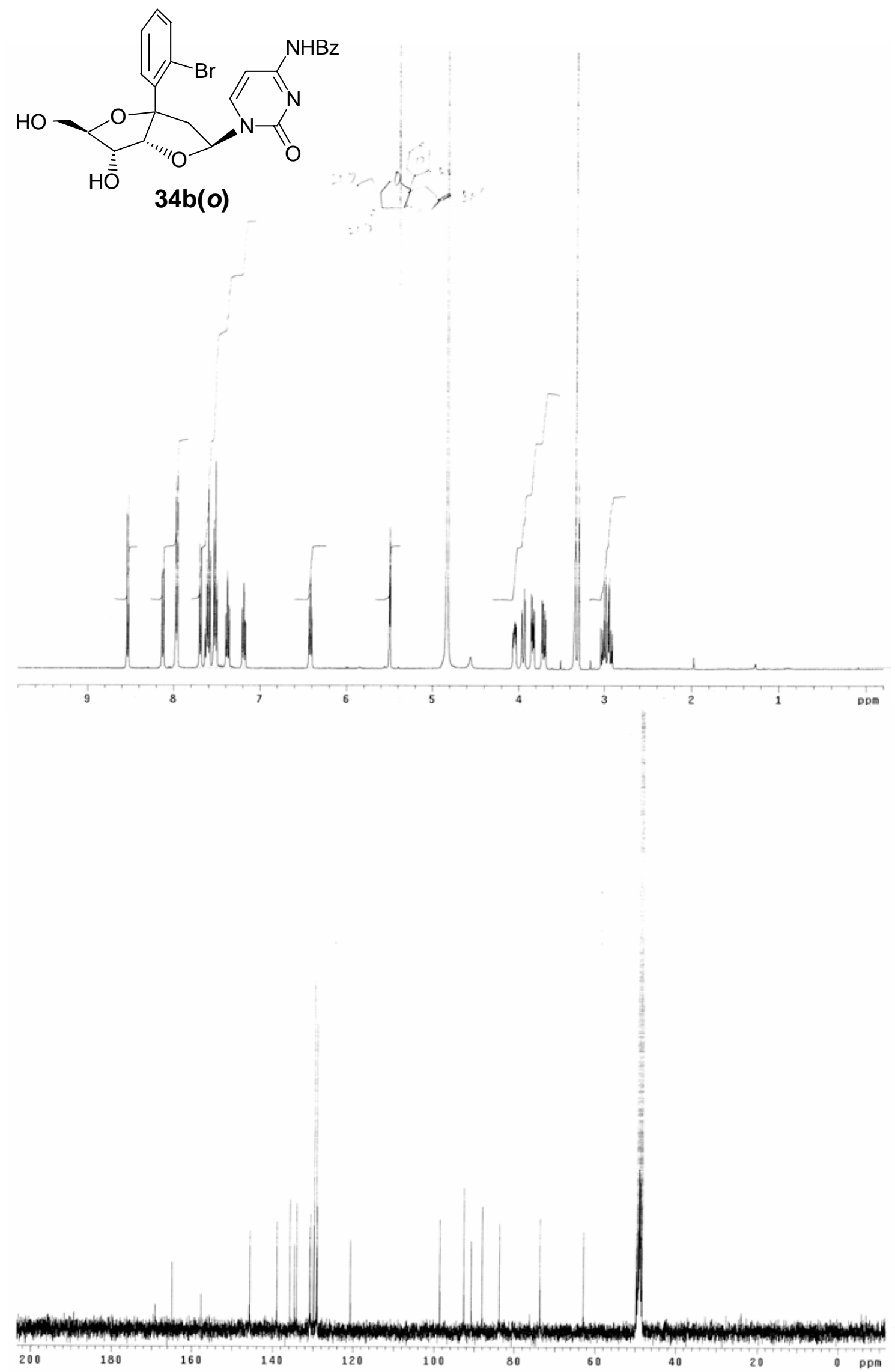

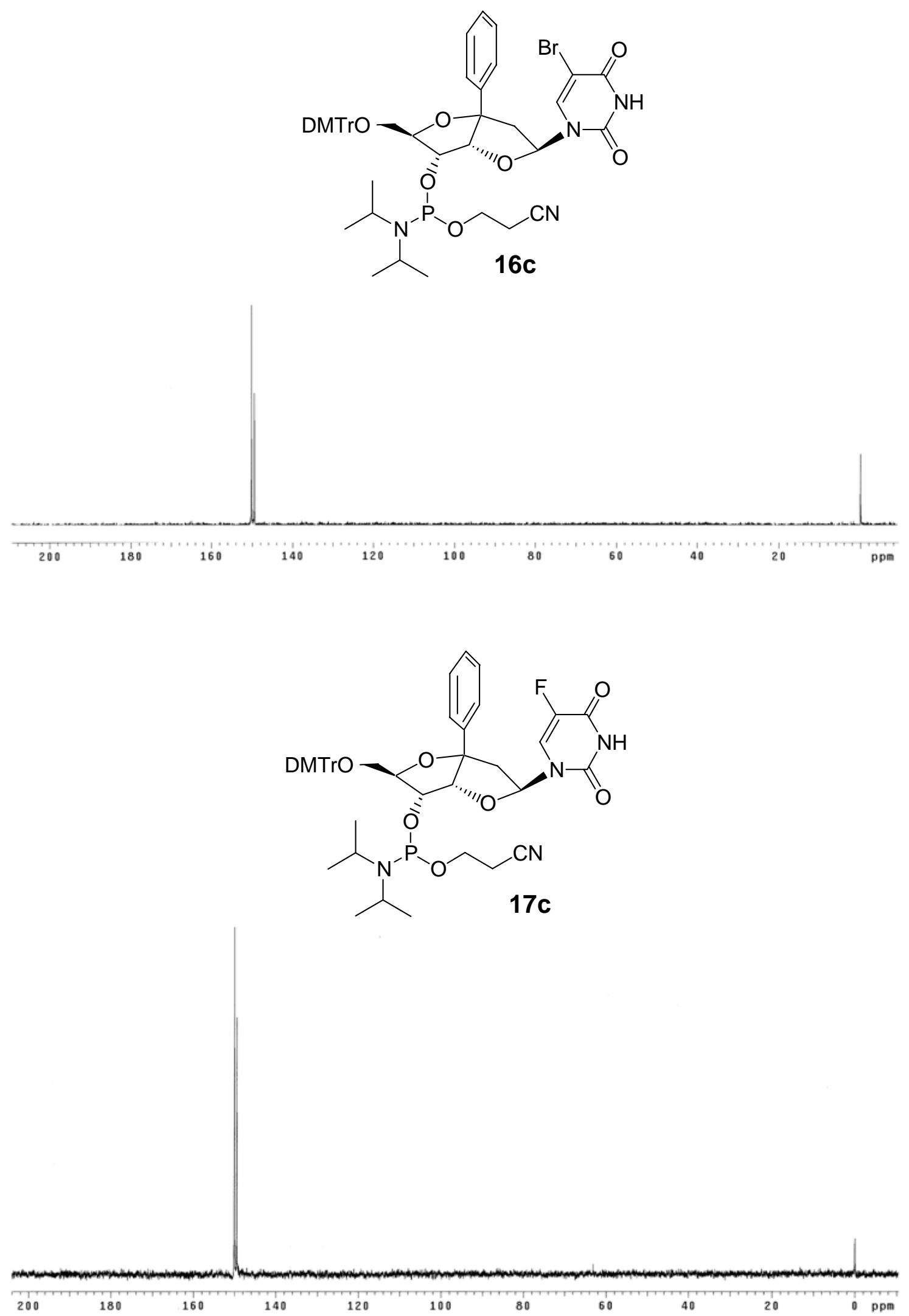

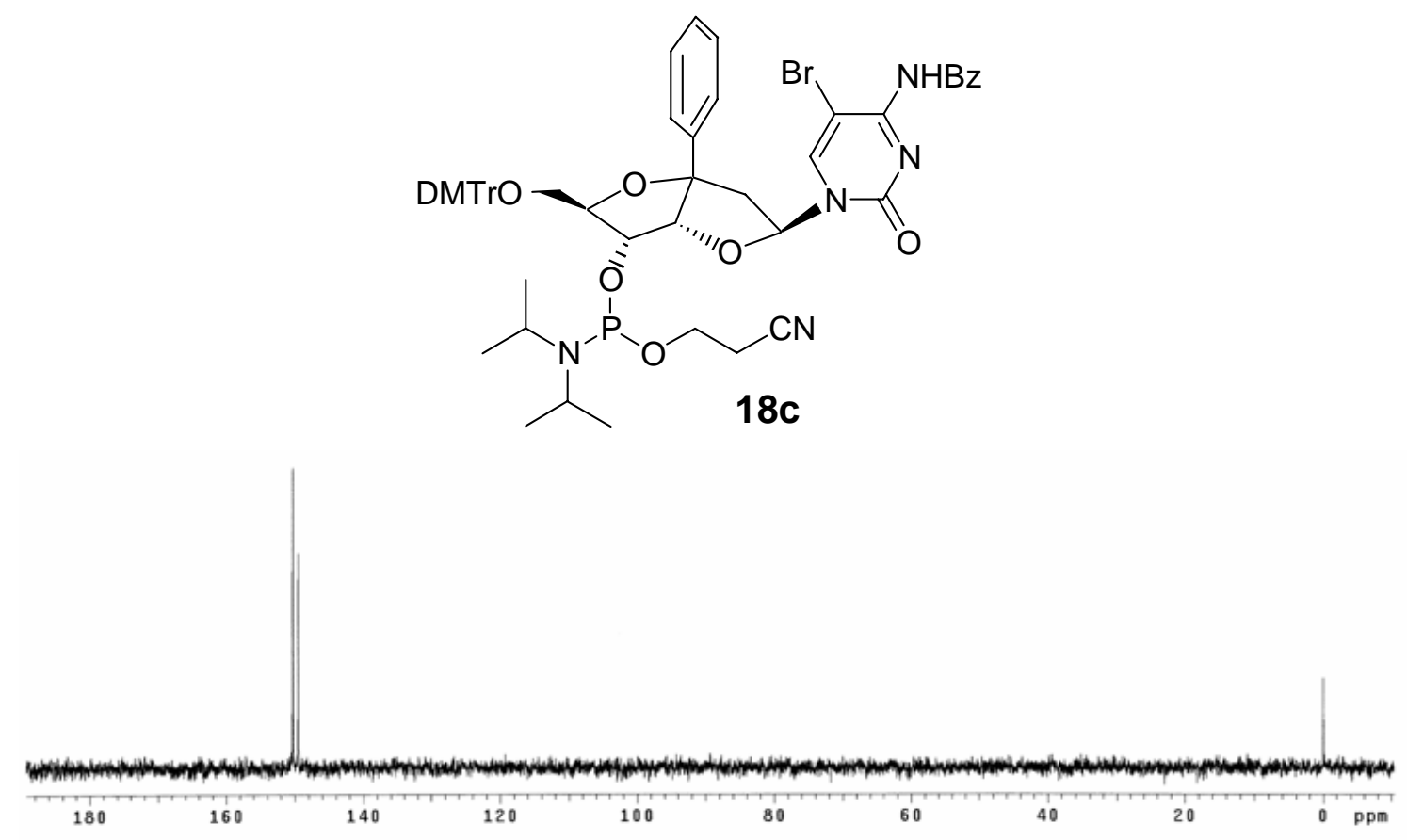

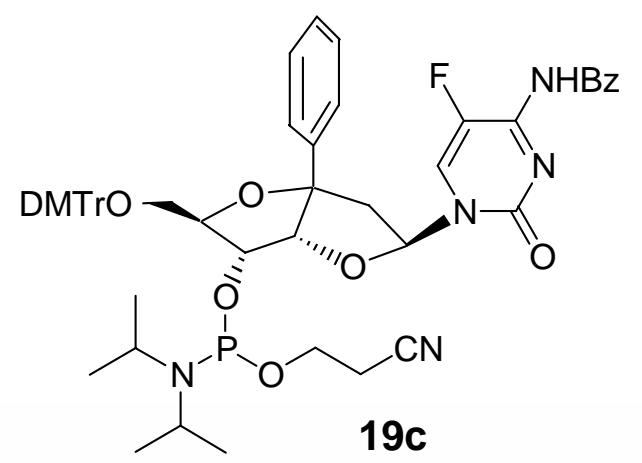

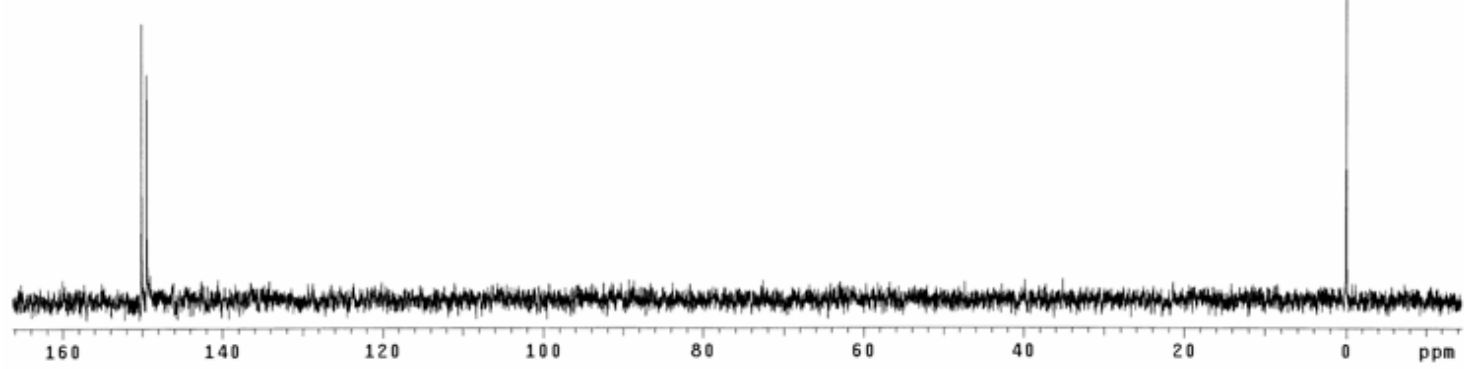



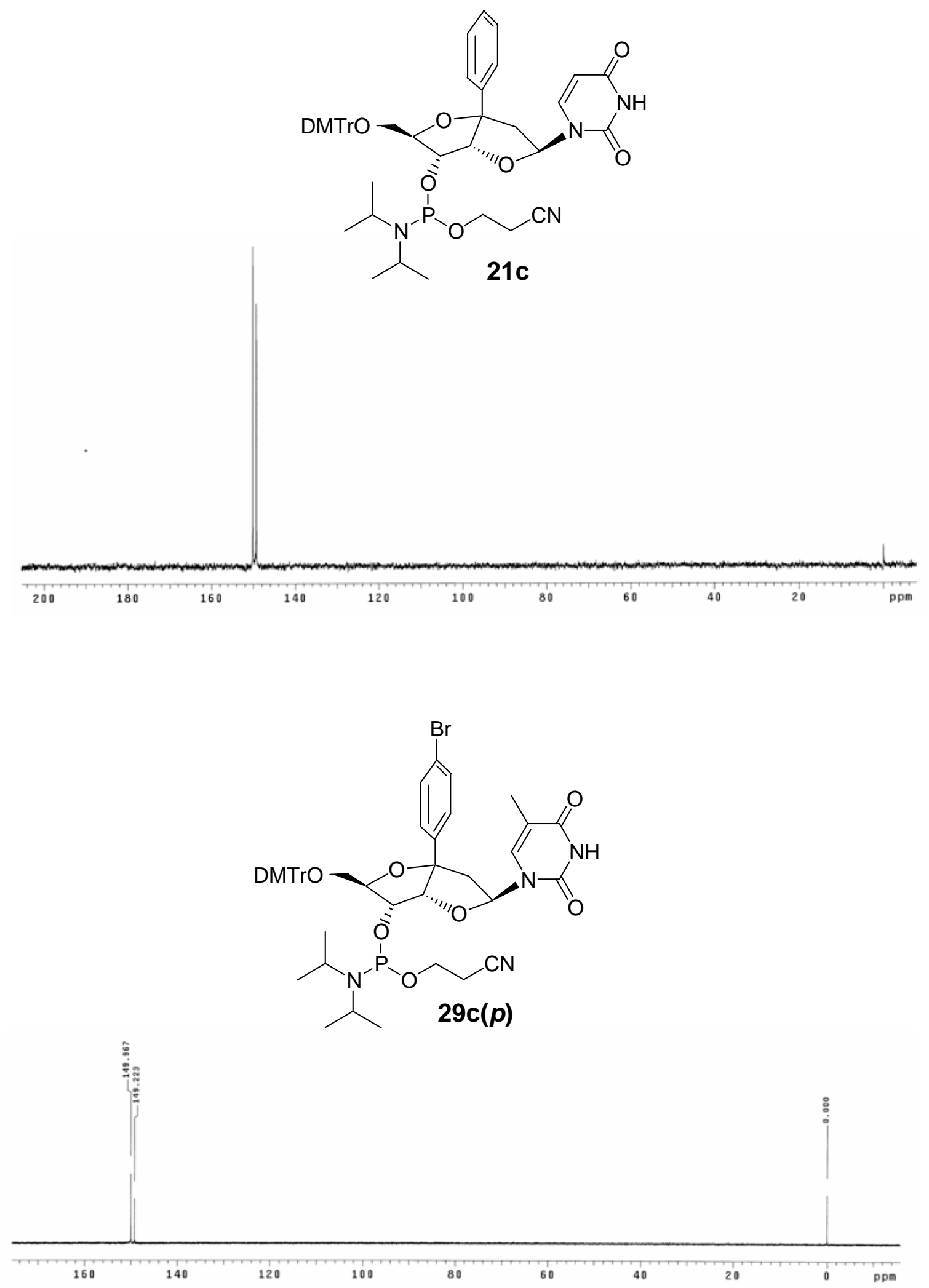

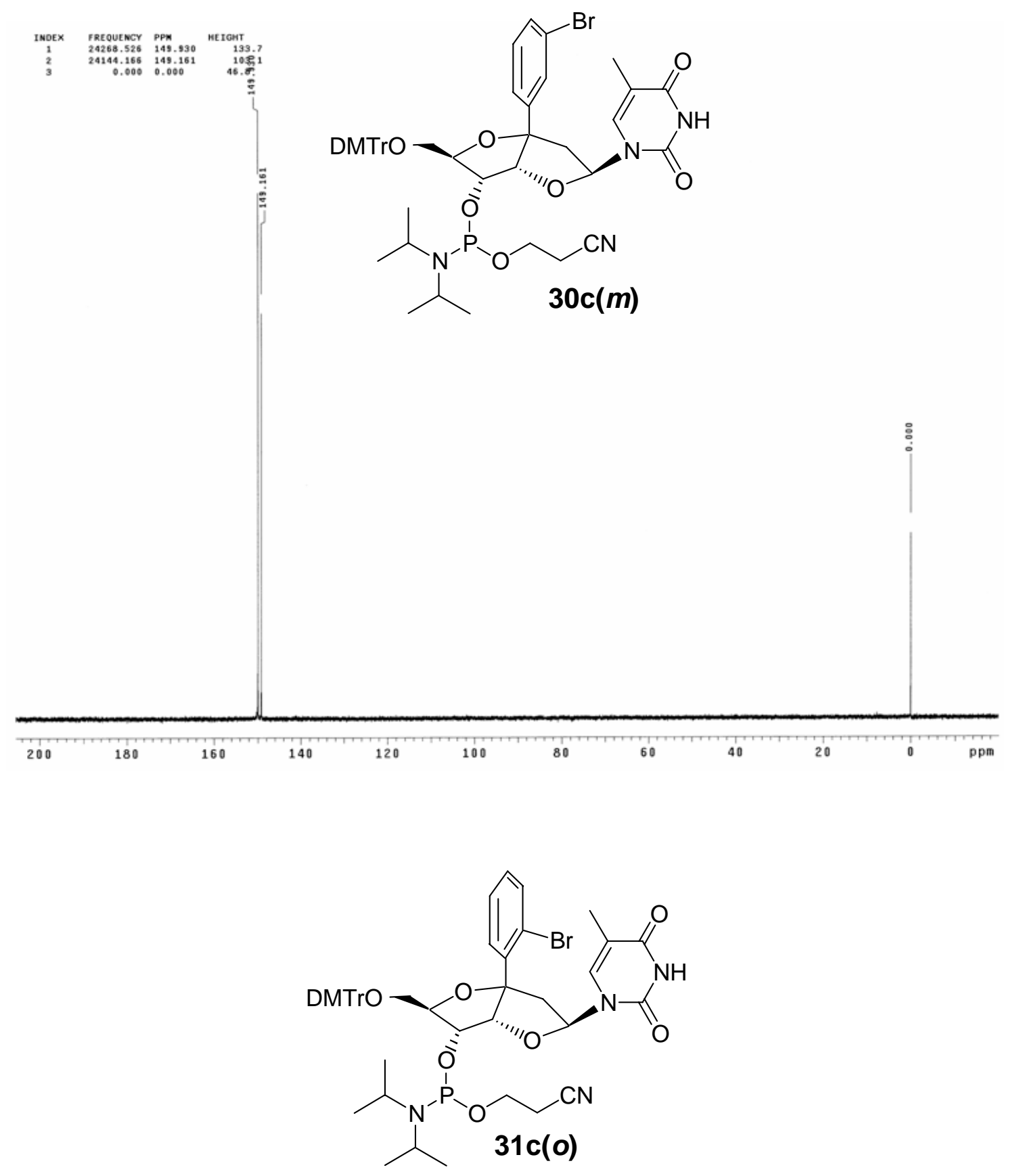

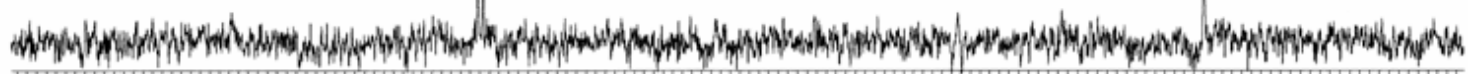
$\begin{array}{lllllllllllllll}240 & 220 & 200 & 180 & 160 & 140 & 120 & 100 & 80 & 60 & 40 & 20 & 0 & -20 & \mathrm{ppm}\end{array}$ 


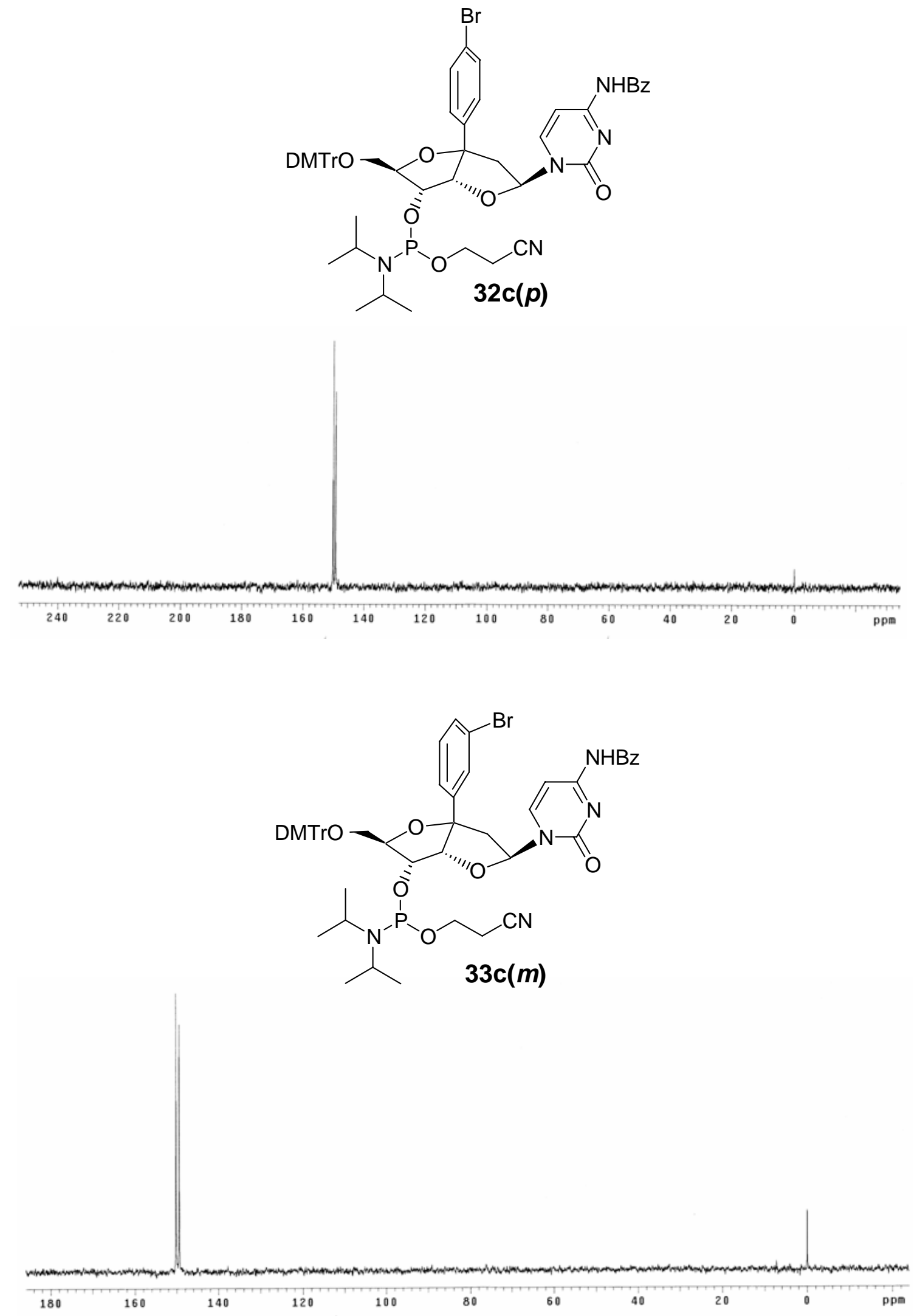



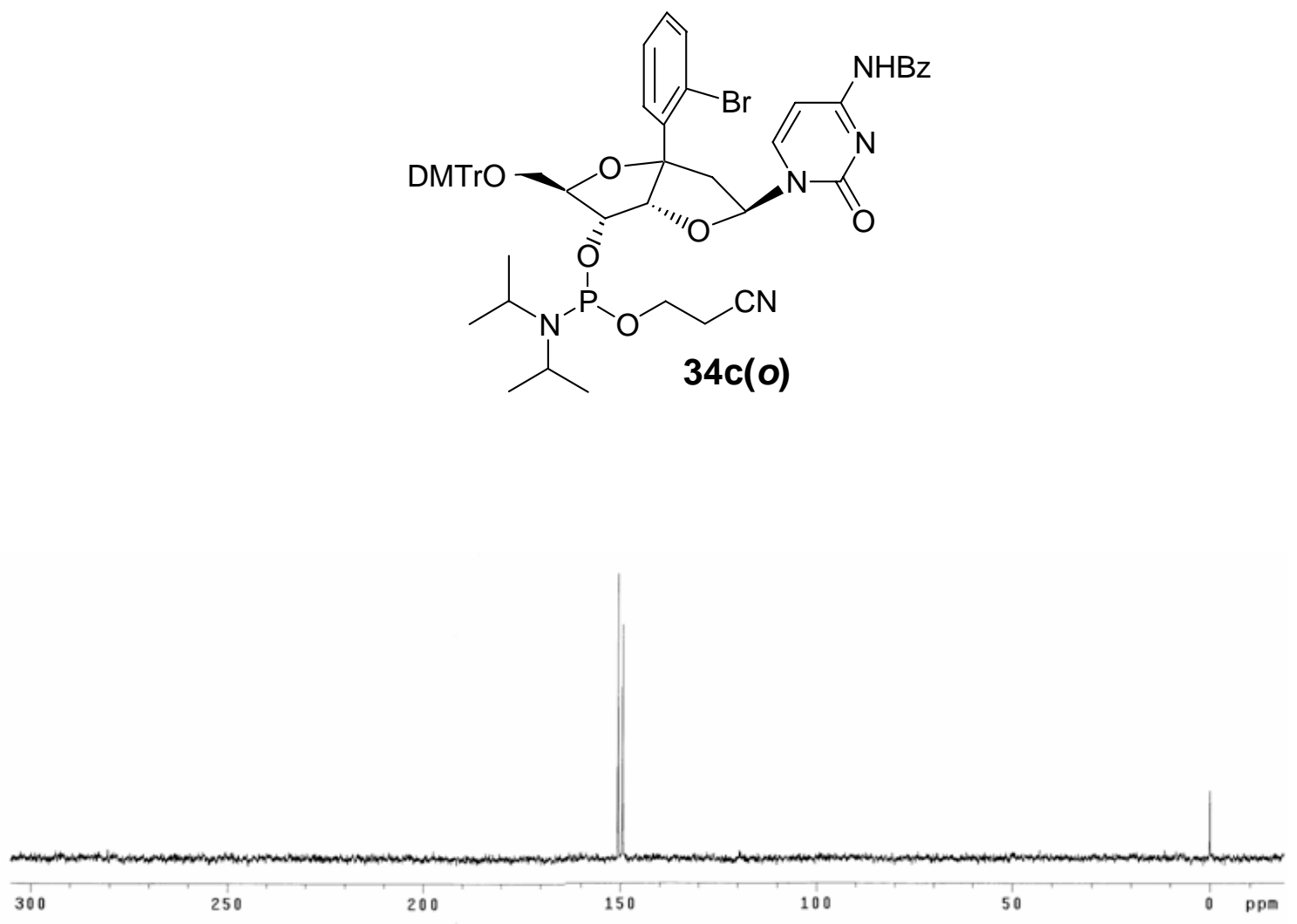